\title{
STOCHASTIC CONTROL OF TIDAL DYNAMICS EQUATION WITH LÉVY NOISE
}

\author{
POOJA AGARWAL, UTPAL MANNA, AND DEBOPRIYA MUKHERJEE
}

\begin{abstract}
In this work we first present the existence, uniqueness and regularity of the strong solution of the tidal dynamics model perturbed by Lévy noise. Monotonicity arguments have been exploited in the proofs. We then formulate a martingale problem of Stroock and Varadhan associated to an initial value control problem and establish existence of optimal controls.
\end{abstract}

\section{Contents}

1. Introduction

2. Tidal Dynamics: The Deterministic Model

3. Functional Setting

3.1. Preliminaries on Stochastic Processes

3.2. Stochastic Tide Model

4. Energy Estimates and Existence Result

4.1. $p^{\text {th }}$ Moment Estimate

4.2. Existence Result

5. Stochastic Optimal Control

5.1. Preliminaries

5.2. Martingale Problem

5.3. Existence of Martingale Solution

5.4. Existence of Optimal Control

Appendix A.

References

\section{INTRODUCTION}

Ocean-tide information has considerably many applications. The data obtained is used to solve vital problems in oceanography and geophysics, and to study earth tides, elastic properties of the Earth's crust and tidal gravity variations. It is also used in space studies to calculate the trajectories of man-made satellites of the Earth and to interpret the results of satellite measurements. The interaction of tides with deep sea ridges and chains of seamounts give rise to deep eddies which transport nutrients from the deep to the surface. The alternate flooding and exposure of the shoreline due to tides is an important factor in the determination of the ecology of the region.

One of the first mathematical explanation for tides was given by Newton by describing tide generating forces. The first dynamic theory of tides was proposed by Laplace. Here we consider the tidal dynamics model proposed by Marchuk and Kagan [32. The existence and uniqueness of weak solutions of the deterministic tide equation and that of strong solutions of the stochastic tide equation with additive trace class Gaussian noise have been proved in Manna, Menaldi and Sritharan 31]. In this work, we consider the stochastic tide equation with Lévy noise and prove the existence and uniqueness and regularity of solution in bounded domains. Control of fluid flow has numerous applications in control of pollutant transport, oil recovery/transport problems,

2010 Mathematics Subject Classification. 35Q35, 60H15, 76D03, 76D55.

Key words and phrases. Stochastic control, Initial value control, Tide equation, Minty-Browder theory, Martingale solution. 
weather predictions, control of underwater vehicles etc. Unification of many control problems in the engineering sciences have been done by studying the optimal control problem of Navier-Stokes equations (see [44, 45]). Here we consider the initial data optimal control of the stochastic tidal dynamics model. We consider the Stroock-Varadhan martingale formulation [46] of the stochastic model to prove the existence of optimal initial value control.

Organization of the paper is as follows. A brief description of the model has been given in Section 2 . Section 3 describes the functional setting of the problem and states the monotonicity property of the linear and non-linear operators. In Section 4 we consider the a-priori estimates and prove the existence, uniqueness and regularity of strong solution. In Section 5 we consider the stochastic optimal control problem with initial value control.

Let $\mathcal{O}$ be a bounded domain in $\mathbb{R}^{2}$ with smooth boundary. Let $\left(\Omega, \mathcal{F}, \mathcal{F}_{t}, P\right)$ be a given filtered probability space. In the framework of Gelfand triple $\mathbb{H}_{0}^{1}(\mathcal{O}) \subset \mathbb{L}^{2}(\mathcal{O}) \subset \mathbb{H}^{-1}(\mathcal{O})$, we consider the following tidal dynamics model with Lévy noise

$$
\begin{aligned}
& d u(t)+[A u(t)+B(u(t))+g \nabla \hat{z}(t)] d t=f(t) d t+\sigma(t, u(t)) d W(t) \\
& +\int_{Z} H(u(t-), z) \tilde{N}(d t, d z), \quad \text { in } \quad[0, T] \times \mathcal{O} \times \Omega, \\
& d \hat{z}(t)+\operatorname{Div}(h u(t)) d t=0 \quad \text { in } \quad[0, T] \times \mathcal{O} \times \Omega, \\
& u(0, x, \omega)=u_{0}(x, \omega), \quad \hat{z}(0, x, \omega)=\hat{z}_{0}(x, \omega),(x, \omega) \in \mathcal{O} \times \Omega .
\end{aligned}
$$

The operators $A$ and $B$ are defined in Sections 2 and $3 .(W(t))_{t \geq 0}$ is an $\mathbb{L}^{2}$-valued Wiener process with trace class covariance. $\tilde{N}(d t, d z)=N(d t, d z)-\lambda(d z) d t$ is a compensated Poisson random measure, where $N(d t, d z)$ denotes the Poisson counting measure associated to the point process $p(t)$ on $Z \in \mathscr{B}\left(\mathbb{L}^{2} \backslash\{0\}\right)$, where the solutions of the above system have its paths, and $\lambda(d z)$ is a $\sigma$-finite measure on $(Z, \mathscr{B}(Z))$.

The following theorem states the main result of Section 4 . The functional spaces appearing in the statement of the theorem have been defined in Section 3 .

Theorem 1.1. Let us consider the above stochastic tide model with $f, u_{0}$ and $\hat{z}_{0}$ such that

$$
f \in L^{2}\left(\Omega ; L^{2}\left(0, T ; \mathbb{L}^{2}(\mathcal{O})\right)\right), \quad u_{0} \in L^{2}\left(\Omega ; \mathbb{L}^{2}(\mathcal{O})\right), \quad \hat{z}_{0} \in L^{2}\left(\Omega ; L^{2}(\mathcal{O})\right) .
$$

Let the noise coefficients $\sigma$ and $H$ satisfy Assumption 3.12. Then there exist path-wise unique adapted processes $u(t, x, \omega)$ and $\hat{z}(t, x, \omega)$ with the regularity

$$
\left\{\begin{array}{l}
u \in L^{2}\left(\Omega ; L^{\infty}\left(0, T ; \mathbb{L}^{2}(\mathcal{O})\right) \cap L^{2}\left(0, T ; \mathbb{H}_{0}^{1}(\mathcal{O})\right)\right) \\
\hat{z} \in L^{2}\left(\Omega ; C\left([0, T] ; L^{2}(\mathcal{O})\right)\right)
\end{array}\right.
$$

satisfying the the stochastic tide model (1.1)-(1.3) in the weak sense.

In Section 5. we consider the following stochastic optimal control problem with initial value control,

$$
\begin{aligned}
d u(t)+ & {[A u(t)+B(u(t))+g \nabla \hat{z}(t)] d t=f(t) d t+\sigma(t, u(t)) d W(t) } \\
& +\int_{Z} H(u(t-), z) \tilde{N}(d t, d z) \quad \text { in }[0, T] \times \mathcal{O} \times \Omega, \\
d \hat{z}(t)+ & D i v(h u(t)) d t=0 \quad \text { in }[0, T] \times \mathcal{O} \times \Omega, \\
u(0)= & u_{0}+U, \quad \hat{z}(0)=\hat{z}_{0},
\end{aligned}
$$

where $u_{0} \in L^{2}\left(\Omega ; \mathbb{L}^{2}(\mathcal{O})\right), \hat{z}_{0} \in L^{2}\left(\Omega ; L^{2}(\mathcal{O})\right)$ and $U \in L^{2}\left(\Omega ; \mathbb{L}^{2}(\mathcal{O})\right)$. The regularities on the initial values and the assumptions on $\sigma$ and $H$ are the same as considered in Theorem 1.1 The cost functional is given by

$$
\mathcal{J}(u, \hat{z}, U)=\mathbb{E}\left[\int_{0}^{T} \int_{\mathcal{O}} L(t, u, \hat{z}, U) d x d t\right],
$$

where the function $L$ is defined in Section 5

The main result of Section [5] is the following: 
Theorem 1.2. Suppose there exists $u_{0} \in L^{2}\left(\Omega ; \mathbb{L}^{2}(\mathcal{O})\right)$ and $\hat{z}_{0} \in L^{2}\left(\Omega ; L^{2}(\mathcal{O})\right)$ and $\pi \in \overline{\mathcal{U}}_{\text {ad }}^{w}\left(u_{0}, \hat{z}_{0}, T\right)$ such that $J(\pi)<+\infty$, where $J$ is defined by (5.80). Then the optimal control problem admits a weak optimal control with time horizon $[0, T]$ where $\overline{\mathcal{U}}_{a d}^{w}\left(u_{0}, \hat{z}_{0}, T\right)$ denotes the set of all weak admissible controls (with time horizon $[0, T]$ ).

\section{Tidal Dynamics: The Deterministic Model}

Under the assumptions that: (1) Earth is perfectly solid, (2) ocean tides do not change Earth's gravitational field, and (3) no energy exchange takes place between the mid-ocean and shelf zone, Marchuk and Kagan [32] obtained the following mathematical model

$$
\begin{gathered}
\partial_{t} w+A_{1} w-\kappa_{h} \Delta w+\frac{r}{h}|w| w+g \nabla \xi=f, \\
\partial_{t} \xi+\operatorname{Div}(h w)=0,
\end{gathered}
$$

in $[0, T] \times \mathcal{O}$, where $\mathcal{O}$ is a bounded 2-D domain (horizontal ocean basin) with coordinates $x=$ $\left(x_{1}, x_{2}\right)$ and $t$ represents the time. Here $\partial_{t}$ denotes the time derivative, $\triangle, \nabla$ and Div are the Laplacian, gradient and the divergence operators respectively.

The unknown variables $(w, \xi)$ represent the total transport 2-D vector (i.e., the vertical integral of the velocity from the ocean surface to the ocean floor) and the displacement of the free surface with respect to the ocean floor. The coefficients $A_{1}=\left[a_{i j}\right]$ is a 2-dimensional antisymmetric square matrix with constant coefficients $a_{11}=a_{22}=0$ and $-a_{12}=a_{21}=2 \omega_{z}$, the Coriolis parameter (i.e., $\omega_{z}=\omega \cos (\varphi), \omega$ is the angular velocity of the Earth rotation and $\varphi$ the latitude), $\kappa_{h}>0$ the constant horizontal macro turbulent viscosity coefficient, $r>0$ the constant bottom friction coefficient equal to a numerical constant, $g$ the Earth gravitational constant, $h=h(x)$ is the (vertical) depth at $x$ in the region $\mathcal{O}$ and $f=\gamma_{L} g \nabla \xi^{+}$is the known tide-generating force with $\gamma_{L}$ the Love factor.

Following Manna et al. [31, and Marchuk et al. [32, we denote by $A$ the following matrix operator

$$
A:=\left(\begin{array}{cc}
-\alpha \triangle & -\beta \\
\beta & -\alpha \triangle
\end{array}\right),
$$

and the nonlinear vector operator

$$
v \mapsto \gamma|v| v:=\left(\begin{array}{c}
\gamma(x) v_{1} \sqrt{v_{1}^{2}+v_{2}^{2}} \\
\gamma(x) v_{2} \sqrt{v_{1}^{2}+v_{2}^{2}}
\end{array}\right),
$$

where $\alpha:=\kappa_{h}$ and $\beta:=2 \omega \cos (\varphi)$ are positive constants, $\gamma(x):=r / h(x)$ is a strictly positive smooth function. In this model we assume the depth $h(x)$ to be a continuously differentiable function of $x$, nowhere becoming zero, so that

$$
\min _{x \in \mathcal{O}} h(x)=\epsilon>0, \quad \max _{x \in \mathcal{O}} h(x)=\mu, \quad \max _{x \in \mathcal{O}}|\nabla h(x)| \leq M,
$$

where $\mathrm{M}$ is some positive constant which equals zero at a constant ocean depth.

To reduce to homogeneous Dirichlet boundary conditions consider the natural change of unknown functions

$$
u(t, x):=w(t, x)-w^{0}(t, x),
$$

and

$$
\hat{z}(t, x):=\xi(t, x)+\int_{0}^{t} \operatorname{Div}\left(h w^{0}(s, x)\right) d s,
$$

which are referred to the tidal flow and the elevation. The full flow $w^{0}$ which is given a-priori on the boundary $\partial \mathcal{O}$, has been extended to the whole domain $[0, T] \times \mathcal{O}$ as a smooth function and still denoted by $w^{0}$. 
Then the tidal dynamic equation can be written as

$$
\left\{\begin{array}{l}
\partial_{t} u+A u+\gamma\left|u+w^{0}\right|\left(u+w^{0}\right)+g \nabla \hat{z}=f^{\prime} \quad \text { in } \quad[0, T] \times \mathcal{O} \\
\partial_{t} \hat{z}+D i v(h u)=0 \text { in }[0, T] \times \mathcal{O} \\
u=0 \text { on }[0, T] \times \partial \mathcal{O} \\
u=u_{0}, \quad \hat{z}=\hat{z}_{0} \text { in }\{0\} \times \mathcal{O}
\end{array}\right.
$$

where

$$
\begin{aligned}
& f^{\prime}=f-\frac{\partial w^{0}}{\partial t}+g \nabla \int_{0}^{t} \operatorname{Div}\left(h w^{0}\right) d t-A w^{0} \\
& u_{0}(x)=w_{0}(x)-w^{0}(x, 0) \\
& \hat{z}_{0}(x)=\xi_{0}(x) .
\end{aligned}
$$

\section{Functional Setting}

We use the (vector-valued) Sobolev spaces $\mathbb{H}_{0}^{1}(\mathcal{O}):=H_{0}^{1}\left(\mathcal{O}, \mathbb{R}^{2}\right)$ and $\mathbb{L}^{2}(\mathcal{O}):=L^{2}\left(\mathcal{O}, \mathbb{R}^{2}\right)$, with: the norm on $\mathbb{H}_{0}^{1}(\mathcal{O})$ as

and the norm on $\mathbb{L}^{2}(\mathcal{O})$ as

$$
\|v\|_{\mathbb{H}_{0}^{1}}:=\left(\int_{\mathcal{O}}|\nabla v|^{2} d x\right)^{1 / 2}
$$

$$
\|v\|_{\mathbb{L}^{2}}:=\left(\int_{\mathcal{O}}|v|^{2} d x\right)^{1 / 2} .
$$

Using the Gelfand triple $\mathbb{H}_{0}^{1}(\mathcal{O}) \subset \mathbb{L}^{2}(\mathcal{O}) \subset \mathbb{H}^{-1}(\mathcal{O})$, we may consider $\triangle$ or $\nabla$ as a linear map from $\mathbb{H}_{0}^{1}(\mathcal{O})$ or $\mathbb{L}^{2}(\mathcal{O})$ into the dual of $\mathbb{H}_{0}^{1}(\mathcal{O})$ respectively. The inner product in $\mathbb{L}^{2}(\mathcal{O})$ is denoted by $(\cdot, \cdot)_{\mathbb{L}^{2}}$ and is defined by

$$
(u, v)_{\mathbb{L}^{2}}=\int_{\mathcal{O}} u(x) \cdot v(x) d x
$$

for any $u$ and $v$ in $\mathbb{L}^{2}(\mathcal{O})$. Likewise, inner product in $L^{2}(\mathcal{O})$ is denoted by $(\cdot, \cdot)_{L^{2}}$. The induced duality between the spaces $\mathbb{H}_{0}^{1}(\mathcal{O})$ and $\mathbb{H}^{-1}(\mathcal{O})$ is denoted by $\langle\cdot, \cdot\rangle$.

Lemma 3.1. For any real-valued smooth function $\varphi$ and $\psi$ with compact support in $\mathbb{R}^{2}$, the following hold:

$$
\begin{aligned}
& \|\varphi \psi\|_{L^{2}}^{2} \leq 4\left\|\varphi \partial_{1} \varphi\right\|_{L^{2}}\left\|\psi \partial_{2} \psi\right\|_{L^{2}} \\
& \|\varphi\|_{L^{4}}^{4} \leq 2\|\varphi\|_{L^{2}}^{2}\|\nabla \varphi\|_{L^{2}}^{2} .
\end{aligned}
$$

For proof see Ladyzhenskaya [25].

Notice that by means of the Gelfand triple we may consider $A$, given by (2.3), as a mapping of $\mathbb{H}_{0}^{1}(\mathcal{O})$ into its dual $\mathbb{H}^{-1}(\mathcal{O})$.

Define the non-symmetric bilinear form

$$
a(u, v):=\alpha\left[\left(\partial_{1} u_{1}, \partial_{1} v_{1}\right)_{L^{2}}+\left(\partial_{2} u_{2}, \partial_{2} v_{2}\right)_{L^{2}}\right]+\beta\left[\left(u_{1}, v_{2}\right)_{L^{2}}-\left(u_{2}, v_{1}\right)_{L^{2}}\right],
$$

on $\mathbb{H}_{0}^{1}$. Thus if $u$ has a smooth second derivative then

$$
a(u, v)=(A u, v)_{\mathbb{L}^{2}},
$$

for every $v$ in $\mathbb{H}_{0}^{1}(\mathcal{O})$. Moreover, the bilinear form $a(\cdot, \cdot)$ is continuous and coercive in $\mathbb{H}_{0}^{1}(\mathcal{O})$, i.e.,

$$
\begin{aligned}
& |a(u, v)| \leq C_{1}\|u\|_{\mathbb{H}_{0}^{1}}\|v\|_{\mathbb{H}_{0}^{1}} \quad \forall u, v \in \mathbb{H}_{0}^{1}(\mathcal{O}), \\
& (A u, u)_{\mathbb{L}^{2}}=a(u, u)=\alpha\|u\|_{\mathbb{H}_{0}^{1}}^{2}
\end{aligned}
$$

for some positive constant $C_{1}=\alpha+\beta$.

Let us denote the nonlinear operator $B(\cdot)$ by

$$
v \mapsto B(v):=\gamma\left|v+w^{0}\right|\left[v+w^{0}\right] .
$$

Then we have the following lemma: 
Lemma 3.2. Let $u$ and $v$ be in $\mathbb{L}^{4}\left(\mathcal{O}, \mathbb{R}^{2}\right)$. Then the following estimate holds:

$$
\langle B(u)-B(v), u-v\rangle \geq 0 .
$$

For proof see Lemma 3.3 in Manna, Menaldi and Sritharan 31.

The nonlinear operator $B(\cdot)$ is a continuous operator from $\mathbb{L}^{4}(\mathcal{O})$ to $\mathbb{L}^{2}(\mathcal{O})$, where

$$
\begin{aligned}
& \|B(v)\|_{\mathbb{L}^{2}} \leq C_{2}\left\|v+w^{0}\right\|_{\mathbb{L}^{4}}^{2}, \\
& \|B(u)-B(v)\|_{\mathbb{L}^{2}} \leq C_{2}\left[\left\|u+w^{0}\right\|_{\mathbb{L}^{4}}+\left\|v+w^{0}\right\|_{\mathbb{L}^{4}}\right]\|u-v\|_{\mathbb{L}^{4}},
\end{aligned}
$$

where the constant $C_{2}$ is the sup-norm of the function $\gamma$.

3.1. Preliminaries on Stochastic Processes. In this Subsection we provide definitions and some properties of Hilbert space valued Wiener processes, Lévy processes and Skorokhod spaces, most of which have been borrowed from the books by Da Prato and Zabczyk [17], Applebaum [3] and Métivier 34 .

Let $U$ and $V$ be two separable Hilbert spaces and let $\mathcal{L}(U, V)$ denote the space of all bounded linear operators from $U$ to $V$. Let $\left\{e_{j}\right\}_{j=1}^{\infty}$ be an orthonormal basis in $U$. Then we say that a positive operator $Q \in \mathcal{L}(U, U)$ (i.e., $(Q x, x)_{U} \geq 0$ for all non-zero $\left.x \in U\right)$ is trace class if $\sum_{j=1}^{\infty}\left(Q e_{j}, e_{j}\right)_{U}<\infty$. Let $Q$ be a symmetric (i.e., $\left.Q^{*}=Q\right)$, positive, trace class operator on $U$.

Then there exist a sequence of eigenvalues $\lambda_{j}$ with the corresponding sequence of eigenvectors $\left\{e_{j}\right\}$ such that $Q e_{j}=\lambda_{j} e_{j}$ for all $j=1,2, \cdots$ and

$$
\operatorname{Tr}(Q)=\sum_{j=1}^{\infty}\left(Q e_{j}, e_{j}\right)_{U}=\sum_{j=1}^{\infty}\left(\lambda_{j} e_{j}, e_{j}\right)_{U}=\sum_{j=1}^{\infty} \lambda_{j}\left\|e_{j}\right\|_{U}^{2}=\sum_{j=1}^{\infty} \lambda_{j}<\infty .
$$

Definition 3.3. Let $U$ be a Hilbert space. A stochastic process $\{W(t)\}_{0 \leq t \leq T}$ is said to be a $U$-valued $\mathcal{F}_{t}$-adapted Wiener process with covariance operator $Q$ if

(i) For each non-zero $h \in U,\left|Q^{\frac{1}{2}} h\right|^{-1}(W(t), h)_{U}$ is a standard one-dimensional Wiener process,

(ii) For any $h \in U,(W(t), h)_{U}$ is a martingale adapted to $\mathcal{F}_{t}$.

If $W$ is a $U$-valued Wiener process with covariance operator $Q$ with $\operatorname{Tr} Q<\infty$, then $W$ is a Gaussian process on $U$ and $\mathbb{E}(W(t))=0$, Cov $(W(t))=t Q, t \geq 0$. Let $U_{0}=Q^{\frac{1}{2}} U$. Then $U_{0}$ is a Hilbert space equipped with the inner product $(\cdot, \cdot)_{0}$,

$$
(u, v)_{0}=\sum_{k=1}^{\infty} \frac{1}{\lambda_{k}}\left(u, e_{k}\right)_{U}\left(v, e_{k}\right)_{U}=\left(Q^{-\frac{1}{2}} u, Q^{-\frac{1}{2}} v\right)_{U}, \forall u, v \in U_{0},
$$

where $Q^{-\frac{1}{2}}$ is the pseudo-inverse of $Q^{\frac{1}{2}}$. Since $Q$ is a trace class operator, the imbedding of $U_{0}$ in $U$ is Hilbert-Schmidt.

Let $\mathcal{L}_{Q}(U, V)$ denote the space of all Hilbert-Schmidt operator from $U_{0}$ to $V$. Let $\left\{e_{j}\right\}_{j=1}^{\infty}$, $\left\{g_{j}\right\}_{j=1}^{\infty}=\left\{\lambda_{j}^{\frac{1}{2}} e_{j}\right\}_{j=1}^{\infty}$ and $\left\{f_{j}\right\}_{j=1}^{\infty}$ be orthonormal bases for $U, U_{0}$ and $V$ respectively. Then the space $\mathcal{L}_{Q}(U, V)$ is also a separable Hilbert space, equipped with the norm

$$
\begin{aligned}
\|\Psi\|_{\mathcal{L}_{Q}(U, V)}^{2} & =\sum_{h=1}^{\infty}\left\|\Psi g_{h}\right\|_{V}^{2}=\sum_{h=1}^{\infty} \sum_{k=1}^{\infty}\left|\left(\Psi g_{h}, f_{k}\right)_{V}\right|^{2}=\sum_{h=1}^{\infty} \sum_{k=1}^{\infty}\left|\left(\Psi \lambda_{h}^{\frac{1}{2}} e_{h}, f_{k}\right)_{V}\right|^{2} \\
& =\sum_{h=1}^{\infty} \sum_{k=1}^{\infty} \lambda_{h}\left|\left(\Psi e_{h}, f_{k}\right)_{V}\right|^{2}=\sum_{h=1}^{\infty} \sum_{k=1}^{\infty}\left|\left(\Psi Q^{\frac{1}{2}} e_{h}, f_{k}\right)_{H}\right|^{2} \\
& =\sum_{h=1}^{\infty}\left\|\Psi Q^{\frac{1}{2}}\right\|_{V}^{2}=\operatorname{Tr}\left(\left(\Psi Q^{\frac{1}{2}}\right)^{*} \Psi Q^{\frac{1}{2}}\right)=\operatorname{Tr}\left(\Psi Q^{\frac{1}{2}}\left(\Psi Q^{\frac{1}{2}}\right)^{*}\right)
\end{aligned}
$$

where we used the fact that for a Hilbert-Schmidt operator $S, \operatorname{Tr}\left(S^{*} S\right)=\operatorname{Tr}\left(S S^{*}\right)$. The scalar product between two operators $\Psi, \Phi \in \mathcal{L}_{Q}(U, V)$ is defined by

$$
(\Psi, \Phi)_{\mathcal{L}_{Q}(U, V)}=\operatorname{Tr}\left(\left(\Psi Q^{\frac{1}{2}}\right)\left(\Phi Q^{\frac{1}{2}}\right)^{*}\right)
$$


Since the Hilbert spaces $U_{0}$ and $V$ are separable, the space $\mathcal{L}_{Q}(U, V)$ is also separable. For an orthonormal basis $\left\{e_{j}\right\}_{j=1}^{\infty}$ in $U$, an element $u \in U_{0}$ can be represented as

$$
u=\sum_{k=1}^{\infty}\left(u, \lambda_{k}^{\frac{1}{2}} e_{k}\right)_{0} \lambda_{k}^{\frac{1}{2}} e_{k} .
$$

Let $\Psi \in \mathcal{L}(U, V)$ be considered as an operator from $U_{0}$ to $V$, then we can write $\Psi u$ as

$$
\Psi u=\sum_{k=1}^{\infty}\left(u, \lambda_{k}^{\frac{1}{2}} e_{k}\right)_{0} \lambda_{k}^{\frac{1}{2}} \Psi e_{k} .
$$

Then $\Psi$ has a finite Hilbert-Schmidt norm, since

$$
\begin{aligned}
\|\Psi\|_{\mathcal{L}_{Q}(U, V)}^{2} & =\sum_{k=1}^{\infty}\left\|\Psi Q^{\frac{1}{2}} e_{k}\right\|_{V}^{2}=\sum_{k=1}^{\infty}\left\|\Psi \lambda_{k}^{\frac{1}{2}} e_{k}\right\|_{V}^{2}=\sum_{k=1}^{\infty} \lambda_{k}\left\|\Psi e_{k}\right\|_{V}^{2} \\
& \leq \sum_{k=1}^{\infty} \lambda_{k}\|\Psi\|_{\mathcal{L}(U, V)}^{2}\left\|e_{k}\right\|_{U}^{2}=\operatorname{Tr}(Q)\|\Psi\|_{\mathcal{L}(U, V)}^{2}
\end{aligned}
$$

and hence $\mathcal{L}(U, V) \subset \mathcal{L}_{Q}(U, V)$. Hence if $\Psi, \Phi \in \mathcal{L}(U, V)$, then from (3.13) and (3.14), we have

$$
\|\Psi\|_{\mathcal{L}_{Q}(U, V)}^{2}=\operatorname{Tr}\left(\Psi Q \Psi^{*}\right) \text { and }(\Psi, \Phi)_{\mathcal{L}_{Q}(U, V)}=\operatorname{Tr}\left(\Psi Q \Phi^{*}\right) .
$$

For more details see Da Prato and Zabczyk [17, Gawarecki and Mandrekar [20] etc.

Definition 3.4. A càdlàg adapted process (paths are right continuous with left limits), $\left(\mathbf{L}_{t}\right)_{t \geq 0}$, is called a Lévy process if it has stationary independent increments and is stochastically continuous.

Let $\left(\mathbf{L}_{t}\right)_{t \geq 0}$ be a $V$-valued Lévy process. Hence, for every $\omega \in \Omega, \mathbf{L}_{t}(\omega)$ has countable number of jumps on $[0, t]$. Note that for every $\omega \in \Omega$, the jump $\triangle \mathbf{L}_{t}(\omega)=\mathbf{L}_{t}(\omega)-\mathbf{L}_{t-}(\omega)$ is a point function in $\mathscr{B}(V \backslash\{0\})$. Let us define

$$
\begin{aligned}
N(t, Z) & =N(t, Z, \omega) \\
& :=\#\left\{s \in(0, \infty): \triangle \mathbf{L}_{s}(\omega) \in Z\right\}, t>0, Z \in \mathscr{B}(V \backslash\{0\}), \omega \in \Omega
\end{aligned}
$$

as the Poisson random measure associated with the Lévy process $\left(\mathbf{L}_{t}\right)_{t \geq 0}$.

The differential form of the measure $N(t, Z, \omega)$ is written as $N(d t, d z)(\omega)$. We call $\tilde{N}(d t, d z)=$ $N(d t, d z)-\lambda(d z) d t$ a compensated Poisson random measure (cPrm), where $\lambda(d z) d t$ is known as compensator of the Lévy process $\left(\mathbf{L}_{t}\right)_{t \geq 0}$. Here $d t$ denotes the Lebesgue measure on $\mathscr{B}\left(\mathbb{R}^{+}\right)$, and $\lambda(d z)$ is a $\sigma$-finite Lévy measure on $(Z, \mathscr{B}(Z))$.

Definition 3.5. Let $V$ and $F$ be separable Hilbert spaces. Let $F_{t}:=\mathscr{B}(V) \otimes \mathcal{F}_{t}$ be the product $\sigma$-algebra generated by the semi-ring $\mathscr{B}(V) \times \mathcal{F}_{t}$ of the product sets $Z \times F, Z \in \mathscr{B}(V), F \in \mathcal{F}_{t}$ (where $\mathcal{F}_{t}$ is the filtration of the additive process $\left(\mathbf{L}_{t}\right)_{t \geq 0}$ ). Let $T>0$, define

$$
\begin{gathered}
\mathbb{H}(Z)=\left\{g: \mathbb{R}^{+} \times Z \times \Omega \rightarrow F, \text { such that } g \text { is } F_{T} / \mathscr{B}(F)\right. \text { measurable and } \\
\left.g(t, z, \omega) \text { is } \mathcal{F}_{t} \text { - adapted } \forall z \in Z, \forall t \in(0, T]\right\} .
\end{gathered}
$$

For $p \geq 1$, let us define,

$$
\mathbb{H}_{\lambda}^{p}([0, T] \times Z ; F)=\left\{g \in \mathbb{H}(Z): \int_{0}^{T} \int_{Z} \mathbb{E}\left[\|g(t, z, \omega)\|_{F}^{p}\right] \lambda(d z) d t<\infty\right\} .
$$

For more details see Mandrekar and Rüdiger 27.

Let $S$ be a complete separable metric space with a metric $d$. Let us denote by $D([0, T] ; S)$, the set of all $S$-valued functions defined on $[0, T]$, which are right continuous and have left limits (càdlàg functions) for every $t \in[0, T]$. The space $D([0, T] ; S)$ is endowed with the Skorokhod $J$-topology. 
Definition 3.6. (The $J$-topology on $D([0, T] ; S)$ ) This topology can be defined by the following metric $\delta_{T}$.

$$
\begin{aligned}
& \delta_{T}(x, y) \\
& :=\inf _{\lambda \in \Lambda_{T}}\left[\sup _{t \in[0, T]} d(x(t), x \circ \lambda(t))+\sup _{t \in[0, T]}|t-\lambda(t)|+\sup _{s \neq t}\left|\log \left(\frac{\lambda(t)-\lambda(s)}{t-s}\right)\right|\right],
\end{aligned}
$$

where $\Lambda_{T}$ is the set of increasing homeomorphisms of $[0, T]$.

A sequence $\left(x_{n}\right)_{n \in \mathbb{N}}$ in $D([0, T] ; S)$ converges to $x$ in $D([0, T] ; S)$ if and only if there exists a sequence $\left(\lambda_{n}\right)$ of homeomorphisms of $[0, T]$ such that $\lim \lambda_{n}$ is equal to identity mapping and $x=\lim _{n} x_{n} \circ \lambda_{n}$, both convergence being uniform on $[0, T]$.

The Skorokhod topology relativized to $C([0, T] ; S)$ coincides with the uniform topology there. It should be remarked that, if a sequence $\left(x_{n}\right)$ in $D([0, T] ; S)$ converges to $x$ for the metric $\delta_{T}$, then $\lim x_{n}(T)=x(T)$. The space $D([0, T] ; S)$ is separable and complete. For more details see Chapter 2, Métivier [34] and Chapter 3, Billingsley [7].

Remark 3.7. Let us define $D([0, T] ; V)$ as the space of all càdlàg paths from $[0, T]$ into $V$, where $V$ is a Hilbert space.

Definition 3.8. Let $M$ be a V-valued square integrable martingale on $\left(\Omega, \mathcal{F},\left(\mathcal{F}_{t}\right)_{t \geq 0}, \mathbb{P}\right)$, with right continuous paths. Then there exists two real right continuous increasing processes $[M]$ and $\triangleleft M \triangleright$ with $0=[M]_{0}=\triangleleft M \triangleright_{0}$ such that

$$
\left\|M_{t}\right\|_{V}^{2}=\left\|M_{0}\right\|_{V}^{2}+2 \int_{0}^{t}\left(M_{s-}, d M_{s}\right)_{V}+[M]_{t} .
$$

$\triangleleft M \triangleright$ is the unique real right continuous increasing predictable process such that

$$
\left\|M_{t}\right\|_{V}^{2}-\left\|M_{0}\right\|_{V}^{2}-\triangleleft M \triangleright_{t} \text { is a martingale. }
$$

Here $[M]$ is called the quadratic variation of $M$ and $\triangleleft M \triangleright$ the Meyer process of $M$.

Remark 3.9. If $M$ is continuous, then we have $\triangleleft M \triangleright=[M]$.

Example 3.10. Let $V$ be Hilbert spaces and let $Q: V \rightarrow V$ be a trace class operator. Let

$$
d u(t)=\sigma(t, u) d W(t)+\int_{Z} g(u(t-), z) \tilde{N}(d t, d z),
$$

where $W(\cdot)$ is a $V$-valued Wiener process, $\sigma(\cdot, \cdot):[0, t) \times V \rightarrow \mathcal{L}_{Q}(V, V), Z \in \mathscr{B}(V \backslash\{0\}), g(\cdot, \cdot)$ : $V \times Z \rightarrow V$ and $\tilde{N}(\cdot, \cdot)$ is the compensated Poisson random measure. From Métivier [34, Sakthivel and Sritharan [43] and Manna, Manil and Sritharan [30], we observe that the quadratic variation process of $u$ is

$$
[u]_{t}=\int_{0}^{t}\|\sigma(s, u)\|_{\mathcal{L}_{Q}(V, V)}^{2} d s+\int_{0}^{t} \int_{Z}\|g(u, z)\|_{V}^{2} N(d s, d z)
$$

and the Meyer process of $u$ is

$$
\triangleleft u \triangleright_{t}=\int_{0}^{t}\|\sigma(s, u)\|_{\mathcal{L}_{Q}(V, V)}^{2} d s+\int_{0}^{t} \int_{Z}\|g(u, z)\|_{V}^{2} \lambda(d z) d s,
$$

and finally by the martingale property one obtains that the expectation of the quadratic variation process and that of Meyer process are same. This yields

$$
\mathbb{E}\left[\int_{0}^{t} \int_{Z}\|g(u, z)\|_{V}^{2} N(d s, d z)\right]=\mathbb{E}\left[\int_{0}^{t} \int_{Z}\|g(u, z)\|_{V}^{2} \lambda(d z) d s\right] .
$$

Lemma 3.11. (Burkholder-Davis-Gundy Inequality) Let $M$ be a $V$-valued càdlàg martingale with $M_{0}=0$ and let $p \geq 1$ be fixed. Then for any $\mathcal{F}$-stopping time $\tau$, there exists constants $c_{p}$ and $C_{p}$ such that

$$
\mathbb{E}\left\{[M]_{\tau}^{p / 2}\right\} \leq c_{p} \mathbb{E}\left\{\sup _{0 \leq t \leq \tau}\left\|M_{t}\right\|_{V}^{p}\right\} \leq C_{p} \mathbb{E}\left\{[M]_{\tau}^{p / 2}\right\}
$$


for all $\tau, 0 \leq \tau \leq \infty$, where $[M]$ is the quadratic variation of process $M$. The constants are universal, they do not depend on the choice of $M$.

For proof see Theorem 1.1 of Marinelli and Röckner [33. For real-valued càdlàg martingales see Theorem 3.50 of Peszat and Zabczyk [40].

3.2. Stochastic Tide Model. Let us consider $U=V=\mathbb{L}^{2}$. Then with the above functional setting, we recall the stochastic tidal dynamics equation (1.1)-(1.3) with the Lévy forcing where $u_{0} \in L^{2}\left(\Omega ; \mathbb{L}^{2}(\mathcal{O})\right)$, and $\hat{z}_{0} \in L^{2}\left(\Omega ; L^{2}(\mathcal{O})\right)$. The operators $A$ and $B$ are defined through (2.3) and (3.9) respectively. $(W(t))_{t \geq 0}$ is a $\mathbb{L}^{2}(\mathcal{O})$-valued Wiener process with trace class covariance. $H(\cdot, \cdot)$ is a measurable mapping from $\mathbb{H}_{0}^{1} \times Z$ into $\mathbb{L}^{2}$.

Assumption 3.12. We assume that $\sigma$ and $H$ satisfy the following hypotheses:

H.1 $\sigma \in C\left([0, T] \times \mathbb{H}_{0}^{1}(\mathcal{O}) ; L_{Q}\left(\mathbb{L}^{2}, \mathbb{L}^{2}\right)\right), H \in \mathbb{H}_{\lambda}^{2}\left([0, T] \times Z ; \mathbb{L}^{2}(\mathcal{O})\right)$.

H.2 For all $t \in(0, T)$, there exists a positive constant $K$ such that for all $u \in \mathbb{L}^{2}(\mathcal{O})$

$$
\|\sigma(t, u)\|_{L_{Q}\left(\mathbb{L}^{2}, \mathbb{L}^{2}\right)}^{2}+\int_{Z}\|H(u, z)\|_{\mathbb{L}^{2}}^{2} \lambda(d z) \leq K\left(1+\|u\|_{\mathbb{L}^{2}}^{2}\right) .
$$

H.3 For all $t \in(0, T)$, there exists a positive constant $L$ such that for all $u, v \in \mathbb{L}^{2}(\mathcal{O})$

$$
\|\sigma(t, u)-\sigma(t, v)\|_{L_{Q}\left(\mathbb{L}^{2}, \mathbb{L}^{2}\right)}^{2}+\int_{Z}\|H(u, z)-H(v, z)\|_{\mathbb{L}^{2}}^{2} \lambda(d z) \leq L\|u-v\|_{\mathbb{L}^{2}}^{2} .
$$

Lemma 3.13. Let $F(\cdot): \mathbb{H}_{0}^{1} \rightarrow \mathbb{H}^{-1}$ be a nonlinear operator defined by $F(u):=A u+B(u)-f$. Then for $u, v \in \mathbb{H}_{0}^{1}(\mathcal{O})$,

$$
\langle F(u)-F(v), h u-h v\rangle+\Lambda\|u-v\|_{\mathbb{H}_{0}^{1}}^{2} \geq 0,
$$

where $\Lambda:=\Lambda(\alpha, \epsilon, M)$ is a real constant and $\epsilon$ and $M$ are defined in (2.5) and $\alpha$ is defined via the definition of the operator $A$.

Proof. From the definition of the operator A, assumptions (2.5) on $h(x)$, applying Young's and Poincaré inequalities, we obtain

$$
\begin{aligned}
\langle A u, h u\rangle & =\alpha(\nabla u, h \nabla u)_{\mathbb{L}^{2}}+\alpha(\nabla u, u \nabla h)_{\mathbb{L}^{2}} \geq \alpha \epsilon\|\nabla u\|_{\mathbb{L}^{2}}^{2}-\alpha M\|\nabla u\|_{\mathbb{L}^{2}}\|u\|_{\mathbb{L}^{2}} \\
& \geq \frac{\alpha \epsilon}{2}\|\nabla u\|_{\mathbb{L}^{2}}^{2}-\frac{\alpha M^{2}}{2 \epsilon}\|u\|_{\mathbb{L}^{2}}^{2} \geq\left(\frac{\alpha \epsilon}{2}-\frac{\alpha M^{2}}{2 \epsilon \lambda_{1}}\right)\|u\|_{\mathbb{H}_{0}^{1}}^{2},
\end{aligned}
$$

where $\lambda_{1}$ is the first eigenvalue of $-\triangle$. Hence for any $u, v \in \mathbb{H}_{0}^{1}(\mathcal{O})$,

$$
\langle A u-A v, h u-h v\rangle \geq\left(\frac{\alpha \epsilon}{2}-\frac{\alpha M^{2}}{2 \epsilon \lambda_{1}}\right)\|u-v\|_{\mathbb{H}_{0}^{1}}^{2} .
$$

From Lemma 3.2. we can easily show that for $u, v \in \mathbb{H}_{0}^{1}(\mathcal{O}) \subset \mathbb{L}^{4}(\mathcal{O})$

$$
\langle B(u)-B(v), h u-h v\rangle \geq 0 .
$$

Hence (3.19) is obvious with $\Lambda:=\frac{\alpha}{2 \epsilon}\left(\frac{M^{2}}{\lambda_{1}}-\epsilon^{2}\right)$.

Remark 3.14. (monotonicity in $\mathbb{H}_{0}^{1}$ ): If we assume $\Lambda<0$, then the operator $F(\cdot)$ is monotone on $\mathbb{H}_{0}^{1}(\mathcal{O})$. Moreover, since $F(\cdot)$ is hemicontinuous operator, it is maximal monotone from $\mathbb{H}_{0}^{1}(\mathcal{O})$ to $\mathbb{H}^{-1}(\mathcal{O})$ (see Theorem 1.3 of Barbu [6]).

However, in general, if we do not assume negativity of the constant $\Lambda$, then the operator $F(\cdot)$ is $\Lambda$ - monotone on $\mathbb{H}_{0}^{1}(\mathcal{O})$ i.e. $F+\Lambda I$ is monotone on $\mathbb{H}_{0}^{1}(\mathcal{O})$.

Remark 3.15. (monotonicity in $\mathbb{L}^{2}$ ): Use of Young's inequality with different parameters can lead to

$$
\begin{aligned}
\langle A u, h u\rangle & =\alpha(\nabla u, h \nabla u)_{\mathbb{L}^{2}}+\alpha(\nabla u, u \nabla h)_{\mathbb{L}^{2}} \geq \alpha \epsilon\|\nabla u\|_{\mathbb{L}^{2}}^{2}-\alpha M\|\nabla u\|_{\mathbb{L}^{2}}\|u\|_{\mathbb{L}^{2}} \\
& \geq \frac{\alpha \epsilon}{2}\|\nabla u\|_{\mathbb{L}^{2}}^{2}-\frac{\alpha \epsilon}{2}\|\nabla u\|_{\mathbb{L}^{2}}^{2}-\frac{\alpha M^{2}}{2 \epsilon}\|u\|_{\mathbb{L}^{2}}^{2},
\end{aligned}
$$


which in turn yields

$$
\langle F(u)-F(v), h u-h v\rangle+\frac{\alpha M^{2}}{2 \epsilon}\|u-v\|_{\mathbb{L}^{2}}^{2} \geq 0 .
$$

Hence $F(\cdot)$ is $\Lambda_{1}$ - monotone on $\mathbb{L}^{2}(\mathcal{O})$ (with $\Lambda_{1}:=\frac{\alpha M^{2}}{2 \epsilon}>0$ ), and this interesting property will be exploited in the proof of existence theorem in the next Section.

\section{Energy Estimates and Existence Result}

Let $\left\{e_{j}: j \in \mathbb{N}\right\}$ be an orthonormal basis in $\mathbb{L}^{2}(\mathcal{O})$ such that $\operatorname{span}\left\{e_{j}: j \in \mathbb{N}\right\}$ is dense in $\mathbb{H}_{0}^{1}(\mathcal{O})$. Let us fix $n \in \mathbb{N}$. Let $P_{n}$ denote the projection of $\mathbb{H}^{-1}(\mathcal{O})$ onto $\mathbb{L}_{n}^{2}(\mathcal{O}):=\operatorname{span}\left\{e_{1}, e_{2}, \cdots, e_{n}\right\}$ defined by

$$
P_{n} u:=\sum_{i=1}^{n}\left\langle u, e_{i}\right\rangle e_{i}, \quad u \in \mathbb{H}^{-1}(\mathcal{O})
$$

Note that the restriction of $P_{n}$ to $\mathbb{L}^{2}(\mathcal{O})$, still denoted by $P_{n}$, is given by

$$
P_{n} u:=\sum_{i=1}^{n}\left(u, e_{i}\right)_{\mathbb{L}^{2}} e_{i}, \quad u \in \mathbb{L}^{2}(\mathcal{O})
$$

and thus it is the $(\cdot, \cdot)_{\mathbb{L}^{2}}$-orthogonal projection onto $\mathbb{L}_{n}^{2}(\mathcal{O})$.

In similar manner we can denote the orthogonal projection of $L^{2}(\mathcal{O})$ to $L_{n}^{2}(\mathcal{O})$ by the same notation $P_{n}$.

Lemma 4.1. For every $u \in \mathbb{L}^{2}(\mathcal{O}), v \in L^{2}(\mathcal{O}), w \in \mathbb{H}_{0}^{1}(\mathcal{O})$,

$$
\lim _{n \rightarrow \infty}\left\|P_{n} w-w\right\|_{\mathbb{H}_{0}^{1}}=0, \lim _{n \rightarrow \infty}\left\|P_{n} u-u\right\|_{\mathbb{L}^{2}}=0, \lim _{n \rightarrow \infty}\left\|P_{n} v-v\right\|_{L^{2}}=0
$$

Proof. See Lemma 2.4 of [1].

For every $n \in \mathbb{N}$, we consider the finite dimensional system of SDEs in variational form on $\mathbb{L}_{n}^{2}(\mathcal{O})$ and $L_{n}^{2}(\mathcal{O})$ respectively given by

$$
\begin{aligned}
& d u^{n}(t)+\left(A u^{n}(t)+B\left(u^{n}(t)\right)+g \nabla \hat{z}^{n}(t)\right) d t=f(t) d t \\
& \quad+\sigma^{n}\left(t, u^{n}(t)\right) d W^{n}(t)+\int_{Z} H^{n}\left(u^{n}(t-), z\right) \tilde{N}(d t, d z) \\
& d \hat{z}^{n}(t)+D i v\left(h u^{n}(t)\right) d t=0, \\
& u^{n}(0)=P_{n} u_{0}=u_{0}^{n}, \quad \hat{z}^{n}(0)=P_{n} \hat{z}_{0}=\hat{z}_{0}^{n},
\end{aligned}
$$

where we have denoted $W^{n}=P_{n} W, \sigma^{n}=P_{n} \sigma$, and $\int_{Z} H^{n}(\cdot, z) \tilde{N}(d t, d z)=P_{n} \int_{Z} H(\cdot, z) \tilde{N}(d t, d z)$ with $H^{n}=P_{n} H$. Due to "weak" nonlinearity of $B$, it is straightforward to show that the range of $B\left(u^{n}\right)$ and that of $P_{n} B\left(u^{n}\right)$ are $\mathbb{L}_{n}^{2}(\mathcal{O})$, and hence we write $P_{n} B\left(u^{n}\right)$ as $B\left(u^{n}\right)$. For simplicity, we write $P_{n} f$ as $f$ in equation (4.1), since due to Lemma $4.1 P_{n} f \rightarrow f$ in $L^{2}\left(0, T ; \mathbb{L}^{2}(\mathcal{O})\right)$.

Remark 4.2. We note that (3.12) ensures that $B$ is locally Lipschitz and Assumption 3.12 guarantees that the noise coefficients $\sigma^{n}$ and $H^{n}$ are globally Lipschitz. Hence, we infer that for all $n \geq 1$, there exist adapted processes $u^{n} \in D\left([0, T] ; \mathbb{L}_{n}^{2}(\mathcal{O})\right)$ a.s. and $\hat{z}^{n} \in C\left([0, T] ; L_{n}^{2}(\mathcal{O})\right)$ a.s. such that (4.1)-(4.3) are satisfied. For proof see Albeverio et. al [4].

Proposition 4.3. Under the above mathematical setting, let

$$
\left\{\begin{array}{l}
w^{0} \in L^{4}\left(\Omega ; L^{4}\left(0, T ; \mathbb{L}^{4}(\mathcal{O})\right)\right), f \in L^{2}\left(\Omega ; L^{2}\left(0, T ; \mathbb{L}^{2}(\mathcal{O})\right)\right), \\
\sigma \in C\left([0, T] \times \mathbb{H}_{0}^{1}(\mathcal{O}) ; L_{Q}\left(\mathbb{L}^{2}, \mathbb{L}^{2}\right)\right), H \in \mathbb{H}_{\lambda}^{2}\left([0, T] \times Z ; \mathbb{L}^{2}(\mathcal{O})\right), \\
u_{0} \in L^{2}\left(\Omega ; \mathbb{L}^{2}(\mathcal{O})\right), \hat{z}_{0} \in L^{2}\left(\Omega ; L^{2}(\mathcal{O})\right) .
\end{array}\right.
$$

If $\left(u^{n}(t), \hat{z}^{n}(t)\right)$ denotes the unique strong solution of the system (4.1)-(4.3), then we have the following a-priori estimates:

$$
\mathbb{E}\left[\left\|u^{n}(t)\right\|_{\mathbb{L}^{2}}^{2}+\left\|\hat{z}^{n}(t)\right\|_{L^{2}}^{2}\right]+2 \alpha \mathbb{E}\left[\int_{0}^{t}\left\|u^{n}(s)\right\|_{\mathbb{H}_{0}^{1}}^{2} d s\right] \leq C_{1(2)} \quad \forall t \in[0, T],
$$




$$
\mathbb{E}\left[\sup _{0 \leq t \leq T}\left(\left\|u^{n}(t)\right\|_{\mathbb{L}^{2}}^{2}+\left\|\hat{z}^{n}(t)\right\|_{L^{2}}^{2}\right)\right]+2 \alpha \mathbb{E}\left[\int_{0}^{T}\left\|u^{n}(t)\right\|_{\mathbb{H}_{0}^{1}}^{2} d t\right] \leq C_{2(2)},
$$

where the constants $C_{1(2)}$ and $C_{2(2)}$ depend on the coefficients $\alpha, g, M, \mu$ and the norms $\|f\|_{L^{2}\left(\Omega ; L^{2}\left(0, T ; \mathbb{L}^{2}\right)\right)}$, $\left\|w^{0}\right\|_{L^{4}\left(\Omega ; L^{4}\left(0, T ; \mathbb{L}^{4}\right)\right)},\left\|u_{0}\right\|_{L^{2}\left(\Omega ; \mathbb{L}^{2}\right)},\left\|\hat{z}_{0}\right\|_{L^{2}\left(\Omega ; L^{2}\right)}$ and $T$.

Proof. Define the stopping time as:

$$
\tau_{N}=\inf \left\{t \geq 0:\left\|u^{n}(t)\right\|_{\mathbb{L}^{2}}^{2}+\left\|\hat{z}^{n}(t)\right\|_{L^{2}}^{2}+\int_{0}^{t}\left\|u^{n}(s)\right\|_{\mathbb{H}_{0}^{1}}^{2} d s>N\right\} .
$$

Applying Itô's lemma (for reference, see Theorem 3.7.2 of Mandrekar and Rüdiger [28, Theorem 4.4 of Rüdiger and Ziglio [42], Section 2.3, Métivier [34]) to the process $\left\|u^{n}(t)\right\|_{\mathbb{L}^{2}}^{2}$

$$
\begin{aligned}
& \left\|u^{n}\left(t \wedge \tau_{N}\right)\right\|_{\mathbb{L}^{2}}^{2}+2 \alpha \int_{0}^{t \wedge \tau_{N}}\left\|u^{n}(s)\right\|_{\mathbb{H}_{0}^{1}}^{2} d s+2 \int_{0}^{t \wedge \tau_{N}}\left(B\left(u^{n}(s)\right), u^{n}(s)\right)_{\mathbb{L}^{2}} d s \\
& \quad+2 \int_{0}^{t \wedge \tau_{N}}\left(g \nabla \hat{z}^{n}(s), u^{n}(s)\right)_{\mathbb{L}^{2}} d s=\left\|u_{0}^{n}\right\|_{\mathbb{L}^{2}}^{2}+2 \int_{0}^{t \wedge \tau_{N}}\left(f(s), u^{n}(s)\right)_{\mathbb{L}^{2}} d s \\
& \quad+2 \int_{0}^{t \wedge \tau_{N}}\left(\sigma^{n}\left(s, u^{n}(s)\right) d W^{n}(s), u^{n}(s)\right)_{\mathbb{L}^{2}}+\int_{0}^{t \wedge \tau_{N}}\left\|\sigma^{n}\left(s, u^{n}(s)\right)\right\|_{L_{Q}\left(\mathbb{L}^{2} ; \mathbb{L}^{2}\right)}^{2} d s \\
& \quad+\int_{0}^{t \wedge \tau_{N}} \int_{Z}\left\|H^{n}\left(u^{n}(s-), z\right)\right\|_{\mathbb{L}^{2}}^{2} N(d s, d z) \\
& \quad+2 \int_{0}^{t \wedge \tau_{N}} \int_{Z}\left(H^{n}\left(u^{n}(s-), z\right), u^{n}(s-)\right)_{\mathbb{L}^{2}} \tilde{N}(d s, d z) .
\end{aligned}
$$

Using the definition of the operator $B(\cdot)$ and Lemma 3.2

$$
\begin{aligned}
\left(B\left(u^{n}(t)\right), u^{n}(t)\right)_{\mathbb{L}^{2}} & \geq-\int_{\mathcal{O}} \gamma(x)\left|w^{0}(t)\right| w^{0}(t) u^{n}(t) d x \\
& \geq-\frac{r}{\epsilon}\left\|w^{0}(t)\right\|_{\mathbb{L}^{4}}^{2}\left\|u^{n}(t)\right\|_{\mathbb{L}^{2}} \\
& \geq-\frac{r}{2 \epsilon}\left[\left\|w^{0}(t)\right\|_{\mathbb{L}^{4}}^{4}+\left\|u^{n}(t)\right\|_{\mathbb{L}^{2}}^{2}\right] .
\end{aligned}
$$

Using the Divergence Theorem and the inequality $2 a b \leq \delta a^{2}+\frac{1}{\delta} b^{2}$ we obtain,

$$
\begin{aligned}
& \left|g\left(\nabla \hat{z}^{n}(t), u^{n}(t)\right)_{\mathbb{L}^{2}}\right|=\left|-g\left(\hat{z}^{n}(t), \operatorname{Div}\left(u^{n}(t)\right)\right)_{L^{2}}\right| \\
& \leq \frac{g}{2}\left[\frac{2 g}{\alpha}\left\|\hat{z}^{n}(t)\right\|_{L^{2}}^{2}+\frac{\alpha}{2 g}\left\|\operatorname{Div}\left(u^{n}(t)\right)\right\|_{L^{2}}^{2}\right] \\
& \leq \frac{g}{2}\left[\frac{2 g}{\alpha}\left\|\hat{z}^{n}(t)\right\|_{L^{2}}^{2}+\frac{\alpha}{2 g}\left\|u^{n}(t)\right\|_{\mathbb{H}_{0}^{1}}^{2}\right] .
\end{aligned}
$$

Using Cauchy-Schwarz inequality

$$
\left|\left(f(t), u^{n}(t)\right)_{\mathbb{L}^{2}}\right| \leq \frac{1}{2}\left[\|f(t)\|_{\mathbb{L}^{2}}^{2}+\left\|u^{n}(t)\right\|_{\mathbb{L}^{2}}^{2}\right] .
$$

Hence the energy equality (4.8) yields

$$
\begin{aligned}
& \left\|u^{n}\left(t \wedge \tau_{N}\right)\right\|_{\mathbb{L}^{2}}^{2}+2 \alpha \int_{0}^{t \wedge \tau_{N}}\left\|u^{n}(s)\right\|_{\mathbb{H}_{0}^{1}}^{2} d s \\
& \leq \int_{0}^{t \wedge \tau_{N}}\|f(s)\|_{\mathbb{L}^{2}}^{2} d s+\int_{0}^{t \wedge \tau_{N}}\left\|u^{n}(s)\right\|_{\mathbb{L}^{2}}^{2} d s+\frac{2 g^{2}}{\alpha} \int_{0}^{t \wedge \tau_{N}}\left\|\hat{z}^{n}(s)\right\|_{L^{2}}^{2} d s \\
& \quad+\frac{r}{\epsilon} \int_{0}^{t \wedge \tau_{N}}\left[\left\|w^{0}(s)\right\|_{\mathbb{L}^{4}}^{4}+\left\|u^{n}(s)\right\|_{\mathbb{L}^{2}}^{2}\right] d s+\frac{\alpha}{2} \int_{0}^{t \wedge \tau_{N}}\left\|u^{n}(s)\right\|_{\mathbb{H}_{0}^{1}}^{2} d s \\
& \quad+2 \int_{0}^{t \wedge \tau_{N}}\left(\sigma^{n}\left(s, u^{n}(s)\right) d W^{n}(s), u^{n}(s)\right)_{\mathbb{L}^{2}}+\int_{0}^{t \wedge \tau_{N}}\left\|\sigma^{n}\left(s, u^{n}(s)\right)\right\|_{L_{Q}\left(\mathbb{L}^{2} ; \mathbb{L}^{2}\right)}^{2} d s \\
& \quad+\int_{0}^{t \wedge \tau_{N}} \int_{Z}\left\|H^{n}\left(u^{n}(s-), z\right)\right\|_{\mathbb{L}^{2}}^{2} N(d s, d z)+\left\|u_{0}^{n}\right\|_{\mathbb{L}^{2}}^{2}
\end{aligned}
$$




$$
+2 \int_{0}^{t \wedge \tau_{N}} \int_{Z}\left(H^{n}\left(u^{n}(s-), z\right), u^{n}(s-)\right)_{\mathbb{L}^{2}} \tilde{N}(d s, d z) .
$$

Using equation (4.2)

$$
\frac{1}{2} \frac{d}{d t}\left\|\hat{z}^{n}(t)\right\|_{L^{2}}^{2}=-\left(\operatorname{Div}\left(h u^{n}(t)\right), \hat{z}^{n}(t)\right)_{L^{2}} .
$$

Now by using Hölder's inequality, assumptions on $h$ in (2.5) and Young's inequality we get

$$
\begin{aligned}
& \left|\left(\operatorname{Div}\left(h u^{n}(t)\right), \hat{z}^{n}(t)\right)_{L^{2}}\right|=\left|\left(h \operatorname{Div}\left(u^{n}(t)\right), \hat{z}^{n}(t)\right)_{L^{2}}+\left(u^{n}(t) \cdot \nabla h, \hat{z}^{n}(t)\right)_{L^{2}}\right| \\
& \leq\left|\left(h \operatorname{Div}\left(u^{n}(t)\right), \hat{z}^{n}(t)\right)_{L^{2}}\right|+\left|\left(u^{n}(t) \cdot \nabla h, \hat{z}^{n}(t)\right)_{L^{2}}\right| \\
& \leq\|h\|_{L^{\infty}}\left\|\operatorname{Div}\left(u^{n}(t)\right)\right\|_{L^{2}}\left\|\hat{z}^{n}(t)\right\|_{L^{2}}+\left\|u^{n}(t)\right\|_{\mathbb{L}^{2}}\|\nabla h\|_{\mathbb{L}^{\infty}}\left\|\hat{z}^{n}(t)\right\|_{L^{2}} \\
& \leq \mu\left\|u^{n}(t)\right\|_{\mathbb{H}_{0}^{1}}\left\|\hat{z}^{n}(t)\right\|_{L^{2}}+M\left\|u^{n}(t)\right\|_{\mathbb{L}^{2}}\left\|\hat{z}^{n}(t)\right\|_{L^{2}} \\
& \leq \frac{\mu}{2}\left(\frac{\alpha}{2 \mu}\left\|u^{n}(t)\right\|_{\mathbb{H}_{0}^{1}}^{2}+\frac{2 \mu}{\alpha}\left\|\hat{z}^{n}(t)\right\|_{L^{2}}^{2}\right)+\frac{M}{2}\left[\left\|u^{n}(t)\right\|_{\mathbb{L}^{2}}^{2}+\left\|\hat{z}^{n}(t)\right\|_{L^{2}}^{2}\right] .
\end{aligned}
$$

Thus integrating (4.13) in $\left[0, t \wedge \tau_{N}\right]$ and using (4.14) we get

$$
\begin{aligned}
\left\|\hat{z}^{n}\left(t \wedge \tau_{N}\right)\right\|_{L^{2}}^{2} \leq & \left\|\hat{z}^{n}(0)\right\|_{L^{2}}^{2}+M \int_{0}^{t \wedge \tau_{N}}\left\|u^{n}(s)\right\|_{\mathbb{L}^{2}}^{2} d s \\
& +\left(\frac{2 \mu^{2}}{\alpha}+M\right) \int_{0}^{t \wedge \tau_{N}}\left\|\hat{z}^{n}(s)\right\|_{L^{2}}^{2} d s+\frac{\alpha}{2} \int_{0}^{t \wedge \tau_{N}}\left\|u^{n}(s)\right\|_{\mathbb{H}_{0}^{1}}^{2} d s .
\end{aligned}
$$

Adding equations (4.12) and (4.15)

$$
\begin{aligned}
& \left\|u^{n}\left(t \wedge \tau_{N}\right)\right\|_{\mathbb{L}^{2}}^{2}+\left\|\hat{z}^{n}\left(t \wedge \tau_{N}\right)\right\|_{L^{2}}^{2}+\alpha \int_{0}^{t \wedge \tau_{N}}\left\|u^{n}(s)\right\|_{\mathbb{H}_{0}^{1}}^{2} d s \\
& \leq\left(1+M+\frac{r}{\epsilon}\right) \int_{0}^{t \wedge \tau_{N}}\left\|u^{n}(s)\right\|_{\mathbb{L}^{2}}^{2} d s+\left(\frac{2 g^{2}}{\alpha}+\frac{2 \mu^{2}}{\alpha}+M\right) \int_{0}^{t \wedge \tau_{N}}\left\|\hat{z}^{n}(s)\right\|_{L^{2}}^{2} d s \\
& \quad+\frac{r}{\epsilon} \int_{0}^{t \wedge \tau_{N}}\left\|w^{0}(s)\right\|_{\mathbb{L}^{4}}^{4} d s+\int_{0}^{t \wedge \tau_{N}}\|f(s)\|_{\mathbb{L}^{2}}^{2} d s+\int_{0}^{t \wedge \tau_{N}}\left\|\sigma^{n}\left(s, u^{n}(s)\right)\right\|_{L_{Q}\left(\mathbb{L}^{2} ; \mathbb{L}^{2}\right)}^{2} d s \\
& \quad+2 \int_{0}^{t \wedge \tau_{N}}\left(\sigma^{n}\left(s, u^{n}(s)\right) d W^{n}(s), u^{n}(s)\right)_{\mathbb{L}^{2}}+\left\|u_{0}^{n}\right\|_{\mathbb{L}^{2}}^{2}+\left\|\hat{z}_{0}^{n}\right\|_{L^{2}}^{2} \\
& \quad+\int_{0}^{t \wedge \tau_{N}} \int_{Z}\left\|H^{n}\left(u^{n}(s-), z\right)\right\|_{\mathbb{L}^{2}}^{2} N(d s, d z) \\
& \quad+\int_{0}^{t \wedge \tau_{N}} 2 \int_{Z}\left(H^{n}\left(u^{n}(s-), z\right), u^{n}(s-)\right)_{\mathbb{L}^{2}} \tilde{N}(d s, d z) .
\end{aligned}
$$

Let $C=\max \left\{1+M+\frac{r}{\epsilon}, \frac{2 g^{2}}{\alpha}+\frac{2 \mu^{2}}{\alpha}+M\right\}$. We note that the expectation of the quadratic variation process and that of Meyer process are same, i.e.

$$
\begin{aligned}
& \mathbb{E}\left[\int_{0}^{t} \int_{Z}\left\|H^{n}\left(u^{n}(s-), z\right)\right\|_{\mathbb{L}^{2}}^{2} N(d s, d z)\right] \\
& =\mathbb{E}\left[\int_{0}^{t} \int_{Z}\left\|H^{n}\left(u^{n}(s), z\right)\right\|_{\mathbb{L}^{2}}^{2} \lambda(d z) d s\right] .
\end{aligned}
$$

and the stochastic integrals

$$
\begin{aligned}
& \int_{0}^{t \wedge \tau_{N}}\left(\sigma^{n}\left(s, u^{n}(s)\right) d W^{n}(s), u^{n}(s)\right)_{\mathbb{L}^{2}}, \\
& \int_{0}^{t \wedge \tau_{N}} \int_{Z}\left(H^{n}\left(u^{n}(s-), z\right), u^{n}(s-)\right)_{\mathbb{L}^{2}} \tilde{N}(d s, d z)
\end{aligned}
$$

are local martingales with zero averages. Furthermore, we observe that

$$
\left\|\sigma^{n}\left(s, u^{n}(s)\right)\right\|_{L_{Q}\left(\mathbb{L}^{2} ; \mathbb{L}^{2}\right)} \leq c\left\|P_{n}\right\|_{\mathcal{L}\left(\mathbb{L}^{2}, \mathbb{L}^{2}\right)}\left\|\sigma\left(s, u^{n}(s)\right)\right\|_{L_{Q}\left(\mathbb{L}^{2} ; \mathbb{L}^{2}\right)} \leq c\left\|\sigma\left(s, u^{n}(s)\right)\right\|_{L_{Q}\left(\mathbb{L}^{2} ; \mathbb{L}^{2}\right)},
$$


and similarly

$$
\left\|H^{n}\left(u^{n}(s), z\right)\right\|_{\mathbb{L}^{2}} \leq c\left\|P_{n}\right\|_{\mathcal{L}\left(\mathbb{L}^{2}, \mathbb{L}^{2}\right)}\left\|H\left(u^{n}(s), z\right)\right\|_{\mathbb{L}^{2}} \leq c\left\|H\left(u^{n}(s), z\right)\right\|_{\mathbb{L}^{2}} .
$$

Hence after taking expectation of equation (4.16), using (4.18), (4.19) and Assumption 3.12 we obtain the following

$$
\begin{aligned}
\mathbb{E} & {\left[\left\|u^{n}\left(t \wedge \tau_{N}\right)\right\|_{\mathbb{L}^{2}}^{2}+\left\|\hat{z}^{n}\left(t \wedge \tau_{N}\right)\right\|_{L^{2}}^{2}\right]+\alpha \mathbb{E}\left[\int_{0}^{t \wedge \tau_{N}}\left\|u^{n}(s)\right\|_{\mathbb{H}_{0}^{1}}^{2} d s\right] } \\
\leq & C \mathbb{E}\left[\int_{0}^{t \wedge \tau_{N}}\left(\left\|u^{n}(s)\right\|_{\mathbb{L}^{2}}^{2}+\left\|\hat{z}^{n}(s)\right\|_{L^{2}}^{2}+\left\|w^{0}(s)\right\|_{\mathbb{L}^{4}}^{4}\right) d s\right] \\
& +\mathbb{E}\left[\int_{0}^{t \wedge \tau_{N}}\|f(s)\|_{\mathbb{L}^{2}}^{2} d s\right]+\mathbb{E}\left[\int_{0}^{t \wedge \tau_{N}}\left\|\sigma^{n}\left(s, u^{n}(s)\right)\right\|_{L_{Q}\left(\mathbb{L}^{2} ; \mathbb{L}^{2}\right)}^{2} d s\right] \\
& +\mathbb{E}\left[\int_{0}^{t \wedge \tau_{N}} \int_{Z}\left\|H^{n}\left(u^{n}(s), z\right)\right\|_{\mathbb{L}^{2}}^{2} \lambda(d z) d s\right]+\mathbb{E}\left[\left\|u_{0}^{n}\right\|_{\mathbb{L}^{2}}^{2}+\left\|\hat{z}_{0}^{n}\right\|_{L^{2}}^{2}\right] \\
\leq & C \mathbb{E}\left[\int_{0}^{t \wedge \tau_{N}}\left(\left\|u^{n}(s)\right\|_{\mathbb{L}^{2}}^{2}+\left\|\hat{z}^{n}(s)\right\|_{L^{2}}^{2}+\left\|w^{0}(s)\right\|_{\mathbb{L}^{4}}^{4}\right) d s\right] \\
& +\mathbb{E}\left[\int_{0}^{t \wedge \tau_{N}}\|f(s)\|_{\mathbb{L}^{2}}^{2} d s\right]+K \mathbb{E}\left[\int_{0}^{t \wedge \tau_{N}}\left(1+\left\|u^{n}(s)\right\|_{\mathbb{L}^{2}}^{2}\right) d s\right] \\
& +\mathbb{E}\left[\left\|u_{0}^{n}\right\|_{\mathbb{L}^{2}}^{2}+\left\|\hat{z}_{0}^{n}\right\|_{L^{2}}^{2}\right] \\
\leq & \mathbb{E}\left[\left\|u_{0}\right\|_{\mathbb{L}^{2}}^{2}+\left\|\hat{z}_{0}\right\|_{L^{2}}^{2}+C \int_{0}^{t \wedge \tau_{N}}\left(\left\|w^{0}(s)\right\|_{\mathbb{L}^{4}}^{4}+\|f(s)\|_{\mathbb{L}^{2}}^{2}\right) d s+K\left(t \wedge \tau_{N}\right)\right] \\
& +C \mathbb{E}\left[\int_{0}^{t \wedge \tau_{N}}\left(\left\|u^{n}(s)\right\|_{\mathbb{L}^{2}}^{2}+\left\|\hat{z}^{n}(s)\right\|_{L^{2}}^{2}\right) d s\right] .
\end{aligned}
$$

Now Gronwall's inequality yields

$$
\begin{aligned}
& \mathbb{E}\left[\left\|u^{n}\left(t \wedge \tau_{N}\right)\right\|_{\mathbb{L}^{2}}^{2}+\left\|\hat{z}^{n}\left(t \wedge \tau_{N}\right)\right\|_{L^{2}}^{2}\right]+\alpha \mathbb{E}\left[\int_{0}^{t \wedge \tau_{N}}\left\|u^{n}(s)\right\|_{\mathbb{H}_{0}^{1}}^{2} d s\right] \\
& \leq C \mathbb{E}\left[\left\|u_{0}\right\|_{\mathbb{L}^{2}}^{2}+\left\|\hat{z}_{0}\right\|_{L^{2}}^{2}+C \int_{0}^{t \wedge \tau_{N}}\left(\left\|w^{0}(s)\right\|_{\mathbb{L}^{4}}^{4}+\|f(s)\|_{\mathbb{L}^{2}}^{2}\right) d s+K\left(t \wedge \tau_{N}\right)\right] e^{C\left(t \wedge \tau_{N}\right)} .
\end{aligned}
$$

Finally, taking limit as $N \rightarrow \infty$ we have

$$
\begin{aligned}
& \mathbb{E}\left[\left\|u^{n}(t)\right\|_{\mathbb{L}^{2}}^{2}+\left\|\hat{z}^{n}(t)\right\|_{L^{2}}^{2}\right]+\alpha \mathbb{E}\left[\int_{0}^{t}\left\|u^{n}(s)\right\|_{\mathbb{H}_{0}^{1}}^{2} d s\right] \\
& \leq C \mathbb{E}\left[\left\|u_{0}\right\|_{\mathbb{L}^{2}}^{2}+\left\|\hat{z}_{0}\right\|_{L^{2}}^{2}+C \int_{0}^{t}\left(\left\|w^{0}(s)\right\|_{\mathbb{L}^{4}}^{4}+\|f(s)\|_{\mathbb{L}^{2}}^{2}\right) d s+K t\right] e^{C t} \\
& \leq C \mathbb{E}\left[\left\|u_{0}\right\|_{\mathbb{L}^{2}}^{2}+\left\|\hat{z}_{0}\right\|_{L^{2}}^{2}+C \int_{0}^{T}\left(\left\|w^{0}(s)\right\|_{\mathbb{L}^{4}}^{4}+\|f(s)\|_{\mathbb{L}^{2}}^{2}\right) d s+K T\right] e^{C T},
\end{aligned}
$$

which is the desired a-priori estimate (4.5).

Proceeding similarly, by taking supremum of equation (4.16) over time and then taking expectation we achieve

$$
\begin{aligned}
& \mathbb{E}\left[\sup _{0 \leq t \leq T \wedge \tau_{N}}\left(\left\|u^{n}(t)\right\|_{\mathbb{L}^{2}}^{2}+\left\|\hat{z}^{n}(t)\right\|_{L^{2}}^{2}\right)\right]+\alpha \mathbb{E}\left[\int_{0}^{T \wedge \tau_{N}}\left\|u^{n}(s)\right\|_{\mathbb{H}_{0}^{1}}^{2} d s\right] \\
& \leq C \mathbb{E}\left[\int_{0}^{T \wedge \tau_{N}}\left(\left\|u^{n}(s)\right\|_{\mathbb{L}^{2}}^{2}+\left\|\hat{z}^{n}(s)\right\|_{L^{2}}^{2}+\left\|w^{0}(s)\right\|_{\mathbb{L}^{4}}^{4}\right) d s\right] \\
& \quad+\mathbb{E}\left[\int_{0}^{T \wedge \tau_{N}}\|f(s)\|_{\mathbb{L}^{2}}^{2} d s\right]+K\left(T \wedge \tau_{N}\right)+K \mathbb{E}\left[\int_{0}^{T \wedge \tau_{N}}\left\|u^{n}(s)\right\|_{\mathbb{L}^{2}}^{2} d s\right]
\end{aligned}
$$




$$
\begin{aligned}
& +2 \mathbb{E}\left[\sup _{0 \leq t \leq T \wedge \tau_{N}}\left|\int_{0}^{t}\left(\sigma^{n}\left(s, u^{n}(s)\right) d W^{n}(s), u^{n}(s)\right)_{\mathbb{L}^{2}}\right|\right]+\mathbb{E}\left[\left\|u_{0}^{n}\right\|_{\mathbb{L}^{2}}^{2}+\left\|\hat{z}_{0}^{n}\right\|_{L^{2}}^{2}\right] \\
& +2 \mathbb{E}\left[\sup _{0 \leq t \leq T \wedge \tau_{N}}\left|\int_{0}^{t} \int_{Z}\left(H^{n}\left(u^{n}(s-), z\right), u^{n}(s-)\right)_{\mathbb{L}^{2}} \tilde{N}(d s, d z)\right|\right] .
\end{aligned}
$$

Using Burkholder-Davis-Gundy inequality, (4.18), Assumption 3.12 and Young's inequality we have

$$
\begin{aligned}
& 2 \mathbb{E}\left[\sup _{0 \leq t \leq T \wedge \tau_{N}}\left|\int_{0}^{t}\left(\sigma^{n}\left(s, u^{n}(s)\right) d W^{n}(s), u^{n}(s)\right)_{\mathbb{L}^{2}}\right|\right] \\
& \leq C_{3} \mathbb{E}\left[\left(\int_{0}^{T \wedge \tau_{N}}\left\|\sigma^{n}\left(s, u^{n}(s)\right)\right\|_{L_{Q}\left(\mathbb{L}^{2}, \mathbb{L}^{2}\right)}^{2}\left\|u^{n}(s)\right\|_{\mathbb{L}^{2}}^{2} d s\right)\right]^{1 / 2} \\
& \leq C_{3} \mathbb{E}\left[\left(\int_{0}^{T \wedge \tau_{N}}\left\|\sigma\left(s, u^{n}(s)\right)\right\|_{L_{Q}\left(\mathbb{L}^{2}, \mathbb{L}^{2}\right)}^{2}\left\|u^{n}(s)\right\|_{\mathbb{L}^{2}}^{2} d s\right)\right]^{1 / 2} \\
& \leq C_{3} K \mathbb{E}\left[\left(\int_{0}^{T \wedge \tau_{N}}\left(1+\left\|u^{n}(s)\right\|_{\mathbb{L}^{2}}^{2}\right)\left\|u^{n}(s)\right\|_{\mathbb{L}^{2}}^{2} d s\right)\right]^{1 / 2} \\
& \leq C_{3} K \mathbb{E}\left[\sup _{0 \leq s \leq T \wedge \tau_{N}}\left\|u^{n}(s)\right\|_{\mathbb{L}^{2}}^{2}\left(\int_{0}^{T \wedge \tau_{N}}\left(1+\left\|u^{n}(s)\right\|_{\mathbb{L}^{2}}^{2}\right) d s\right)\right]^{1 / 2} \\
& \leq \frac{1}{4} \mathbb{E}\left[\sup _{0 \leq s \leq T \wedge \tau_{N}}\left\|u^{n}(s)\right\|_{\mathbb{L}^{2}}^{2}\right]+\left(C_{3} K\right)^{2} \mathbb{E}\left[\int_{0}^{T \wedge \tau_{N}}\left\|u^{n}(s)\right\|_{\mathbb{L}^{2}}^{2} d s\right] \\
& +\left(C_{3} K\right)^{2}\left(T \wedge \tau_{N}\right) .
\end{aligned}
$$

Similarly, using Burkholder-Davis-Gundy inequality [see Ichikawa [22] ], (4.19), Assumption 3.12 and Young's inequality we have

$$
\begin{aligned}
& \left.2 \mathbb{E} \sup _{0 \leq t \leq T \wedge \tau_{N}}\left|\int_{0}^{t} \int_{Z}\left(H^{n}\left(u^{n}(s-), z\right), u^{n}(s-)\right)_{\mathbb{L}^{2}} \tilde{N}(d s, d z)\right|\right] \\
& \leq C_{4} \mathbb{E}\left[\left(\int_{0}^{T \wedge \tau_{N}} \int_{Z}\left|\left(H^{n}\left(u^{n}(s), z\right), u^{n}(s)\right)_{\mathbb{L}^{2}}\right|^{2} \lambda(d z) d s\right)\right]^{1 / 2} \\
& \leq C_{4} \mathbb{E}\left[\left(\int_{0}^{T \wedge \tau_{N}} \int_{Z}\left\|H^{n}\left(u^{n}(s), z\right)\right\|_{\mathbb{L}^{2}}^{2}\left\|u^{n}(s)\right\|_{\mathbb{L}^{2}}^{2} \lambda(d z) d s\right)\right]^{1 / 2} \\
& \leq C_{4} \mathbb{E}\left[\left(\int_{0}^{T \wedge \tau_{N}} \int_{Z}\left\|H\left(u^{n}(s), z\right)\right\|_{\mathbb{L}^{2}}^{2}\left\|u^{n}(s)\right\|_{\mathbb{L}^{2}}^{2} \lambda(d z) d s\right)\right]^{1 / 2} \\
& \leq C_{4} K \mathbb{E}\left[\sup _{0 \leq s \leq T \wedge \tau_{N}}\left\|u^{n}(s)\right\|_{\mathbb{L}^{2}}^{2}\left(\int_{0}^{T \wedge \tau_{N}}\left(1+\left\|u^{n}(s)\right\|_{\mathbb{L}^{2}}^{2}\right) d s\right)\right]^{1 / 2} \\
& \leq \frac{1}{4}\left[\sup _{0 \leq s \leq T \wedge \tau_{N}}\left\|u^{n}(s)\right\|_{\mathbb{L}^{2}}^{2}\right]+\left(C_{4} K\right)^{2} \mathbb{E}\left[\int_{0}^{T \wedge \tau_{N}}\left\|u^{n}(s)\right\|_{\mathbb{L}^{2}}^{2} d s\right] \\
& +\left(C_{4} K\right)^{2}\left(T \wedge \tau_{N}\right) .
\end{aligned}
$$

Substituting equations (4.21) and (4.22) in equation (4.20) and rearranging the terms we have

$$
\begin{aligned}
& \mathbb{E}\left[\sup _{0 \leq t \leq T \wedge \tau_{N}}\left(\left\|u^{n}(t)\right\|_{\mathbb{L}^{2}}^{2}+\left\|\hat{z}^{n}(t)\right\|_{L^{2}}^{2}\right)\right]+2 \alpha \mathbb{E}\left[\int_{0}^{T \wedge \tau_{N}}\left\|u^{n}(s)\right\|_{\mathbb{H}_{0}^{1}}^{2} d s\right] \\
& \leq C^{\prime} \mathbb{E}\left[\int_{0}^{T \wedge \tau_{N}} \sup _{0 \leq s \leq t}\left(\left\|u^{n}(s)\right\|_{\mathbb{L}^{2}}^{2}+\left\|\hat{z}^{n}(s)\right\|_{L^{2}}^{2}\right) d t\right]
\end{aligned}
$$




$$
\begin{aligned}
& +C \mathbb{E}\left[\int_{0}^{T \wedge \tau_{N}}\left\|w^{0}(s)\right\|_{\mathbb{L}^{4}}^{4} d s\right]+2 \mathbb{E}\left[\int_{0}^{T \wedge \tau_{N}}\|f(s)\|_{\mathbb{L}^{2}}^{2} d s\right] \\
& +C^{\prime \prime}\left(T \wedge \tau_{N}\right)+2 \mathbb{E}\left[\left\|u_{0}^{n}\right\|_{\mathbb{L}^{2}}^{2}+\left\|\hat{z}_{0}^{n}\right\|_{L^{2}}^{2}\right] \\
& \leq C^{\prime \prime \prime} \mathbb{E}\left[\left\|u_{0}\right\|_{\mathbb{L}^{2}}^{2}+\left\|\hat{z}_{0}\right\|_{L^{2}}^{2}+\int_{0}^{T \wedge \tau_{N}}\left(\left\|w^{0}(s)\right\|_{\mathbb{L}^{4}}^{4}+\|f(s)\|_{\mathbb{L}^{2}}^{2}\right) d s\right] \\
& +C^{\prime \prime}\left(T \wedge \tau_{N}\right)+C^{\prime} \mathbb{E}\left[\int_{0}^{T \wedge \tau_{N}} \sup _{0 \leq s \leq t}\left(\left\|u^{n}(s)\right\|_{\mathbb{L}^{2}}^{2}+\left\|\hat{z}^{n}(s)\right\|_{L^{2}}^{2}\right) d t\right],
\end{aligned}
$$

where $C^{\prime}=2\left[C+\left(C_{3} K\right)^{2}+\left(C_{4} K\right)^{2}+K\right], C^{\prime \prime}=2\left[\left(C_{3} K\right)^{2}+\left(C_{4} K\right)^{2}+K\right]$ and $C^{\prime \prime \prime}=\max \{C, 2\}$. Now Gronwall's inequality yields

$$
\begin{aligned}
& \mathbb{E}\left[\sup _{0 \leq t \leq T \wedge \tau_{N}}\left(\left\|u^{n}(t)\right\|_{\mathbb{L}^{2}}^{2}+\left\|\hat{z}^{n}(t)\right\|_{L^{2}}^{2}\right)\right]+2 \alpha \mathbb{E}\left[\int_{0}^{T \wedge \tau_{N}}\left\|u^{n}(s)\right\|_{\mathbb{H}_{0}^{1}}^{2} d s\right] \\
& \leq C^{\prime \prime \prime} \mathbb{E}\left[\left\|u_{0}\right\|_{\mathbb{L}^{2}}^{2}+\left\|\hat{z}_{0}\right\|_{L^{2}}^{2}+\int_{0}^{T \wedge \tau_{N}}\left(\left\|w^{0}(s)\right\|_{\mathbb{L}^{4}}^{4}+\|f(s)\|_{\mathbb{L}^{2}}^{2}\right) d s+C^{\prime \prime}\left(T \wedge \tau_{N}\right)\right] e^{C^{\prime}\left(T \wedge \tau_{N}\right)} .
\end{aligned}
$$

Taking limit as $N \rightarrow \infty$ we infer that

$$
\begin{aligned}
& \mathbb{E}\left[\sup _{0 \leq t \leq T}\left(\left\|u^{n}(t)\right\|_{\mathbb{L}^{2}}^{2}+\left\|\hat{z}^{n}(t)\right\|_{L^{2}}^{2}\right)\right]+2 \alpha \mathbb{E}\left[\int_{0}^{T}\left\|u^{n}(s)\right\|_{\mathbb{H}_{0}^{1}}^{2} d s\right] \\
& \leq C^{\prime \prime \prime} \mathbb{E}\left[\left\|u_{0}\right\|_{\mathbb{L}^{2}}^{2}+\left\|\hat{z}_{0}\right\|_{L^{2}}^{2}+\int_{0}^{T}\left(\left\|w^{0}(s)\right\|_{\mathbb{L}^{4}}^{4}+\|f(s)\|_{\mathbb{L}^{2}}^{2}\right) d s+C^{\prime \prime} T\right] e^{C^{\prime} T} .
\end{aligned}
$$

which is the desired a-priori estimate (4.6).

4.1. $p^{\text {th }}$ Moment Estimate. Let $2 \leq p<\infty$. We assume that $\sigma$ and $H$ satisfy Assumption 3.12 provided in Subsection 3.12. In addition, we assume

H.4 for all $t \in(0, T)$, there exists a positive constant $M$ such that for all $2 \leq p<\infty$ and $u \in \mathbb{L}^{2}(\mathcal{O})$

$$
\int_{Z}\|H(u, z)\|_{\mathbb{L}^{2}}^{p} \lambda(d z) \leq M\left(1+\|u\|_{\mathbb{L}^{2}}^{p}\right) .
$$

Then we have the following result.

Proposition 4.4. Let $2 \leq p<\infty, p^{*}=2 p$ and

$$
\left\{\begin{array}{l}
w^{0} \in L^{p^{*}}\left(\Omega ; L^{p^{*}}\left(0, T ; \mathbb{L}^{p^{*}}(\mathcal{O})\right)\right), f \in L^{p}\left(\Omega ; L^{p}\left(0, T ; \mathbb{L}^{2}(\mathcal{O})\right)\right), \\
\sigma \in C\left([0, T] \times \mathbb{H}_{0}^{1}(\mathcal{O}) ; L_{Q}\left(\mathbb{L}^{2}, \mathbb{L}^{2}\right)\right), H \in \mathbb{H}_{\lambda}^{p}\left([0, T] \times Z ; \mathbb{L}^{2}(\mathcal{O})\right), \\
u_{0} \in L^{p}\left(\Omega ; \mathbb{L}^{2}(\mathcal{O})\right), \hat{z}_{0} \in L^{p}\left(\Omega ; L^{2}(\mathcal{O})\right) .
\end{array}\right.
$$

If $\left(u^{n}(t), \hat{z}^{n}(t)\right)$ denotes the unique strong solution of the system (4.1)-(4.3), then we have the following a-priori estimate:

$$
\mathbb{E}\left[\sup _{0 \leq t \leq T}\left(\left\|u^{n}(t)\right\|_{\mathbb{L}^{2}}^{p}+\left\|\hat{z}^{n}(t)\right\|_{L^{2}}^{p}\right)\right]+\alpha p \mathbb{E}\left[\int_{0}^{T}\left\|u^{n}(t)\right\|_{\mathbb{L}^{2}}^{p-2}\left\|u^{n}(t)\right\|_{\mathbb{H}_{0}^{1}}^{2} d t\right] \leq C_{1(p)},
$$

where the constant $C_{1(p)}$ depends on the coefficients $\alpha, g, M, \mu$ and the norms $\|f\|_{L^{p}\left(\Omega ; L^{p}\left(0, T ; \mathbb{L}^{2}\right)\right)}$, $\left\|w^{0}\right\|_{L^{p^{*}}\left(\Omega ; L^{p^{*}}\left(0, T ; \mathbb{L}^{p^{*}}\right)\right)},\left\|u_{0}\right\|_{L^{p}\left(\Omega ; \mathbb{L}^{2}\right)},\left\|\hat{z}_{0}\right\|_{L^{p}\left(\Omega ; L^{2}\right)}$ and $T$.

The Proposition can be proved using the same ideas exploited in Proposition 4.3 . However, we provide certain steps similar to (4.9) which clarifies regularity of $w^{0} \in L^{p^{*}}\left(\Omega ; L^{p^{*}}\left(0, T ; \mathbb{L}^{p^{*}}(\mathcal{O})\right)\right)$. Using the definition of the operator $B(\cdot)$ and Lemma 3.2 we have

$$
\left(B\left(u^{n}(t)\right), u^{n}(t)\right)_{\mathbb{L}^{2}}\left|u^{n}(t)\right|^{p-2} \geq-\int_{\mathcal{O}} \gamma(x)\left|w^{0}(t)\right|^{2}\left|u^{n}(t)\right|^{p-1} d x
$$




$$
\begin{aligned}
& \geq-\frac{r}{\epsilon}\left(\int_{\mathcal{O}}\left|w^{0}(t)\right|^{2 p} d x\right)^{\frac{1}{p}}\left(\int_{\mathcal{O}}\left|u^{n}(t)\right|^{p} d x\right)^{\frac{p-1}{p}} \\
& \geq-\frac{r}{\epsilon}\left[\left\|u^{n}(t)\right\|_{\mathbb{L}^{p}}^{p-1}\left\|w^{0}(t)\right\|_{\mathbb{L}^{2 p}}^{2}\right] \\
& \geq-\frac{r}{\epsilon}\left[\frac{p-1}{p}\left\|u^{n}(t)\right\|_{\mathbb{L}^{p}}^{p}+\frac{1}{p}\left\|w^{0}(t)\right\|_{\mathbb{L}^{2 p}}^{2 p}\right]
\end{aligned}
$$

\subsection{Existence Result.}

Definition 4.5. A path-wise strong solution $(u, \hat{z})$ is defined on a given filtered probability space $\left(\Omega, \mathcal{F}, \mathcal{F}_{t}, P\right)$ as a $\left(L^{\infty}\left(0, T ; \mathbb{L}^{2}(\mathcal{O})\right) \cap L^{2}\left(0, T ; \mathbb{H}_{0}^{1}(\mathcal{O})\right)\right) \times C\left([0, T] ; L^{2}(\mathcal{O})\right)$ valued function which satisfy the stochastic tide equations (1.1)-(1.3) in the weak sense and also the energy inequalities in Proposition 4.3 .

Proof of Theorem 1.1. Step I : Weak convergent subsequences

Recall that the Galerkin approximations $\left(u^{n}, \hat{z}^{n}\right)$ satisfy the stochastic differential equations

$$
\begin{aligned}
d u^{n}(t)+F\left(u^{n}(t)\right) d t+g \nabla \hat{z}^{n}(t) d t & =\sigma^{n}\left(t, u^{n}(t)\right) d W^{n}(t) \\
& +\int_{Z} H^{n}\left(u^{n}(t-), z\right) \tilde{N}(d t, d z), \\
d \hat{z}^{n}(t)+\operatorname{Div}\left(h u^{n}(t)\right) d t=0, &
\end{aligned}
$$

where $F\left(u^{n}\right)=A u^{n}+B\left(u^{n}\right)-f$. Then using the a-priori estimates (4.5)-(4.6), it follows from the Banach-Alaoglu theorem that along a subsequence of $\left(u^{n}, \hat{z}^{n}\right)$, still denoted by $\left(u^{n}, \hat{z}^{n}\right)$, we obtain the following limits:

$$
\begin{aligned}
& u^{n} \rightarrow u \text { weakly star in } L^{2}\left(\Omega ; L^{\infty}\left(0, T, \mathbb{L}^{2}(\mathcal{O})\right) \cap L^{2}\left(0, T ; \mathbb{H}_{0}^{1}(\mathcal{O})\right)\right), \\
& u^{n}(T) \rightarrow \eta \text { weakly in } L^{2}\left(\Omega ; \mathbb{L}^{2}(\mathcal{O})\right) \\
& \hat{z}^{n} \rightarrow \hat{z} \text { weakly in } L^{2}\left(\Omega ; L^{2}\left(0, T ; L^{2}(\mathcal{O})\right)\right) \\
& F\left(u^{n}\right) \rightarrow F_{0} \text { weakly in } L^{2}\left(\Omega ; L^{2}\left(0, T ; \mathbb{H}^{-1}(\mathcal{O})\right)\right) \\
& \sigma^{n}\left(\cdot, u^{n}\right) \rightarrow \sigma_{0} \text { weakly in } L^{2}\left(\Omega ; L^{2}\left(0, T ; L_{Q}\left(\mathbb{L}^{2}, \mathbb{L}^{2}\right)\right)\right) \\
& H^{n}\left(u^{n}, \cdot\right) \rightarrow H_{0} \text { weakly in } \mathbb{H}_{\lambda}^{2}\left([0, T] \times Z ; \mathbb{L}^{2}\right)
\end{aligned}
$$

Note that boundedness of $F\left(u^{n}\right)$ follows from the bounds of the operators $A$ and $B$, and convergences of $\sigma^{n}$ and $H^{n}$ follow from their linear growth property and the uniform bound of $u^{n}$ (in $n$ ) in $L^{2}\left(\Omega ; L^{2}\left(0, T ; \mathbb{H}_{0}^{1}(\mathcal{O})\right)\right)$.

Claim 1. $u$ satisfies the stochastic differential equation

$$
d u(t)+F_{0}(t) d t+g \nabla \hat{z}(t) d t=\sigma_{0}(t) d W(t)+\int_{Z} H_{0}(t, z) \tilde{N}(d t, d z),
$$

weakly in $L^{2}\left(\Omega ; L^{2}\left(0, T ; \mathbb{H}^{-1}(\mathcal{O})\right)\right)$.

\section{Proof of Claim 1.}

Our proof is based on Theorem 4.4 of Brzeźniak et al. 9] and Theorem 3.2 of Suvinthra et al. [47. Let us consider the function $\psi(t) \in H^{1}(-\delta, T+\delta)$ with $\psi(0)=1$. Let $\left\{e_{j}\right\}_{j \in \mathbb{N}}$ be an orthonormal basis in $\mathbb{H}_{0}^{1}(\mathcal{O})$. We define $e_{j}(t)=\psi(t) e_{j}$. Applying Itô formula to the function $\left(u^{n}(t), e_{j}(t)\right)_{\mathbb{L}^{2}}$ we have

$$
\begin{aligned}
& \left(u^{n}(T), e_{j}(T)\right)_{\mathbb{L}^{2}}-\int_{0}^{T}\left(u^{n}(s), \frac{d e_{j}(s)}{d s}\right)_{\mathbb{L}^{2}} d s+\int_{0}^{T}\left\langle F\left(u^{n}(s)\right)+g \nabla \hat{z}^{n}(s), e_{j}(s)\right\rangle d s \\
& =\left(u^{n}(0), e_{j}\right)_{\mathbb{L}^{2}}+\int_{0}^{T}\left(\sigma^{n}\left(s, u^{n}(s)\right) d W^{n}(s), e_{j}(s)\right)_{\mathbb{L}^{2}}+\int_{0}^{T} \int_{Z}\left(H^{n}\left(u^{n}(s-), z\right), e_{j}(s)\right)_{\mathbb{L}^{2}} \tilde{N}(d s, d z)
\end{aligned}
$$


We now fix the integer $j$. Let $\mathcal{P}_{T}^{1}$ denote the class of all predictable processes with values in $L^{2}\left(\Omega \times[0, T], L_{Q}\left(\mathbb{L}^{2}, \mathbb{L}^{2}\right)\right)$ with the inner product defined by $(\xi, \eta)_{\mathcal{P}_{T}^{1}}=\mathbb{E}\left[\int_{0}^{T} \operatorname{Tr}\left(\xi(t) Q \eta^{\star}(t)\right) d t\right]$ for all $\xi, \eta \in \mathcal{P}_{T}^{1}$. Define $J_{1}: \mathcal{P}_{T}^{1} \rightarrow L^{2}(\Omega \times[0, T])$ by $J_{1}\left(G_{1}\right)=\int_{0}^{t}\left(G_{1}(s) d W(s), e_{j}(s)\right)_{\mathbb{L}^{2}}$. Then $J_{1}$ is linear and continuous. Therefore, in view of weak convergence [as in Chow [15], Chapter 6.7, Proof of Theorem 7.5] of $\sigma^{n}\left(\cdot, u^{n}(\cdot)\right)$ to $\sigma_{0}(\cdot)$, we have

$$
J_{1}\left(\sigma^{n}\left(t, u^{n}(t)\right)\right)=\int_{0}^{T}\left(\sigma^{n}\left(s, u^{n}(s)\right) d W^{n}(s), e_{j}(s)\right)_{\mathbb{L}^{2}} \rightarrow \int_{0}^{t}\left(\sigma_{0}(s) d W(s), e_{j}(s)\right)_{\mathbb{L}^{2}} \quad \text { as } \quad n \rightarrow \infty .
$$

Again, let $\mathcal{P}_{T}^{2}$ be the class of all predictable process with values in $L^{2}\left(\Omega \times[0, T], \mathbb{H}_{\lambda}^{2}([0, T] \times\right.$ $\left.Z ; \mathbb{L}^{2}(\mathcal{O})\right)$ ) with the inner product defined by $(\zeta, \gamma)_{\mathcal{P}_{T}^{2}}=\mathbb{E}\left[\int_{0}^{T} \int_{Z}(\zeta(t), \gamma(t))_{\mathbb{L}^{2}} \lambda(d z) d t\right]$. Define $J_{2}: \mathcal{P}_{T}^{2} \rightarrow L^{2}(\Omega \times[0, T])$ by $J_{2}\left(G_{2}\right)=\int_{0}^{t} \int_{Z}\left(G_{2}(s, z), e_{j}(s)\right)_{\mathbb{L}^{2}} \tilde{N}(d s, d z)$. Then $J_{2}$ is linear and continuous (infact an isometry), hence it is continuous with respect to the weak topologies, [see Theorem 4.4 of Brzeźniak et al. [9] and references therein]. Again in view of weak convergence of $H^{n}\left(u^{n}(\cdot), \cdot\right)$ to $H_{0}$ we have,

$J_{2}\left(H^{n}\left(u^{n}(t), z\right)\right)=\int_{0}^{t} \int_{Z}\left(H^{n}\left(u^{n}(s-), z\right), e_{j}(s)\right)_{\mathbb{L}^{2}} \tilde{N}(d s, d z) \rightarrow \int_{0}^{t} \int_{Z}\left(H_{0}(s, z), e_{j}(s)\right)_{\mathbb{L}^{2}} \tilde{N}(d s, d z)$, as $n \rightarrow \infty$. Passing to the limit in (4.34) using weak convergence we have

$$
\begin{aligned}
& \left(\eta, e_{j}\right)_{\mathbb{L}^{2}} \psi(T)-\int_{0}^{T}\left(u(s), \frac{d \psi(s)}{d s} e_{j}\right)_{\mathbb{L}^{2}} d s+\int_{0}^{T}\left\langle F_{0}(s)+g \nabla \hat{z}(s), e_{j}\right\rangle d s \\
& \left.=\left(u(0), e_{j}\right)_{\mathbb{L}^{2}}+\int_{0}^{T}\left(\sigma_{0}(s) d W(s), \psi(s) e_{j}\right)_{\mathbb{L}^{2}}+\int_{0}^{T} \int_{Z}\left(H_{0}(s, z), z\right), \psi(s) e_{j}\right)_{\mathbb{L}^{2}} \tilde{N}(d s, d z) .
\end{aligned}
$$

We now choose a subsequence of functions $\left\{\psi_{k}\right\} \subset H^{1}(-\delta, T+\delta)$ such that $\psi_{k}(0)=1, k \in \mathbb{N}$, and letting $k \rightarrow \infty, \psi_{k}(s)$ converges to the Heaviside function $H(t-s)$ which is one for $s \leq t$ and zero otherwise. Replacing $\psi_{k}$ instead of $\psi$ as $k \rightarrow \infty$ we have,

$$
\begin{aligned}
& \left(u(t), e_{j}\right)_{\mathbb{L}^{2}}+\int_{0}^{t}\left\langle F_{0}(s)+g \nabla \hat{z}(s), e_{j}\right\rangle d s \\
& \left.\quad=\left(u(0), e_{j}\right)_{\mathbb{L}^{2}}+\int_{0}^{t}\left(\sigma_{0}(s) d W(s), e_{j}\right)_{\mathbb{L}^{2}}+\int_{0}^{t} \int_{Z}\left(H_{0}(s, z), z\right), e_{j}\right)_{\mathbb{L}^{2}} \tilde{N}(d s, d z)
\end{aligned}
$$

with $\left(u(T), e_{j}\right)_{\mathbb{L}^{2}}=\left(\eta, e_{j}\right)_{\mathbb{L}^{2}} ; j=1, \ldots$ This gives

$$
u(t)+\int_{0}^{t}\left(F_{0}(s)+g \nabla \hat{z}(s)\right) d s=u(0)+\int_{0}^{t} \sigma_{0}(s) d W(s)+\int_{0}^{t} \int_{Z} H_{0}(s, z) \tilde{N}(d s, d z),
$$

with $u(T)=\eta$. This proves the claim. Hence $u$ satisfies 4.33 weakly in $L^{2}\left(\Omega ; L^{2}\left(0, T ; \mathbb{H}^{-1}(\mathcal{O})\right)\right)$ and $\hat{z}$ satisfies

$$
d \hat{z}(t)+\operatorname{Div}(h u(t)) d t=0
$$

weakly in $L^{2}\left(\Omega ; L^{2}\left(0, T ; L^{2}(\mathcal{O})\right)\right)$.

\section{Step II : Energy inequality of weak limits}

Let us take $\tilde{\Lambda}:=2 \Lambda_{1}+L$ where $\Lambda_{1}$ is given in Remark 3.15. Let us apply Itô's lemma to the process $e^{-\tilde{\Lambda} t}\|\sqrt{h} u(t)\|_{\mathbb{L}^{2}}^{2}$ to get

$$
\begin{aligned}
& e^{-\tilde{\Lambda} T}\|\sqrt{h} u(T)\|_{\mathbb{L}^{2}}^{2}-\|\sqrt{h} u(0)\|_{\mathbb{L}^{2}}^{2}+e^{-\tilde{\Lambda} T} g\|\hat{z}(t)\|_{L^{2}}^{2}-g\|\hat{z}(0)\|_{L^{2}}^{2} \\
& =-\tilde{\Lambda} \int_{0}^{T} e^{-\tilde{\Lambda} t} g\|\hat{z}(t)\|_{\mathbb{L}^{2}}^{2} d t-\tilde{\Lambda} \int_{0}^{T} e^{-\tilde{\Lambda} t}\|\sqrt{h} u(t)\|_{\mathbb{L}^{2}}^{2} d t
\end{aligned}
$$




$$
\begin{aligned}
& -\int_{0}^{T} e^{-\tilde{\Lambda} t}\left\langle 2 F_{0}(t), h u(t)\right\rangle d t+2 \int_{0}^{T} e^{-\tilde{\Lambda} t}\left(\sigma_{0}(t) d W(t), h u(t)\right)_{\mathbb{L}^{2}} \\
& +\int_{0}^{T} e^{-\tilde{\Lambda} t}\left\|\sqrt{h} \sigma_{0}(t)\right\|_{L_{Q}\left(\mathbb{L}^{2}, \mathbb{L}^{2}\right)}^{2} d t+2 \int_{0}^{T} e^{-\tilde{\Lambda} t} \int_{Z}\left(H_{0}(t, z), h u(t-)\right)_{\mathbb{L}^{2}} \tilde{N}(d t, d z) \\
& +\int_{0}^{T} e^{-\tilde{\Lambda} t} \int_{Z}\left\|\sqrt{h} H_{0}(t, z)\right\|_{\mathbb{L}^{2}}^{2} N(d t, d z) .
\end{aligned}
$$

Similarly applying Itô's lemma to the process $e^{-\tilde{\Lambda} t}\left\|\sqrt{h} u^{n}(t)\right\|_{\mathbb{L}^{2}}^{2}$ and then taking expectation we obtain

$$
\begin{aligned}
& \mathbb{E}\left[e^{-\tilde{\Lambda} T}\left\|\sqrt{h} u^{n}(T)\right\|_{\mathbb{L}^{2}}^{2}+e^{-\tilde{\Lambda} T} g\left\|\hat{z}^{n}(T)\right\|_{L^{2}}^{2}-\left\|\sqrt{h} u^{n}(0)\right\|_{\mathbb{L}^{2}}^{2}-g\left\|\hat{z}^{n}(0)\right\|_{L^{2}}^{2}\right] \\
& =-\mathbb{E}\left[\int_{0}^{T} \tilde{\Lambda} e^{-\tilde{\Lambda} t} g\left\|\hat{z}^{n}(t)\right\|_{\mathbb{L}^{2}}^{2} d t\right]-\mathbb{E}\left[\int_{0}^{T} \tilde{\Lambda} e^{-\tilde{\Lambda} t}\left\|\sqrt{h} u^{n}(t)\right\|_{\mathbb{L}^{2}}^{2} d t\right] \\
& \quad-2 \mathbb{E}\left[\int_{0}^{T} e^{-\tilde{\Lambda} t}\left\langle F\left(u^{n}(t)\right), h u^{n}(t)\right\rangle d t\right]+\mathbb{E}\left[\int_{0}^{T} e^{-\tilde{\Lambda} t}\left\|\sqrt{h} \sigma^{n}\left(t, u^{n}(t)\right)\right\|_{L_{Q}\left(\mathbb{L}^{2}, \mathbb{L}^{2}\right)}^{2} d t\right] \\
& \quad+\mathbb{E}\left[\int_{0}^{T} 2 e^{-\tilde{\Lambda} t}\left(\sigma^{n}\left(t, u^{n}(t)\right) d W^{n}(t), h u^{n}(t)\right)_{\mathbb{L}^{2}}\right] \\
& \quad+\mathbb{E}\left[\int_{0}^{T} e^{-\tilde{\Lambda} t} \int_{Z}\left\|\sqrt{h} H^{n}\left(u^{n}(t-), z\right)\right\|_{\mathbb{L}^{2}}^{2} N(d t, d z)\right] \\
& \quad+\mathbb{E}\left[\int_{0}^{T} 2 e^{-\tilde{\Lambda} t} \int_{Z}\left(H^{n}\left(u^{n}(t-), z\right), h u^{n}(t-)\right)_{\mathbb{L}^{2}} \tilde{N}(d t, d z)\right] .
\end{aligned}
$$

Using the facts that

$$
\int_{0}^{T} 2 e^{-\tilde{\Lambda} t}\left(\sigma^{n}\left(t, u^{n}(t)\right) d W^{n}(t), h u^{n}(t)\right)_{\mathbb{L}^{2}}
$$

and

$$
\int_{0}^{T} 2 e^{-\tilde{\Lambda} t} \int_{Z}\left(H^{n}\left(u^{n}(t-), z\right), h u^{n}(t-)\right)_{\mathbb{L}^{2}} \tilde{N}(d t, d z),
$$

are local martingales with zero averages, and the expectation of quadratic variation process and that of Meyer process are same, i.e.,

$$
\begin{aligned}
& \mathbb{E}\left[\int_{0}^{T} e^{-\tilde{\Lambda} t} \int_{Z}\left\|\sqrt{h} H^{n}\left(u^{n}(t-), z\right)\right\|_{\mathbb{L}^{2}}^{2} N(d t, d z)\right] \\
& =\mathbb{E}\left[\int_{0}^{T} e^{-\tilde{\Lambda} t} \int_{Z}\left\|\sqrt{h} H^{n}\left(u^{n}(t), z\right)\right\|_{\mathbb{L}^{2}}^{2} \lambda(d z) d t\right]
\end{aligned}
$$

we have

$$
\begin{aligned}
& \mathbb{E}\left[e^{-\tilde{\Lambda} T}\left\|\sqrt{h} u^{n}(T)\right\|_{\mathbb{L}^{2}}^{2}+e^{-\tilde{\Lambda} T} g\left\|\hat{z}^{n}(T)\right\|_{L^{2}}^{2}-\left\|\sqrt{h} u^{n}(0)\right\|_{\mathbb{L}^{2}}^{2}-g\left\|\hat{z}^{n}(0)\right\|_{L^{2}}^{2}\right] \\
& =-\mathbb{E}\left[\int_{0}^{T} \tilde{\Lambda} e^{-\tilde{\Lambda} t} g\left\|\hat{z}^{n}(t)\right\|_{\mathbb{L}^{2}}^{2} d t\right]-\mathbb{E}\left[\int_{0}^{T} \tilde{\Lambda} e^{-\tilde{\Lambda} t}\left\|\sqrt{h} u^{n}(t)\right\|_{\mathbb{L}^{2}}^{2} d t\right] \\
& \quad-2 \mathbb{E}\left[\int_{0}^{T} e^{-\tilde{\Lambda} t}\left\langle F\left(u^{n}(t)\right), h u^{n}(t)\right\rangle d t\right]+\mathbb{E}\left[\int_{0}^{T} e^{-\tilde{\Lambda} t}\left\|\sqrt{h} \sigma^{n}\left(t, u^{n}(t)\right)\right\|_{L_{Q}\left(\mathbb{L}^{2}, \mathbb{L}^{2}\right)}^{2} d t\right] \\
& \quad+\mathbb{E}\left[\int_{0}^{T} e^{-\tilde{\Lambda} t} \int_{Z}\left\|\sqrt{h} H^{n}\left(u^{n}(t), z\right)\right\|_{\mathbb{L}^{2}}^{2} \lambda(d z) d t\right] .
\end{aligned}
$$

Using the lower semi-continuity property of the $\mathbb{L}^{2}$-norm, strong convergence of the initial data and applying Itô's lemma to the process $e^{-\tilde{\Lambda} t}\|\sqrt{h} u(t)\|_{\mathbb{L}^{2}}^{2}($ see (4.36) $)$, we infer that

$$
\begin{aligned}
& \liminf _{n}\left\{-\mathbb{E}\left[\int_{0}^{T} \tilde{\Lambda} e^{-\tilde{\Lambda} t}\left\|\sqrt{h} u^{n}(t)\right\|_{\mathbb{L}^{2}}^{2} d t\right]-2 \mathbb{E}\left[\int_{0}^{T} e^{-\tilde{\Lambda} t}\left\langle F\left(u^{n}(t)\right), h u^{n}(t)\right\rangle d t\right]\right. \\
& +\mathbb{E}\left[\int_{0}^{T} e^{-\tilde{\Lambda} t}\left\|\sqrt{h} \sigma^{n}\left(t, u^{n}(t)\right)\right\|_{L_{Q}\left(\mathbb{L}^{2}, \mathbb{L}^{2}\right)}^{2} d t\right]
\end{aligned}
$$




$$
\begin{aligned}
& \left.+\mathbb{E}\left[\int_{0}^{T} e^{-\tilde{\Lambda} t} \int_{Z}\left\|\sqrt{h} H^{n}\left(u^{n}(t), z\right)\right\|_{\mathbb{L}^{2}}^{2} \lambda(d z) d t\right]\right\} \\
= & \liminf _{n}\left\{\mathbb { E } \left[e^{-\tilde{\Lambda} T}\left\|\sqrt{h} u^{n}(T)\right\|_{\mathbb{L}^{2}}^{2}+e^{-\tilde{\Lambda} T} g\left\|\hat{z}^{n}(T)\right\|_{L^{2}}^{2}-\left\|\sqrt{h} u^{n}(0)\right\|_{\mathbb{L}^{2}}^{2}\right.\right. \\
& \left.\left.-g\left\|\hat{z}^{n}(0)\right\|_{L^{2}}^{2}\right]+\mathbb{E}\left[\int_{0}^{T} \tilde{\Lambda} e^{-\tilde{\Lambda} t} g\left\|\hat{z}^{n}(t)\right\|_{\mathbb{L}^{2}}^{2} d t\right]\right\} \\
\geq & \mathbb{E}\left[e^{-\tilde{\Lambda} T}\|\sqrt{h} u(T)\|_{\mathbb{L}^{2}}^{2}+e^{-\tilde{\Lambda} T} g\|\hat{z}(T)\|_{L^{2}}^{2}-\|\sqrt{h} u(0)\|_{\mathbb{L}^{2}}^{2}-g\|\hat{z}(0)\|_{L^{2}}^{2}\right] \\
& +\mathbb{E}\left[\int_{0}^{T} \tilde{\Lambda} e^{-\tilde{\Lambda} t} g\|\hat{z}(t)\|_{\mathbb{L}^{2}}^{2} d t\right] \\
= & -\mathbb{E}\left[\int_{0}^{T} \tilde{\Lambda} e^{-\tilde{\Lambda} t}\|\sqrt{h} u(t)\|_{\mathbb{L}^{2}}^{2} d t\right]-2 \mathbb{E}\left[\int_{0}^{T} e^{-\tilde{\Lambda} t}\left\langle F_{0}(t), h u(t)\right\rangle d t\right] \\
& +\mathbb{E}\left[\int_{0}^{T} e^{-\tilde{\Lambda} t}\left\|\sqrt{h} \sigma_{0}(t)\right\|_{L_{Q}\left(\mathbb{L}^{2}, \mathbb{L}^{2}\right)}^{2} d t\right]+\mathbb{E}\left[\int_{0}^{T} e^{-\tilde{\Lambda} t} \int_{Z}\left\|\sqrt{h} H_{0}(t, z)\right\|_{\mathbb{L}^{2}}^{2} \lambda(d z) d t\right] .
\end{aligned}
$$

\section{Step III : Consequences of weak convergence}

Claim 2. For any $v \in L^{2}\left(\Omega ; L^{\infty}\left(0, T ; \mathbb{L}^{2}(\mathcal{O})\right) \cap L^{2}\left(0, T ; \mathbb{H}_{0}^{1}(\mathcal{O})\right)\right)$, using the weak convergences (4.27) -(4.32), we have the following:

$$
\begin{aligned}
& \text { (i) } \mathbb{E}\left[\int_{0}^{T} e^{-\tilde{\Lambda} t}\left\langle F(v(t)), h u^{n}(t)-h v(t)\right\rangle d t\right] \\
& \rightarrow \mathbb{E}\left[\int_{0}^{T} e^{-\tilde{\Lambda} t}\langle F(v(t)), h u(t)-h v(t)\rangle d t\right] \quad \text { as } n \rightarrow \infty . \\
& \text { (ii) } \mathbb{E}\left[\int_{0}^{T} e^{-\tilde{\Lambda} t}\left(\sigma^{n}\left(t, u^{n}(t)\right), \sigma^{n}(t, v(t))\right)_{L_{Q}\left(\mathbb{L}^{2}, \mathbb{L}^{2}\right)} d t\right] \\
& \rightarrow \mathbb{E}\left[\int_{0}^{T} e^{-\tilde{\Lambda} t}\left(\sigma_{0}(t), \sigma(t, v(t))\right)_{L_{Q}\left(\mathbb{L}^{2}, \mathbb{L}^{2}\right)} d t\right] \quad \text { as } \quad n \rightarrow \infty . \\
& \text { (iii) } \mathbb{E}\left[\int_{0}^{T} e^{-\tilde{\Lambda} t} \int_{Z}\left(H^{n}\left(u^{n}(t), z\right), H^{n}(v(t), z)\right)_{\mathbb{L}^{2}} \lambda(d z) d t\right] \\
& \rightarrow \mathbb{E}\left[\int_{0}^{T} e^{-\tilde{\Lambda} t} \int_{Z}\left(H_{0}(t, z), H(v(t), z)\right)_{\mathbb{L}^{2}} \lambda(d z) d t\right] \quad \text { as } \quad n \rightarrow \infty . \\
& \text { (iv) } \mathbb{E}\left[\int_{0}^{T} e^{-\tilde{\Lambda} t}\left(u^{n}(t), v(t)\right)_{\mathbb{L}^{2}} d t\right] \rightarrow \mathbb{E}\left[\int_{0}^{T} e^{-\tilde{\Lambda} t}(u(t), v(t))_{\mathbb{L}^{2}} d t\right] \\
& \text { as } n \rightarrow \infty \text {. } \\
& (v) \mathbb{E}\left[\int_{0}^{T} e^{-\tilde{\Lambda} t}\left\|\sigma^{n}(t, v(t))\right\|_{L_{Q}\left(\mathbb{L}^{2}, \mathbb{L}^{2}\right)} d t\right] \rightarrow \mathbb{E}\left[\int_{0}^{T} e^{-\tilde{\Lambda} t}\|\sigma(t, v(t))\|_{L_{Q}\left(\mathbb{L}^{2}, \mathbb{L}^{2}\right)}^{2} d t\right] \\
& \text { as } n \rightarrow \infty \text {. } \\
& \text { (vi) } \mathbb{E}\left[\int_{0}^{T} e^{-\tilde{\Lambda} t} \int_{Z}\left\|H^{n}(v(t), z)\right\|_{\mathbb{L}^{2}}^{2} \lambda(d z) d t\right] \\
& \rightarrow \mathbb{E}\left[\int_{0}^{T} e^{-\tilde{\Lambda} t} \int_{Z}\|H(v(t), z)\|_{\mathbb{L}^{2}}^{2} \lambda(d z) d t\right] \quad \text { as } \quad n \rightarrow \infty .
\end{aligned}
$$

\section{Proof of Claim 2.}

(i) Note, as $v \in L^{2}\left(\Omega ; L^{\infty}\left(0, T ; \mathbb{L}^{2}(\mathcal{O})\right) \cap L^{2}\left(0, T ; \mathbb{H}_{0}^{1}(\mathcal{O})\right)\right), F(v(t)) \in L^{2}\left(\Omega ; L^{2}\left(0, T ; \mathbb{H}^{-1}(\mathcal{O})\right)\right)$. Since $u^{n} \rightarrow u$ weakly in $L^{2}\left(\Omega ; L^{2}\left(0, T ; \mathbb{H}_{0}^{1}(\mathcal{O})\right)\right)$, we infer that

$$
\left|\mathbb{E}\left[\int_{0}^{T} e^{-\tilde{\Lambda} t}\left(\left\langle F(v(t)), h u^{n}(t)\right\rangle-\langle F(v(t)), h u(t)\rangle\right) d t\right]\right|
$$




$$
\leq \mathbb{E}\left[\int_{0}^{T} e^{-\tilde{\Lambda} t}\left|\left\langle F(v(t)), h u^{n}(t)-h u(t)\right\rangle\right| d t\right] \rightarrow 0 \quad \text { as } \quad n \rightarrow \infty .
$$

This proves (i).

(ii) To prove (4.39), we first note

$$
\begin{aligned}
& \mid \mathbb{E}\left[\int_{0}^{T} e^{-\tilde{\Lambda} t}\left(\sigma^{n}\left(t, u^{n}(t)\right), \sigma^{n}(t, v(t))\right)_{L_{Q}\left(\mathbb{L}^{2}, \mathbb{L}^{2}\right)} d t\right] \\
& -\mathbb{E}\left[\int_{0}^{T} e^{-\tilde{\Lambda} t}\left(\sigma_{0}(t), \sigma(t, v(t))\right)_{L_{Q}\left(\mathbb{L}^{2}, \mathbb{L}^{2}\right)} d t\right] \mid \\
& =\mid \mathbb{E}\left[\int_{0}^{T} e^{-\tilde{\Lambda} t}\left(\sigma^{n}\left(t, u^{n}(t)\right)-\sigma_{0}(t), \sigma(t, v(t))\right)_{L_{Q}\left(\mathbb{L}^{2}, \mathbb{L}^{2}\right)} d t\right] \\
& +\mathbb{E}\left[\int_{0}^{T} e^{-\tilde{\Lambda} t}\left(\sigma^{n}\left(t, u^{n}(t)\right), \sigma^{n}(t, v(t))-\sigma(t, v(t))\right)_{L_{Q}\left(\mathbb{L}^{2}, \mathbb{L}^{2}\right)} d t\right] \mid \\
& \leq\left|\mathbb{E}\left[\int_{0}^{T} e^{-\tilde{\Lambda} t}\left(\sigma^{n}\left(t, u^{n}(t)\right)-\sigma_{0}(t), \sigma(t, v(t))\right)_{L_{Q}\left(\mathbb{L}^{2}, \mathbb{L}^{2}\right)} d t\right]\right| \\
& +\mathbb{E}\left[\int_{0}^{T} e^{-\tilde{\Lambda} t} \|\left(\sigma^{n}\left(t, u^{n}(t)\right)\left\|_{L_{Q}\left(\mathbb{L}^{2}, \mathbb{L}^{2}\right)}\right\| \sigma^{n}(t, v(t))-\sigma(t, v(t)) \|_{L_{Q}\left(\mathbb{L}^{2}, \mathbb{L}^{2}\right)} d t\right]\right. \\
& \leq\left|\mathbb{E}\left[\int_{0}^{T} e^{-\tilde{\Lambda} t}\left(\sigma^{n}\left(t, u^{n}(t)\right)-\sigma_{0}(t), \sigma(t, v(t))\right)_{L_{Q}\left(\mathbb{L}^{2}, \mathbb{L}^{2}\right)} d t\right]\right| \\
& +\left(\mathbb{E}\left[\int_{0}^{T} e^{-2 \tilde{\Lambda} t} \|\left(\sigma^{n}\left(t, u^{n}(t)\right) \|_{L_{Q}\left(\mathbb{L}^{2}, \mathbb{L}^{2}\right)}^{2} d t\right]\right)^{\frac{1}{2}}\right. \\
& \times\left(\mathbb{E}\left[\int_{0}^{T}\left\|\sigma^{n}(t, v(t))-\sigma(t, v(t))\right\|_{L_{Q}\left(\mathbb{L}^{2}, \mathbb{L}^{2}\right)}^{2} d t\right]\right)^{\frac{1}{2}} \\
& \leq\left|\mathbb{E}\left[\int_{0}^{T} e^{-\tilde{\Lambda} t}\left(\sigma^{n}\left(t, u^{n}(t)\right)-\sigma_{0}(t), \sigma(t, v(t))\right)_{L_{Q}\left(\mathbb{L}^{2}, \mathbb{L}^{2}\right)} d t\right]\right| \\
& +c\left(\mathbb{E}\left[\int_{0}^{T}\left\|\sigma^{n}(t, v(t))-\sigma(t, v(t))\right\|_{L_{Q}\left(\mathbb{L}^{2}, \mathbb{L}^{2}\right)}^{2} d t\right]\right)^{\frac{1}{2}},
\end{aligned}
$$

where by using Assumption 3.12 , and Proposition 4.3 we observe that

$$
\begin{aligned}
c: & =\sup _{n \in \mathbb{N}}\left(\mathbb{E}\left[\int_{0}^{T} e^{-2 \tilde{\Lambda} t} \|\left(\sigma^{n}\left(t, u^{n}(t)\right) \|_{L_{Q}\left(\mathbb{L}^{2}, \mathbb{L}^{2}\right)}^{2} d t\right]\right)\right. \\
& \leq \sup _{n \in \mathbb{N}}\left(\mathbb{E}\left[\int_{0}^{T} e^{-2 \tilde{\Lambda} t} \|\left(\sigma\left(t, u^{n}(t)\right) \|_{L_{Q}\left(\mathbb{L}^{2}, \mathbb{L}^{2}\right)}^{2} d t\right]\right)\right. \\
& \left.\leq K \sup _{n \in \mathbb{N}}\left(\mathbb{E}\left[\int_{0}^{T} e^{-2 \tilde{\Lambda} t}\left(1+\| u^{n}(t)\right) \|_{\mathbb{L}^{2}}^{2}\right) d t\right]\right) \\
& \left.\leq C_{T}+C_{T} \sup _{n \in \mathbb{N}} \mathbb{E}\left(\sup _{t \in[0, T]} \| u^{n}(t)\right) \|_{\mathbb{L}^{2}}^{2}\right)<\infty .
\end{aligned}
$$

Thus using (4.31), the first term on the right hand side of (4.44) tends to zero as $n \rightarrow$ $\infty$. Since $P_{n} \sigma(\cdot, v(\cdot))=\sigma^{n}(\cdot, v(\cdot))$, we now prove $\sigma^{n}(\cdot, v(\cdot)) \rightarrow \sigma(\cdot, v(\cdot))$ strongly in $L^{2}\left(\Omega ; L^{2}\left(0, T ; L_{Q}\left(\mathbb{L}^{2}, \mathbb{L}^{2}\right)\right)\right)$.

Let us fix $t \in[0, T]$ and $\omega \in \Omega$ and define the double sequence $a_{n, k}:=\|\left(\sigma^{n}(t, v(t))-\right.$ $\sigma(t, v(t))) Q^{\frac{1}{2}} \psi_{k} \|_{\mathbb{L}^{2}}^{2}$, where $\left\{\psi_{k}\right\}_{k \geq 1}$ is an orthonormal basis in $\mathbb{L}^{2}$. Define $b_{k}:=4\left\|\sigma(t, v(t)) Q^{\frac{1}{2}} \psi_{k}\right\|_{\mathbb{L}^{2}}^{2}$. Note, by Minkowski inequality and (4.18), $a_{n, k} \leq b_{k}$ for every $n$ and $k$. Moreover, $\sum_{k=1}^{\infty} b_{k}=4 \sum_{k=1}^{\infty}\left\|\sigma(t, v(t)) Q^{\frac{1}{2}} \psi_{k}\right\|_{\mathbb{L}^{2}}^{2}=4\|\sigma(t, v(t))\|_{L_{Q}\left(\mathbb{L}^{2}, \mathbb{L}^{2}\right)}^{2}<\infty$. Now using Lemma 4.1 for every $k$, we achieve

$$
\lim _{n \rightarrow \infty} a_{n}(k)=\lim _{n \rightarrow \infty}\left\|\left(\sigma^{n}(t, v(t))-\sigma(t, v(t))\right) Q^{\frac{1}{2}} \psi_{k}\right\|_{\mathbb{L}^{2}}^{2}
$$




$$
=\lim _{n \rightarrow \infty}\left\|\sigma^{n}(t, v(t)) Q^{\frac{1}{2}} \psi_{k}-\sigma(t, v(t)) Q^{\frac{1}{2}} \psi_{k}\right\|_{\mathbb{L}^{2}}^{2}=0 .
$$

Hence by Lebesgue Dominated Convergence Theorem (for double sequence),

$$
\lim _{n \rightarrow \infty} \sum_{k=1}^{\infty} a_{n, k}=0
$$

Thus for almost all $t \in[0, T], P$-a.e. $\omega \in \Omega$ we obtain

$$
\lim _{n \rightarrow \infty}\left\|\sigma^{n}(t, v(t))-\sigma(t, v(t))\right\|_{L_{Q}\left(\mathbb{L}^{2}, \mathbb{L}^{2}\right)}^{2}=0 .
$$

Furthermore, $\left\|\sigma^{n}(t, v(t))-\sigma(t, v(t))\right\|_{L_{Q}\left(\mathbb{L}^{2}, \mathbb{L}^{2}\right)}^{2} \leq 4\|\sigma(t, v(t))\|_{L_{Q}\left(\mathbb{L}^{2}, \mathbb{L}^{2}\right)}^{2}$ for almost all $t \in$ $[0, T], P$-a.e. $\omega \in \Omega$. Thus applying Lebesgue Dominated Convergence Theorem again we achieve,

$$
\lim _{n \rightarrow \infty} \mathbb{E}\left[\int_{0}^{T}\left\|\sigma^{n}(t, v(t))-\sigma(t, v(t))\right\|_{L_{Q}\left(\mathbb{L}^{2}, \mathbb{L}^{2}\right)}^{2} d t\right]=0,
$$

proving that $\sigma^{n}(\cdot, v(\cdot)) \rightarrow \sigma(\cdot, v(\cdot))$ strongly in $L^{2}\left(\Omega ; L^{2}\left(0, T ; L_{Q}\left(\mathbb{L}^{2}, \mathbb{L}^{2}\right)\right)\right)$. Hence, second term on the right hand side of (4.44) goes to zero as $n \rightarrow \infty$. Thus we have (4.39).

(iii) Let us consider

$$
\begin{aligned}
& \mid \mathbb{E}\left[\int_{0}^{T} e^{-\tilde{\Lambda} t} \int_{Z}\left(H^{n}\left(u^{n}(t), z\right), H^{n}(v(t), z)\right)_{\mathbb{L}^{2}} \lambda(d z) d t\right] \\
&-\quad \mathbb{E}\left[\int_{0}^{T} e^{-\tilde{\Lambda} t} \int_{Z}\left(H_{0}(t, z), H(v(t), z)\right)_{\mathbb{L}^{2}} \lambda(d z) d t\right] \mid \\
&=\mid \mathbb{E}\left[\int_{0}^{T} e^{-\tilde{\Lambda} t} \int_{Z}\left(H^{n}\left(u^{n}(t), z\right)-H_{0}(t, z), H(v(t), z)\right)_{\mathbb{L}^{2}} \lambda(d z) d t\right] \\
&+ \mathbb{E}\left[\int_{0}^{T} e^{-\tilde{\Lambda} t} \int_{Z}\left(H^{n}\left(u^{n}(t), z\right), H^{n}(v(t), z)-H(v(t), z)\right)_{\mathbb{L}^{2}} \lambda(d z) d t\right] \mid \\
& \leq\left|\mathbb{E}\left[\int_{0}^{T} e^{-\tilde{\Lambda} t} \int_{Z}\left(H^{n}\left(u^{n}(t), z\right)-H_{0}(t, z), H(v(t), z)\right)_{\mathbb{L}^{2}} \lambda(d z) d t\right]\right| \\
&+\mathbb{E}\left[\int_{0}^{T} e^{-\tilde{\Lambda} t} \int_{Z} \|\left(H^{n}\left(u^{n}(t), z\right)\left\|_{\mathbb{L}^{2}}\right\| H^{n}(v(t), z)-H(v(t), z) \|_{\left.\mathbb{L}^{2} \lambda(d z) d t\right]}\right.\right. \\
& \leq\left|\mathbb{E}\left[\int_{0}^{T} e^{-\tilde{\Lambda} t} \int_{Z}\left(H^{n}\left(u^{n}(t), z\right)-H_{0}(t, z), H(t, v(t))\right)_{\mathbb{L}^{2}} \lambda(d z) d t\right]\right| \\
&+\left(\mathbb{E}\left[\int_{0}^{T} e^{-2 \tilde{\Lambda} t} \int_{Z} \|\left(H^{n}\left(t, u^{n}(t)\right) \|_{\mathbb{L}^{2}}^{2} \lambda(d z) d t\right]\right)^{\frac{1}{2}}\right. \\
& \times\left(\mathbb{E}\left[\int_{0}^{T} \int_{Z}\left\|H^{n}(v(t), z)-H(v(t), z)\right\|_{\mathbb{L}^{2}}^{2} \lambda(d z) d t\right]\right)^{\frac{1}{2}} \\
& \leq\left|\mathbb{E}\left[\int_{0}^{T} e^{-\tilde{\Lambda} t} \int_{Z}\left(H^{n}\left(u^{n}(t), z\right)-H_{0}(t, z), H(v(t), z)\right)_{\mathbb{L}^{2}} \lambda(d z) d t\right]\right| \\
&+c\left(\mathbb{E}\left[\int_{0}^{T} \int_{Z}\left\|H^{n}(v(t), z)-H(v(t), z)\right\|_{\mathbb{L}^{2}}^{2} \lambda(d z) d t\right]\right)^{\frac{1}{2}}
\end{aligned}
$$

where by using Assumption 3.12, Proposition 4.3 we observe that

$$
\begin{aligned}
c: & =\sup _{n \in \mathbb{N}}\left(\mathbb{E}\left[\int_{0}^{T} e^{-2 \tilde{\Lambda} t} \int_{Z} \|\left(H^{n}\left(u^{n}(t), z\right) \|_{\mathbb{L}^{2}}^{2} \lambda(d z) d t\right]\right)\right. \\
& \leq \sup _{n \in \mathbb{N}}\left(\mathbb{E}\left[\int_{0}^{T} e^{-2 \tilde{\Lambda} t} \int_{Z} \|\left(H\left(u^{n}(t), z\right) \|_{\mathbb{L}^{2}}^{2} \lambda(d z) d t\right]\right)\right. \\
& \left.\leq K \sup _{n \in \mathbb{N}}\left(\mathbb{E}\left[\int_{0}^{T} e^{-2 \tilde{\Lambda} t}\left(1+\| u^{n}(t)\right) \|_{\mathbb{L}^{2}}^{2}\right) d t\right]\right)
\end{aligned}
$$




$$
\left.\leq C_{T}+C_{T} \sup _{n \in \mathbb{N}} \mathbb{E}\left(\sup _{t \in[0, T]} \| u^{n}(t)\right) \|_{\mathbb{L}^{2}}^{2}\right)<\infty .
$$

Thus using (4.32) first term on the right hand side of (4.47) tends to zero. Recalling $P_{n} H(v(\cdot), \cdot)=H^{n}(v(\cdot), \cdot)$, using Lemma 4.1 and (4.19), we have for almost all $t \in[0, T]$ and $P$-a.e. $\omega \in \Omega$, all $z \in Z$,

$$
\begin{aligned}
& \lim _{n \rightarrow \infty}\left\|H^{n}(v(t), z)-H(v(t), z)\right\|_{\mathbb{L}^{2}}=0 \text { and } \\
& \left\|H^{n}(v(t), z)-H(v(t), z)\right\|_{\mathbb{L}^{2}} \leq 2\|H(v(t), z)\|_{\mathbb{L}^{2}} .
\end{aligned}
$$

Hence by applying Lebesgue Dominated Convergence Theorem, we have

$$
\lim _{n \rightarrow \infty} \mathbb{E}\left[\int_{0}^{T} \int_{Z}\left\|H^{n}(v(t), z)-H(v(t), z)\right\|_{\mathbb{L}^{2}}^{2} \lambda(d z) d t\right]=0,
$$

proving that $H^{n}(v(\cdot), \cdot) \rightarrow H(v(\cdot), \cdot)$ strongly in $\mathbb{H}_{\lambda}^{2}\left([0, T] \times Z ; \mathbb{L}^{2}\right)$, which also implies that the second term on the right hand side of (4.47) goes to zero, ensuring (4.40).

(iv) Using (4.27) we directly have (4.41).

(v) It directly follows from (4.46).

(vi) It directly follows from (4.49).

Step IV : Consequences of monotonicity argument Using the monotonicity property of $F(\cdot)($ see Remark 3.15 and (3.20) $)$ and Assumption 3.12 we have for $v \in L^{2}\left(\Omega ; L^{\infty}\left(0, T ; \mathbb{L}^{2}(\mathcal{O})\right) \cap\right.$ $\left.L^{2}\left(0, T ; \mathbb{H}_{0}^{1}(\mathcal{O})\right)\right)$

$$
\begin{aligned}
& 2 \mathbb{E}\left[\int_{0}^{T} e^{-\tilde{\Lambda} t}\left\langle F\left(u^{n}(t)\right)-F(v(t)), h u^{n}(t)-h v(t)\right\rangle d t\right] \\
& -\mathbb{E}\left[\int_{0}^{T} e^{-\tilde{\Lambda} t}\left\|\sigma^{n}\left(t, u^{n}(t)\right)-\sigma^{n}(t, v(t))\right\|_{L_{Q}\left(\mathbb{L}^{2}, \mathbb{L}^{2}\right)}^{2} d t\right] \\
& -\mathbb{E}\left[\int_{0}^{T} e^{-\tilde{\Lambda} t} \int_{Z}\left\|H^{n}\left(u^{n}(t), z\right)-H^{n}(v(t), z)\right\|_{\mathbb{L}^{2}}^{2} \lambda(d z) d t\right] \\
& +\mathbb{E}\left[\int_{0}^{T} \tilde{\Lambda} e^{-\tilde{\Lambda} t}\left\|u^{n}(t)-v(t)\right\|_{\mathbb{L}^{2}}^{2} d t\right] \geq 0 .
\end{aligned}
$$

Splitting each of the inner products and norms and then on rearranging we obtain,

$$
\begin{aligned}
& -2 \mathbb{E}\left[\int_{0}^{T} e^{-\tilde{\Lambda} t}\left\langle F\left(u^{n}(t)\right), h u^{n}(t)-h v(t)\right\rangle d t\right]+\mathbb{E}\left[\int_{0}^{T} e^{-\tilde{\Lambda} t}\left\|\sigma^{n}\left(t, u^{n}(t)\right)\right\|_{L_{Q}\left(\mathbb{L}^{2}, \mathbb{L}^{2}\right)}^{2} d t\right] \\
& +\mathbb{E}\left[\int_{0}^{T} e^{-\tilde{\Lambda} t} \int_{Z}\left\|H^{n}\left(u^{n}(t), z\right)\right\|_{\mathbb{L}^{2}}^{2} \lambda(d z) d t\right]-\mathbb{E}\left[\int_{0}^{T} \tilde{\Lambda} e^{-\tilde{\Lambda} t}\left\|u^{n}(t)\right\|_{\mathbb{L}^{2}}^{2} d t\right] \\
& \leq-2 \mathbb{E}\left[\int_{0}^{T} e^{-\tilde{\Lambda} t}\left\langle F(v(t)), h u^{n}(t)-h v(t)\right\rangle d t\right]-\mathbb{E}\left[\int_{0}^{T} e^{-\tilde{\Lambda} t}\left\|\sigma^{n}(t, v(t))\right\|_{L_{Q}\left(\mathbb{L}^{2}, \mathbb{L}^{2}\right)}^{2} d t\right] \\
& +2 \mathbb{E}\left[\int_{0}^{T} e^{-\tilde{\Lambda} t}\left(\sigma^{n}\left(u^{n}(t)\right), \sigma^{n}(v(t))\right)_{L_{Q}\left(\mathbb{L}^{2}, \mathbb{L}^{2}\right)} d t\right] \\
& -\mathbb{E}\left[\int_{0}^{T} e^{-\tilde{\Lambda} t} \int_{Z}\left\|H^{n}(v(t), z)\right\|_{\mathbb{L}^{2}}^{2} \lambda(d z) d t\right] \\
& +2 \mathbb{E}\left[\int_{0}^{T} e^{-\tilde{\Lambda} t} \int_{Z}\left(H^{n}\left(u^{n}(t), z\right), H^{n}(v(t), z)\right)_{\mathbb{L}^{2}} \lambda(d z) d t\right] \\
& +\mathbb{E}\left[\int_{0}^{T} \tilde{\Lambda} e^{-\tilde{\Lambda} t}\|v(t)\|_{\mathbb{L}^{2}}^{2} d t\right]-2 \mathbb{E}\left[\int_{0}^{T} \tilde{\Lambda} e^{-\tilde{\Lambda} t}\left(u^{n}(t), v(t)\right)_{\mathbb{L}^{2}} d t\right] .
\end{aligned}
$$

In view of (4.38), (4.39)-(4.43), and taking limit in $n$ and using (4.37) we conclude that

$$
\begin{aligned}
& -2 \mathbb{E}\left[\int_{0}^{T} e^{-\tilde{\Lambda} t}\left\langle F_{0}(t), h u(t)-h v(t)\right\rangle d t\right]+\mathbb{E}\left[\int_{0}^{T} e^{-\tilde{\Lambda} t}\left\|\sigma_{0}(t)\right\|_{L_{Q}\left(\mathbb{L}^{2}, \mathbb{L}^{2}\right)}^{2} d t\right] \\
& +\mathbb{E}\left[\int_{0}^{T} e^{-\tilde{\Lambda} t} \int_{Z}\left\|H_{0}(t, z)\right\|_{\mathbb{L}^{2}}^{2} \lambda(d z) d t\right]-\mathbb{E}\left[\int_{0}^{T} \tilde{\Lambda} e^{-\tilde{\Lambda} t}\|u(t)\|_{\mathbb{L}^{2}}^{2} d t\right]
\end{aligned}
$$




$$
\begin{aligned}
\leq & -2 \mathbb{E}\left[\int_{0}^{T} e^{-\tilde{\Lambda} t}\langle F(v(t)), h u(t)-h v(t)\rangle d t\right]-\mathbb{E}\left[\int_{0}^{T} e^{-\tilde{\Lambda} t}\|\sigma(t, v(t))\|_{L_{Q}\left(\mathbb{L}^{2}, \mathbb{L}^{2}\right)}^{2} d t\right] \\
& +2 \mathbb{E}\left[\int_{0}^{T} e^{-\tilde{\Lambda} t}\left(\sigma_{0}(t), \sigma(v(t))\right)_{L_{Q}\left(\mathbb{L}^{2}, \mathbb{L}^{2}\right)} d t\right]-\mathbb{E}\left[\int_{0}^{T} e^{-\tilde{\Lambda} t} \int_{Z}\|H(v(t), z)\|_{\mathbb{L}^{2}}^{2} \lambda(d z) d t\right] \\
& +2 \mathbb{E}\left[\int_{0}^{T} e^{-\tilde{\Lambda} t} \int_{Z}\left(H_{0}(t, z), H(v(t), z)\right)_{\mathbb{L}^{2}} \lambda(d z) d t\right] \\
& +\mathbb{E}\left[\int_{0}^{T} \tilde{\Lambda} e^{-\tilde{\Lambda} t}\|v(t)\|_{\mathbb{L}^{2}}^{2} d t\right]-2 \mathbb{E}\left[\int_{0}^{T} \tilde{\Lambda} e^{-\tilde{\Lambda} t}(u(t), v(t))_{\mathbb{L}^{2}} d t\right] .
\end{aligned}
$$

Rearranging the terms

$$
\begin{aligned}
& -2 \mathbb{E}\left[\int_{0}^{T} e^{-\tilde{\Lambda} t}\left\langle F_{0}(t)-F(v(t)), h u(t)-h v(t)\right\rangle d t\right] \\
& +\mathbb{E}\left[\int_{0}^{T} e^{-\tilde{\Lambda} t}\left\|\sigma_{0}(t)-\sigma(t, v(t))\right\|_{L_{Q}\left(\mathbb{L}^{2}, \mathbb{L}^{2}\right)}^{2} d t\right] \\
& +\mathbb{E}\left[\int_{0}^{T} e^{-\tilde{\Lambda} t} \int_{Z}\left\|H_{0}(t, z)-H(v(t), z)\right\|_{\mathbb{L}^{2}}^{2} \lambda(d z) d t\right] \\
& -\mathbb{E}\left[\int_{0}^{T} \tilde{\Lambda} e^{-\tilde{\Lambda} t}\|u(t)-v(t)\|_{\mathbb{L}^{2}}^{2} d t\right] \leq 0 .
\end{aligned}
$$

The above estimate holds for any $v \in L^{2}\left(\Omega ; L^{\infty}\left(0, T ; \mathbb{L}^{2}(\mathcal{O})\right) \cap L^{2}\left(0, T ; \mathbb{H}_{0}^{1}(\mathcal{O})\right)\right)$. Choosing $v(\cdot)=$ $u(\cdot)$, we can immediately observe $\sigma_{0}(\cdot)=\sigma(\cdot, u(\cdot))$ and $H_{0}(\cdot, \cdot)=H(u(\cdot), \cdot)$. Now we take $v=u+$ $\lambda w$ in (4.52), where $\lambda>0$ and $w$ is an adapted process in $L^{2}\left(\Omega ; L^{\infty}\left(0, T ; \mathbb{L}^{2}(\mathcal{O})\right) \cap L^{2}\left(0, T ; \mathbb{H}_{0}^{1}(\mathcal{O})\right)\right)$. Then we have

$$
\begin{aligned}
& \lambda \mathbb{E}\left[\int_{0}^{T} e^{-\tilde{\Lambda} t}\langle F(u(t)+\lambda w(t)), h w(t)\rangle d t\right]+\lambda^{2} \tilde{\Lambda} \mathbb{E}\left[\int_{0}^{T} e^{-\tilde{\Lambda} t}\|w(t)\|_{\mathbb{L}^{2}}^{2} d t\right] \\
& \geq \lambda \mathbb{E}\left[\int_{0}^{T} e^{-\tilde{\Lambda} t}\left\langle F_{0}(t), h w(t)\right\rangle d t\right] .
\end{aligned}
$$

Dividing by $\lambda$ on both sides of the inequality above and letting $\lambda$ to go to 0 , we have by the hemicontinuity of $F(\cdot)$

$$
\mathbb{E}\left[\int_{0}^{T} e^{-\tilde{\Lambda} t}\left\langle F(u(t))-F_{0}(t), h w(t)\right\rangle d t\right] \geq 0 .
$$

Since $w$ is arbitrary and $h$ is a positive, bounded, continuously differentiable function, $F_{0}(t)=$ $F(u(t))$. This proves the existence of a strong solution.

\section{Step V : Uniqueness:}

If the pairs $(u, \hat{z})$ and $(v, \tilde{z})$ are two solutions to (1.1)-(1.3), then $w(t):=u(t)-v(t)$ and $\zeta(t):=$ $\hat{z}(t)-\tilde{z}(t)$ solves the stochastic integral equation

$$
\begin{aligned}
d w(t)+A w(t) d t+g \nabla \zeta(t) d t & \\
= & {[B(v(t))-B(u(t))] d t+(\sigma(t, u(t))-\sigma(t, v(t))) d W(t) } \\
& +\int_{Z}(H(u(t-), z)-H(v(t-), z)) \tilde{N}(d t, d z) .
\end{aligned}
$$

and

$$
d \zeta(t)+\operatorname{Div}(h w(t)) d t=0
$$

Define the stopping time as:

$$
\tau_{N}=\inf \left\{t \geq 0:\|w(t)\|_{\mathbb{L}^{2}}^{2}+\|\zeta(t)\|_{L^{2}}^{2}+\int_{0}^{t}\|w(s)\|_{\mathbb{H}_{0}^{1}}^{2} d s>N\right\} .
$$

Applying Itô's lemma to the process $\|w(t)\|_{\mathbb{L}^{2}}^{2}$

$$
\left\|w\left(t \wedge \tau_{N}\right)\right\|_{\mathbb{L}^{2}}^{2}+2 \alpha \int_{0}^{t \wedge \tau_{N}}\|w(s)\|_{\mathbb{H}_{0}^{1}}^{2} d s+2 g \int_{0}^{t \wedge \tau_{N}}\langle\nabla \zeta(s), w(s)\rangle d s
$$




$$
\begin{aligned}
= & \|w(0)\|_{\mathbb{L}^{2}}^{2}+2 \int_{0}^{t \wedge \tau_{N}}\left[(B(v(s))-B(u(s)), u(s)-v(s))_{\mathbb{L}^{2}}\right] d s \\
& +2 \int_{0}^{t \wedge \tau_{N}}(\sigma(s, u(s))-\sigma(s, v(s)) d W(s), w(s))_{\mathbb{L}^{2}} \\
& +\int_{0}^{t \wedge \tau_{N}}\|\sigma(s, u(s))-\sigma(s, v(s))\|_{L_{Q}\left(\mathbb{L}^{2}, \mathbb{L}^{2}\right)}^{2} d s \\
& +\int_{0}^{t \wedge \tau_{N}} \int_{Z}\|H(u(s-), z)-H(v(s-), z)\|_{\mathbb{L}^{2}}^{2} N(d s, d z) \\
& +2 \int_{0}^{t \wedge \tau_{N}} \int_{Z}((H(u(s-), z)-H(v(s-), z)), w(s-))_{\mathbb{L}^{2}} \tilde{N}(d s, d z) .
\end{aligned}
$$

Using the result from Lemma 3.2, inequality (4.10) and $w(0)=0$,

$$
\begin{aligned}
& \left\|w\left(t \wedge \tau_{N}\right)\right\|_{\mathbb{L}^{2}}^{2}+2 \alpha \int_{0}^{t \wedge \tau_{N}}\|w(s)\|_{\mathbb{H}_{0}^{1}}^{2} d s \\
& \leq \frac{2 g^{2}}{\alpha} \int_{0}^{t \wedge \tau_{N}}\|\zeta(s)\|_{L^{2}}^{2} d s+\frac{\alpha}{2} \int_{0}^{t \wedge \tau_{N}}\|w(s)\|_{\mathbb{H}_{0}^{1}}^{2} d s \\
& \quad+2 \int_{0}^{t \wedge \tau_{N}}(\sigma(s, u(s))-\sigma(s, v(s)) d W(s), w(s))_{\mathbb{L}^{2}} \\
& \quad+\int_{0}^{t \wedge \tau_{N}}\|\sigma(s, u(s))-\sigma(s, v(s))\|_{L_{Q}\left(\mathbb{L}^{2}, \mathbb{L}^{2}\right)}^{2} d s \\
& \quad+\int_{0}^{t \wedge \tau_{N}} \int_{Z}\|H(u(s-), z)-H(v(s-), z)\|_{\mathbb{L}^{2}}^{2} N(d s, d z) \\
& \quad+2 \int_{0}^{t \wedge \tau_{N}} \int_{Z}((H(u(s-), z)-H(v(s-), z)), w(s-))_{\mathbb{L}^{2}} \tilde{N}(d s, d z) .
\end{aligned}
$$

Taking (4.54) inner product with $\zeta(t):=\hat{z}(t)-\tilde{z}(t)$ and using $\zeta(0)=0$, we have as in equation (4.15)

$$
\begin{aligned}
\left\|\zeta\left(t \wedge \tau_{N}\right)\right\|_{L^{2}}^{2} \leq & M \int_{0}^{t \wedge \tau_{N}}\|w(s)\|_{\mathbb{L}^{2}}^{2} d s+\left(\frac{2 \mu^{2}}{\alpha}+M\right) \int_{0}^{t \wedge \tau_{N}}\|\zeta(s)\|_{L^{2}}^{2} d s \\
& +\frac{\alpha}{2} \int_{0}^{t \wedge \tau_{N}}\|w(s)\|_{\mathbb{H}_{0}^{1}}^{2} d s .
\end{aligned}
$$

Let $C=\frac{2 g^{2}}{\alpha}+\frac{2 \mu^{2}}{\alpha}+M$. Adding equations (4.56) and (4.57), then taking expectation and using Itô-Lévy isometry we achieve

$$
\begin{aligned}
& \mathbb{E}\left[\left\|w\left(t \wedge \tau_{N}\right)\right\|_{\mathbb{L}^{2}}^{2}+\left\|\zeta\left(t \wedge \tau_{N}\right)\right\|_{L^{2}}^{2}\right]+\mathbb{E}\left[\int_{0}^{t \wedge \tau_{N}} \alpha\|w(s)\|_{\mathbb{H}_{0}^{1}}^{2} d s\right] \\
& \leq C \mathbb{E}\left[\int_{0}^{t \wedge \tau_{N}}\left(\|w(s)\|_{\mathbb{L}^{2}}^{2}+\|\zeta(s)\|_{L^{2}}^{2}\right) d s\right] \\
& \quad+\mathbb{E}\left[\int_{0}^{t \wedge \tau_{N}}\|\sigma(s, u(s))-\sigma(s, v(s))\|_{L_{Q}\left(\mathbb{L}^{2}, \mathbb{L}^{2}\right)}^{2} d s\right] \\
& \quad+\mathbb{E}\left[\int_{0}^{t \wedge \tau_{N}} \int_{Z}\|H(u(s), z)-H(v(s), z)\|_{\mathbb{L}^{2}}^{2} \lambda(d z) d s\right] .
\end{aligned}
$$

Applications of Assumption 3.12 and Gronwall's inequality yield

$$
\mathbb{E}\left[\left\|w\left(t \wedge \tau_{N}\right)\right\|_{\mathbb{L}^{2}}^{2}+\left\|\zeta\left(t \wedge \tau_{N}\right)\right\|_{L^{2}}^{2}\right]+\alpha \mathbb{E}\left[\int_{0}^{t \wedge \tau_{N}}\|w(s)\|_{\mathbb{H}_{0}^{1}}^{2} d s\right] \leq 0 .
$$

As $N \rightarrow \infty, t \wedge \tau_{N} \rightarrow t$ a.s., and hence the uniqueness of pathwise strong solution follows. 


\section{Stochastic Optimal Control}

5.1. Preliminaries. In this Subsection we provide some definitions and known results borrowed from Métivier [34] and Aldous [2].

Definition 5.1. Let $(\mathbb{S}, \rho)$ be a separable and complete metric space. Let $u \in D([0, T] ; \mathbb{S})$ and let $\delta>0$ be given. A modulus of $u$ is defined by

$$
w_{[0, T], \mathbb{S}}(u, \delta):=\inf _{\Pi_{\delta}} \max _{t_{i} \in \bar{\omega}} \sup _{t_{i} \leq s<t<t_{i+1} \leq T} \rho(u(t), u(s)),
$$

where $\Pi_{\delta}$ is the set of all increasing sequences $\bar{\omega}=\left\{0=t_{0}<t_{1}<\ldots<t_{n}=T\right\}$ with the following property

$$
t_{i+1}-t_{i} \geq \delta, \quad i=0, \ldots, n-1 .
$$

Theorem 5.2. A set $A \subset D([0, T] ; \mathbb{S})$ has compact closure iff it satisfies the following two conditions:

(a) there exists a dense subset $I \subset[0, T]$ such that for every $t \in I$ the set $\{u(t), u \in A\}$ has compact closure in $\mathbb{S}$,

(b) $\lim _{\delta \rightarrow 0} \sup _{u \in A} w_{[0, T], \mathbb{S}}(u, \delta)=0$.

Let us consider the ball $\mathbb{B}:=\left\{x \in \mathbb{L}^{2}(\mathcal{O}):\|x\|_{\mathbb{L}^{2}} \leq r\right\}$. If $\mathbb{B}_{w}$ denote the ball $\mathbb{B}$ endowed with the weak topology of $\mathbb{L}^{2}$, then it is well-known that $\mathbb{B}_{w}$ is metrizable. Let $D\left([0, T] ; \mathbb{B}_{w}\right)$ be the space of weakly càdlàg functions $u:[0, T] \rightarrow \mathbb{L}^{2}$ such that $\sup _{t \in[0, T]}\|u(t)\|_{\mathbb{L}^{2}} \leq r$. Note that, the space $D\left([0, T] ; \mathbb{B}_{w}\right)$ is metrizable as well. Then we have the following lemma from Lemma 2 of [37].

Lemma 5.3. Let $u_{n}:[0, T] \rightarrow \mathbb{L}^{2}, n \in \mathbb{N}$ be functions such that

(1) $\sup _{n \in \mathbb{N}} \sup _{s \in[0, T]}\left\|u_{n}(s)\right\|_{\mathbb{L}^{2}} \leq r$,

(2) $u_{n} \rightarrow u$ in $D\left([0, T] ; \mathbb{H}^{-1}\right)$.

Then $u, u_{n} \in D\left([0, T] ; \mathbb{B}_{w}\right)$ and $u_{n} \rightarrow u$ in $D\left([0, T] ; \mathbb{B}_{w}\right)$ as $n \rightarrow \infty$.

We now deal with the following functional spaces endowed with the respective topologies:

$$
D\left([0, T] ; \mathbb{H}^{-1}(\mathcal{O})\right)_{J}:=\text { the the space of càdlàg functions } u:[0, T] \rightarrow \mathbb{H}^{-1}(\mathcal{O})
$$

with the extended Skorokhod topology $\tau_{1}$,

$$
L_{w}^{2}\left(0, T ; \mathbb{H}_{0}^{1}(\mathcal{O})\right):=\text { the space } L^{2}\left(0, T ; \mathbb{H}_{0}^{1}(\mathcal{O})\right) \text { with the weak topology } \tau_{2},
$$

$D\left([0, T] ; \mathbb{L}_{w}^{2}(\mathcal{O})\right):=$ the the space of all weakly càdlàg functions $u:[0, T] \rightarrow$

$\mathbb{L}^{2}(\mathcal{O})$ with the weakest topology $\tau_{3}$ such that for all $h \in \mathbb{L}^{2}(\mathcal{O})$ the mappings

$$
D\left([0, T] ; \mathbb{L}_{w}^{2}(\mathcal{O})\right) \ni u \mapsto(u(\cdot), h)_{\mathbb{L}^{2}} \in D([0, T] ; \mathbb{R})
$$

are continuous.

$$
L^{2}\left(0, T ; \mathbb{L}^{2}(\mathcal{O})\right) \text { is endowed with its strong topology } \tau_{4} .
$$

We take the path space $\mathcal{Z}=D\left([0, T] ; \mathbb{H}^{-1}(\mathcal{O})\right)_{J} \cap D\left([0, T] ; \mathbb{L}_{w}^{2}(\mathcal{O})\right) \cap L_{w}^{2}\left(0, T ; \mathbb{H}_{0}^{1}(\mathcal{O})\right) \cap L^{2}\left(0, T ; \mathbb{L}^{2}(\mathcal{O})\right)$ and $\tau$ be the supremum of the corresponding topologies.

Theorem 5.4. A set $\mathcal{K} \subset \mathcal{Z}$ is $\tau$-relatively compact if the following three conditions hold:

(a) $\forall u \in \mathcal{K}$ and all $t \in[0, T], u(t) \in \mathbb{L}^{2}(\mathcal{O})$ and $\sup _{u \in \mathcal{K}} \sup _{s \in[0, T]}\|u(s)\|_{\mathbb{L}^{2}}<\infty$,

(b) $\sup _{u \in \mathcal{K}} \int_{0}^{T}\|u(s)\|_{\mathbb{H}_{0}^{1}}^{2} d s<\infty$, i.e. $\mathcal{K}$ is bounded in $L^{2}\left(0, T ; \mathbb{H}_{0}^{1}(\mathcal{O})\right)$,

(c) $\lim _{\delta \rightarrow 0} \sup _{u \in \mathcal{K}} w_{[0, T], \mathbb{H}^{-1}(\mathcal{O})}(u, \delta)=0$.

For proof see Lemma 3.3 in [11], Lemma 4.1 in [36], Theorem 2 of [37], Lemma 2.7 in [35]

Definition 5.5. Let $(\mathbb{S}, \rho)$ be a separable and complete metric space. Let $(\Omega, \mathcal{F}, P)$ be a probability space with the filtration $F:=\left(\mathcal{F}_{t}\right)_{t \in[0, T]}$ satisfying the usual hypotheses, and let $\left(X_{n}\right)_{n \in \mathbb{N}}$ be a sequence of càdlàg, $F$-adapted and $\mathbb{S}$-valued processes. $\left(X_{n}\right)_{n \in \mathbb{N}}$ is said to satisfy the Aldous 
condition iff $\forall \epsilon>0, \forall \eta>0, \exists \delta>0$ such that for every sequence $\left(\tau_{n}\right)_{n \in \mathbb{N}}$ of stopping times with $\tau_{n} \leq T$

$$
\sup _{n \in \mathbb{N}} \sup _{0 \leq \theta \leq \delta} P\left\{\rho\left(X_{n}\left(\tau_{n}+\theta\right), X_{n}\left(\tau_{n}\right)\right) \geq \eta\right\} \leq \epsilon .
$$

Lemma 5.6. Let $\left(X_{n}\right)_{n \in \mathbb{N}}$ satisfies the Aldous condition. Let $\mathbb{P}^{n}$ be the law of $X_{n}$ on $D([0, T] ; \mathbb{S}), n \in$ $\mathbb{N}$. Then for every $\epsilon>0$ there exists a subset $A_{\epsilon} \subset D([0, T] ; \mathbb{S})$ such that

$$
\sup _{n \in \mathbb{N}} \mathbb{P}^{n}\left(A_{\epsilon}\right) \geq 1-\epsilon \quad \text { and } \quad \lim _{\delta \rightarrow 0} \sup _{u \in A_{\epsilon}} w_{[0, T], \mathbb{S}}(u, \delta)=0 .
$$

We will use the following lemma given in [34] and [37.

Lemma 5.7. Let $\left(E,\|\cdot\|_{E}\right)$ be a separable Banach space and let $\left(X_{n}\right)_{n \in \mathbb{N}}$ be a sequence of E-valued random variables. Assume that for every sequence $\left(\tau_{n}\right)_{n \in \mathbb{N}}$ of $F$-stopping times with $\tau_{n} \leq T$ and for every $n \in \mathbb{N}$ and $\theta \geq 0$ the following condition holds

$$
\mathbb{E}\left[\left\|X_{n}\left(\tau_{n}+\theta\right)-X_{n}\left(\tau_{n}\right)\right\|_{E}^{\alpha}\right] \leq C \theta^{\beta},
$$

for some $\alpha, \beta>0$ and some constant $C>0$. Then the sequence $\left(X_{n}\right)_{n \in \mathbb{N}}$ satisfies the Aldous condition in the space $E$.

We use the tightness condition for the Prokhorov-Varadarajan theorem which states that a sequence of measures $\left(\tilde{\mathbb{P}}^{n}\right)_{n \in \mathbb{N}}$ is tight on a topological space $E$ if for every $\epsilon>0$ there exists a compact set $K_{\epsilon} \subset E$ such that $\sup _{n} \tilde{\mathbb{P}}^{n}\left(E \backslash K_{\epsilon}\right) \leq \epsilon$. Hence the tightness of measure in $\mathcal{Z}$ is given by the following theorem.

Theorem 5.8. Let $\left(X_{n}\right)_{n \in \mathbb{N}}$ be a sequence of càdlàg $F$-adapted $\mathbb{H}^{-1}(\mathcal{O})$-valued processes such that

(a) there exists a positive constant $C_{1}$ such that

$$
\sup _{n \in \mathbb{N}} \mathbb{E}\left[\sup _{s \in[0, T]}\left\|X_{n}(s)\right\|_{\mathbb{L}^{2}}\right] \leq C_{1},
$$

(b) there exists a positive constant $C_{2}$ such that

$$
\sup _{n \in \mathbb{N}} \mathbb{E}\left[\int_{0}^{T}\left\|X_{n}(s)\right\|_{\mathbb{H}_{0}^{1}}^{2} d s\right] \leq C_{2},
$$

(c) $\left(X_{n}\right)_{n \in \mathbb{N}}$ satisfies the Aldous condition in $\mathbb{H}^{-1}(\mathcal{O})$.

Let $\tilde{\mathbb{P}}^{n}$ be the law of $X_{n}$ on $\mathcal{Z}$. Then for every $\epsilon>0$ there exists a compact subset $K_{\epsilon}$ of $\mathcal{Z}$ such that

$$
\tilde{\mathbb{P}}^{n}\left(K_{\epsilon}\right) \geq 1-\epsilon,
$$

and the sequence of measures $\left\{\tilde{\mathbb{P}}^{n}, n \in \mathbb{N}\right\}$ is said to be tight on $(\mathcal{Z}, \tau)$.

For proof see Corollary 1, 37.

5.2. Martingale Problem. We now consider the stochastic tidal dynamics equation with Lévy forcing as defined in Section 3 with initial value control as

$$
\begin{aligned}
& d u(t)+[A u(t)+B(u(t))+g \nabla \hat{z}(t)] d t=f(t) d t+\sigma(t, u(t)) d W(t) \\
& \quad+\int_{Z} H(u(t-), z) \tilde{N}(d t, d z), \\
& d \hat{z}(t)+D i v(h u(t)) d t=0, \\
& u(0)=u_{0}+U, \quad \hat{z}(0)=\hat{z}_{0},
\end{aligned}
$$

where $f \in L^{2}\left(\Omega ; L^{2}\left(0, T ; \mathbb{L}^{2}(\mathcal{O})\right)\right), u_{0}, U \in L^{2}\left(\Omega ; \mathbb{L}^{2}(\mathcal{O})\right)$ and $\hat{z}_{0} \in L^{2}\left(\Omega ; L^{2}(\mathcal{O})\right)$. We assume that $\sigma$ and $H$ satisfy Assumption 3.12

Definition 5.9. A martingale solution of (5.4)-(5.6) is a system $(\bar{\Omega}, \overline{\mathcal{F}}, \bar{F}, \bar{P}, \bar{u}, \bar{z}, \bar{U}, \bar{N}, \bar{W})$, where

- $(\bar{\Omega}, \overline{\mathcal{F}}, \bar{F}, \bar{P})$ is a filtered probability space with a filtration $\bar{F}=\left\{\overline{\mathcal{F}}_{t}\right\}_{t \geq 0}$,

- $\bar{N}$ is a time homogeneous Poisson random measure over $(\bar{\Omega}, \overline{\mathcal{F}}, \bar{F}, \bar{P})$ with the intensity measure $\lambda$, 
- $\bar{W}$ is a cylindrical Wiener process over $(\bar{\Omega}, \overline{\mathcal{F}}, \bar{F}, \bar{P})$,

- $\bar{U}$ is measurable with $\bar{P}$ - a.e. $\omega \in \bar{\Omega}, \bar{U}(\omega) \in \mathbb{L}^{2}(\mathcal{O})$,

- $\bar{u}, \bar{z}$ are progressively measurable processes with $\bar{P}$ - a.e. $\omega \in \bar{\Omega}$, the paths

$$
\begin{aligned}
& \bar{u}(\cdot, \omega) \in D\left([0, T] ; \mathbb{H}^{-1}(\mathcal{O})\right) \cap D\left([0, T] ; \mathbb{L}_{w}^{2}(\mathcal{O})\right) \cap L_{w}^{2}\left(0, T ; \mathbb{H}_{0}^{1}(\mathcal{O})\right) \cap L^{2}\left(0, T ; \mathbb{L}^{2}(\mathcal{O})\right), \\
& \bar{z}(\cdot, \omega) \in L^{2}\left(0, T ; L^{2}(\mathcal{O})\right) \cap C\left([0, T] ; H^{-1}(\mathcal{O})\right),
\end{aligned}
$$

such that for all $t \in[0, T]$, for all $v \in \mathbb{H}_{0}^{1}(\mathcal{O})$ and for all $w \in L^{2}(\mathcal{O})$, the following identities hold $\bar{P}$-a.s.

$$
\begin{aligned}
& (\bar{u}(t), v)_{\mathbb{L}^{2}}+\int_{0}^{t}(A \bar{u}(s), v)_{\mathbb{L}^{2}} d s+\int_{0}^{t}(B(\bar{u}(s)), v)_{\mathbb{L}^{2}} d s+\int_{0}^{t}\langle g \nabla \bar{z}(s), v\rangle d s \\
& =\left(u_{0}, v\right)_{\mathbb{L}^{2}}+(\bar{U}, v)_{\mathbb{L}^{2}}+\int_{0}^{t}(f(s), v)_{\mathbb{L}^{2}} d s \\
& \quad+\int_{0}^{t}(\sigma(s, \bar{u}(s)) d \bar{W}(s), v)_{\mathbb{L}^{2}}+\int_{0}^{t} \int_{Z}(H(\bar{u}(s-), z), v)_{\mathbb{L}^{2}} \tilde{\bar{N}}(d s, d z), \\
& (\bar{z}(t), w)_{L^{2}}+\int_{0}^{t}(\operatorname{Div}(h \bar{u}(s)), w)_{L^{2}} d s=\left(z_{0}, w\right)_{L^{2}},
\end{aligned}
$$

where $\langle\cdot, \cdot\rangle$ denotes the duality pairing between $\mathbb{H}_{0}^{1}(\mathcal{O})$ and $\mathbb{H}^{-1}(\mathcal{O})$.

Equivalent infinite dimensional martingale formulations are available in the literature [e.g. see Theorem 9 of Sritharan [45]. Similar formulations, in the finite dimensional case, are known due to Stroock and Varadhan [46] and Viot 50. Equivalence between infinite dimensional version of Stroock-Varadhan martingale formulations and weak formulations as in the spirit of Definition 5.9 can be found in Theorem 10 of Sritharan [45.

5.3. Existence of Martingale Solution. We will prove the existence of a martingale solution using the Galerkin approximations as explained in Section 4. We write

$$
\begin{aligned}
u^{n}(t)= & u_{0}^{n}+U^{n}-\int_{0}^{t}\left(A u^{n}(s)+B\left(u^{n}(s)\right)+g \nabla \hat{z}^{n}(s)-f(s)\right) d s \\
& +\int_{0}^{t} \sigma^{n}\left(s, u^{n}(s)\right) d W^{n}(s)+\int_{0}^{t} \int_{Z} H^{n}\left(u^{n}(s-), z\right) \tilde{N}(d s, d z) . \\
\hat{z}^{n}(t)= & \hat{z}_{0}^{n}-\int_{0}^{t} \operatorname{Div}\left(h u^{n}(s)\right) d s .
\end{aligned}
$$

For each $n \in \mathbb{N}$, we use the measures $\mathcal{L}\left(u^{n}\right)$ and $\mathcal{L}\left(\hat{z}^{n}\right)$ defined on $(\mathcal{Z}, \tau)$ and $L^{2}\left(0, T ; L^{2}(\mathcal{O})\right)$ respectively by the solution $\left(u^{n}, \hat{z}^{n}\right)$ of the Galerkin equations (5.9)-(5.10).

Lemma 5.10. The set of measures $\left\{\mathcal{L}\left(u^{n}\right), n \in \mathbb{N}\right\}$ is tight on $(\mathcal{Z}, \tau)$.

Proof. We will prove the tightness of the measures using the tightness criterion given in Theorem 5.8. From Proposition 4.3, conditions (a) and (b) of Theorem 5.8 are satisfied. Now we need to verify that condition (c) of Theorem 5.8 is also satisfied. Using Lemma 5.7 we will show that sequence $\left(u^{n}\right)_{n \in \mathbb{N}}$ satisfy Aldous condition in the space $\mathbb{H}^{-1}(\mathcal{O})$. Let $\theta>0$. Let $\left(\tau_{n}\right)_{n \in \mathbb{N}}$ be a sequence of stopping times where $0 \leq \tau_{n} \leq \tau_{n}+\theta \leq T$. Let us recall (5.9) as:

$$
\begin{aligned}
& u^{n}(t)=u_{0}^{n}+U^{n}-\int_{0}^{t} A u^{n}(s) d s-\int_{0}^{t} B\left(u^{n}(s)\right) d s-\int_{0}^{t} g \nabla \hat{z}^{n}(s) d s \\
& +\int_{0}^{t} f(s) d s+\int_{0}^{t} \sigma^{n}\left(s, u^{n}(s)\right) d W^{n}(s)+\int_{0}^{t} \int_{Z} H^{n}\left(u^{n}(s-), z\right) \tilde{N}(d s, d z) \\
& =J_{1}^{n}+J_{2}^{n}+J_{3}^{n}(t)+J_{4}^{n}(t)+J_{5}^{n}(t)+J_{6}^{n}(t)+J_{7}^{n}(t)+J_{8}^{n}(t), \quad t \in[0, T] .
\end{aligned}
$$

Now we will show that (5.2) holds for each $J_{i}^{n}, i \in\{1,2, \ldots, 8\}$.

First note, since the terms $J_{1}^{n}$ and $J_{2}^{n}$ are independent of time, clearly (5.2) is satisfied for any $\alpha, \beta>0$.

Now consider the term $J_{3}^{n}(t)$. Since $A: \mathbb{H}_{0}^{1}(\mathcal{O}) \rightarrow \mathbb{H}^{-1}(\mathcal{O})$, therefore for all $v \in \mathbb{H}_{0}^{1}(\mathcal{O})$

$$
\langle A u, v\rangle=(A u, v)_{\mathbb{L}^{2}} \leq C_{1}\|u\|_{\mathbb{H}_{0}^{1}}\|v\|_{\mathbb{H}_{0}^{1}} \text {. }
$$


Hence, using the above inequality

$$
\|A u\|_{\mathbb{H}^{-1}} \leq C_{1}\|u\|_{\mathbb{H}_{0}^{1}}
$$

Therefore by using (5.11), Hölder's inequality and (4.6), $J_{3}^{n}$ can be estimated as

$$
\begin{aligned}
& \mathbb{E}\left[\left\|J_{3}^{n}\left(\tau_{n}+\theta\right)-J_{3}^{n}\left(\tau_{n}\right)\right\|_{\mathbb{H}^{-1}}\right]=\mathbb{E}\left[\left\|\int_{\tau_{n}}^{\tau_{n}+\theta} A u^{n}(s) d s\right\|_{\mathbb{H}^{-1}}\right] \\
& \leq C_{1} \mathbb{E}\left[\int_{\tau_{n}}^{\tau_{n}+\theta}\left\|u^{n}(s)\right\|_{\mathbb{H}_{0}^{1}} d s\right] \leq C_{1} \mathbb{E}\left[\theta^{1 / 2}\left(\int_{0}^{T}\left\|u^{n}(s)\right\|_{\mathbb{H}_{0}^{1}}^{2} d s\right)^{1 / 2}\right] \\
& \leq C_{1} \theta^{1 / 2}\left[\mathbb{E}\left(\int_{0}^{T}\left\|u^{n}(s)\right\|_{\mathbb{H}_{0}^{1}}^{2} d s\right)\right]^{1 / 2} \leq c_{2} \theta^{1 / 2} .
\end{aligned}
$$

Thus $J_{3}^{n}$ satisfies (5.2) with $\alpha=1$ and $\beta=\frac{1}{2}$.

We next consider the term $J_{4}^{n}(t)$. In Proposition 4.4 for $p=4$ we note that $w^{0} \in L^{8}\left(\Omega ; L^{8}\right.$ $\left.\left(0, T ; \mathbb{L}^{8}(\mathcal{O})\right)\right) \subset L^{4}\left(\Omega ; L^{4}\left(0, T ; \mathbb{L}^{4}(\mathcal{O})\right)\right)$. Again by Proposition 4.4, for $p=4$, we obtain $\mathbb{E}\left[\int_{0}^{T}\left\|u^{n}(s)\right\|_{\mathbb{L}^{2}}^{2}\left\|u^{n}(s)\right\|_{\mathbb{H}_{0}^{1}}^{2} d s\right] \leq C_{1(4)}$. By the estimate (3.5) we have $\mathbb{E}\left[\int_{0}^{T}\left\|u^{n}(s)\right\|_{\mathbb{L}^{4}}^{4} d s\right] \leq C_{1(4)}$. Using the embedding $\mathbb{H}_{0}^{1}(\mathcal{O}) \hookrightarrow \mathbb{L}^{4}(\mathcal{O}) \hookrightarrow \mathbb{L}^{2}(\mathcal{O}) \hookrightarrow \mathbb{H}^{-1}(\mathcal{O})$, property of the operator B in (3.11), Minskowskii and Hölder's inequalities and Proposition 4.4 (for $p=4$ ) we estimate $J_{4}^{n}$ as

$$
\begin{aligned}
& \mathbb{E}\left[\left\|J_{4}^{n}\left(\tau_{n}+\theta\right)-J_{4}^{n}\left(\tau_{n}\right)\right\|_{\mathbb{H}^{-1}}\right]=\mathbb{E}\left[\left\|\int_{\tau_{n}}^{\tau_{n}+\theta} B\left(u^{n}(s)\right) d s\right\|_{\mathbb{H}^{-1}}\right] \\
& \quad \leq \mathbb{E}\left[\int_{\tau_{n}}^{\tau_{n}+\theta}\left\|B\left(u^{n}(s)\right)\right\|_{\mathbb{H}^{-1}} d s\right] \leq c \mathbb{E}\left[\int_{\tau_{n}}^{\tau_{n}+\theta}\left\|B\left(u^{n}(s)\right)\right\|_{\mathbb{L}^{2}} d s\right] \\
& \leq c C_{2} \mathbb{E}\left[\int_{\tau_{n}}^{\tau_{n}+\theta}\left\|u^{n}(s)+w^{0}(s)\right\|_{\mathbb{L}^{4}}^{2} d s\right] \\
& \quad \leq 2 c C_{2} \mathbb{E}\left[\int_{\tau_{n}}^{\tau_{n}+\theta}\left(\left\|u^{n}(s)\right\|_{\mathbb{L}^{4}}^{2}+\left\|w^{0}(s)\right\|_{\mathbb{L}^{4}}^{2}\right) d s\right] \\
& \quad \leq c_{3} \theta^{1 / 2}\left(\mathbb{E}\left[\int_{\tau_{n}}^{\tau_{n}+\theta}\left\|u^{n}(s)\right\|_{\mathbb{L}^{4}}^{4} d s\right]\right)^{1 / 2}+c_{3} \theta^{1 / 2}\left(\mathbb{E}\left[\int_{\tau_{n}}^{\tau_{n}+\theta}\left\|w^{0}(s)\right\|_{\mathbb{L}^{4}}^{4} d s\right]\right)^{1 / 2} \\
& \quad \leq c_{3} \theta^{1 / 2}\left(\mathbb{E}\left[\int_{0}^{T}\left\|u^{n}(s)\right\|_{\mathbb{L}^{4}}^{4} d s\right]\right)^{1 / 2}+c_{3} \theta^{1 / 2}\left(\mathbb{E}\left[\int_{0}^{T}\left\|w^{0}(s)\right\|_{\mathbb{L}^{4}}^{4} d s\right]\right)^{1 / 2} \\
& \quad \leq c_{4} \theta^{1 / 2} .
\end{aligned}
$$

Thus $J_{4}^{n}$ satisfies (5.2) with $\alpha=1$ and $\beta=\frac{1}{2}$.

Next consider the term $J_{5}^{n}(t)$. Consider the operator $C: L^{2}(\mathcal{O}) \rightarrow \mathbb{H}^{-1}(\mathcal{O})$ defined by $C(\hat{z})=$ $g \nabla \hat{z}$. For all $v \in \mathbb{H}_{0}^{1}(\mathcal{O})$, we have $|\langle C(\hat{z}), v\rangle|=\left|-g(\hat{z}, \operatorname{Div}(v))_{L^{2}}\right| \leq g\|\hat{z}\|_{L^{2}}\|v\|_{\mathbb{H}_{0}^{1}}$. Hence

$$
\|C(\hat{z})\|_{\mathbb{H}^{-1}} \leq g\|\hat{z}\|_{L^{2}}
$$

So by using (5.12), Hölder's inequality and (4.6), we have

$$
\begin{aligned}
& \mathbb{E}\left[\left\|J_{5}^{n}\left(\tau_{n}+\theta\right)-J_{5}^{n}\left(\tau_{n}\right)\right\|_{\mathbb{H}^{-1}}\right]=\mathbb{E}\left[\left\|\int_{\tau_{n}}^{\tau_{n}+\theta} g \nabla \hat{z}^{n}(s) d s\right\|_{\mathbb{H}^{-1}}\right] \\
& \quad \leq g \mathbb{E}\left[\int_{\tau_{n}}^{\tau_{n}+\theta}\left\|\hat{z}^{n}(s)\right\|_{L^{2}} d s\right] \leq g \theta^{1 / 2}\left[\mathbb{E}\left(\int_{\tau_{n}}^{\tau_{n}+\theta}\left\|\hat{z}^{n}(s)\right\|_{L^{2}}^{2} d s\right)\right]^{1 / 2} \\
& \quad \leq g \theta^{1 / 2}\left[\mathbb{E}\left(\int_{0}^{T}\left\|\hat{z}^{n}(s)\right\|_{L^{2}}^{2} d s\right)\right]^{1 / 2} \leq g \theta^{1 / 2}\left[\mathbb{E}\left(T \sup _{0 \leq s \leq T}\left\|\hat{z}^{n}(s)\right\|_{L^{2}}^{2}\right)\right]^{1 / 2} \\
& \leq g \theta^{1 / 2} T^{1 / 2}\left(\mathbb{E}\left[\sup _{0 \leq s \leq T}\left\|\hat{z}^{n}(s)\right\|_{L^{2}}^{2}\right]\right)^{1 / 2} \leq c_{5} \theta^{1 / 2} .
\end{aligned}
$$


Thus $J_{5}^{n}$ satisfies (5.2) with $\alpha=1$ and $\beta=\frac{1}{2}$.

We next consider the term $J_{6}^{n}(t)$. Since $\mathbb{L}^{2}(\mathcal{O}) \hookrightarrow \mathbb{H}^{-1}(\mathcal{O})$ and $f \in L^{2}\left(\Omega ; L^{2}\left(0, T ; \mathbb{L}^{2}(\mathcal{O})\right)\right)$, by Hölder inequality, we have

$$
\begin{aligned}
& \mathbb{E}\left[\left\|J_{6}^{n}\left(\tau_{n}+\theta\right)-J_{6}^{n}\left(\tau_{n}\right)\right\|_{\mathbb{H}-1}\right]=\mathbb{E}\left[\left\|\int_{\tau_{n}}^{\tau_{n}+\theta} f(s) d s\right\|_{\mathbb{H}-1}\right] \\
& \leq \mathbb{E}\left[\int_{\tau_{n}}^{\tau_{n}+\theta}\|f(s)\|_{\mathbb{H}^{-1}} d s\right] \leq c \mathbb{E}\left[\int_{\tau_{n}}^{\tau_{n}+\theta}\|f(s)\|_{\mathbb{L}^{2}} d s\right] \\
& \quad \leq c \theta^{1 / 2}\left(\mathbb{E}\left[\int_{\tau_{n}}^{\tau_{n}+\theta}\|f(s)\|_{\mathbb{L}^{2}}^{2} d s\right]\right)^{1 / 2} \\
& \quad \leq c \theta^{1 / 2}\left(\mathbb{E}\left[\int_{0}^{T}\|f(s)\|_{\mathbb{L}^{2}}^{2} d s\right]\right)^{1 / 2} \leq c_{6} \theta^{1 / 2} .
\end{aligned}
$$

Thus $J_{6}^{n}$ satisfies (5.2) with $\alpha=1$ and $\beta=\frac{1}{2}$.

Now we consider the term $J_{7}^{n}(t)$. Using the embedding $\mathbb{L}^{2}(\mathcal{O}) \hookrightarrow \mathbb{H}^{-1}(\mathcal{O})$, Itô isometry, (4.18), Assumption 3.12 and inequality (4.6), we obtain

$$
\begin{aligned}
& \mathbb{E}\left[\left\|J_{7}^{n}\left(\tau_{n}+\theta\right)-J_{7}^{n}\left(\tau_{n}\right)\right\|_{\mathbb{H}^{-1}}^{2}\right]=\mathbb{E}\left[\left\|\int_{\tau_{n}}^{\tau_{n}+\theta} \sigma^{n}\left(s, u^{n}(s)\right) d W^{n}(s)\right\|_{\mathbb{H}^{-1}}^{2}\right] \\
& \quad \leq c \mathbb{E}\left[\left\|\int_{\tau_{n}}^{\tau_{n}+\theta} \sigma^{n}\left(s, u^{n}(s)\right) d W^{n}(s)\right\|_{\mathbb{L}^{2}}^{2}\right]=c \mathbb{E}\left[\int_{\tau_{n}}^{\tau_{n}+\theta}\left\|\sigma^{n}\left(s, u^{n}(s)\right)\right\|_{L_{Q}\left(\mathbb{L}^{2}, \mathbb{L}^{2}\right)}^{2} d s\right] \\
& \quad \leq c \mathbb{E}\left[\int_{\tau_{n}}^{\tau_{n}+\theta}\left\|\sigma\left(s, u^{n}(s)\right)\right\|_{L_{Q}\left(\mathbb{L}^{2}, \mathbb{L}^{2}\right)}^{2} d s\right] \leq c K \mathbb{E}\left[\int_{\tau_{n}}^{\tau_{n}+\theta}\left(1+\left\|u^{n}(s)\right\|_{\mathbb{L}^{2}}^{2}\right) d s\right] \\
& \quad \leq c K \theta\left(1+\mathbb{E}\left[\sup _{0 \leq s \leq T}\left\|u^{n}(s)\right\|_{\mathbb{L}^{2}}^{2}\right]\right) \leq c_{6} \theta .
\end{aligned}
$$

Thus $J_{7}^{n}$ satisfies (5.2) with $\alpha=2$ and $\beta=1$.

We finally consider the term $J_{8}^{n}(t)$. Using the embedding $\mathbb{L}^{2}(\mathcal{O}) \hookrightarrow \mathbb{H}^{-1}(\mathcal{O})$, Lévy-Itô isometry, (4.19), Assumption 3.12 and inequality (4.6), we obtain

$$
\begin{aligned}
\mathbb{E}\left[\left\|J_{8}^{n}\left(\tau_{n}+\theta\right)-J_{8}^{n}\left(\tau_{n}\right)\right\|_{\mathbb{H}^{-1}}^{2}\right] & =\mathbb{E}\left[\left\|\int_{\tau_{n}}^{\tau_{n}+\theta} \int_{Z} H^{n}\left(u^{n}(s-), z\right) \tilde{N}(d s, d z)\right\|_{\mathbb{H}^{-1}}^{2}\right] \\
& \leq c \mathbb{E}\left[\left\|\int_{\tau_{n}}^{\tau_{n}+\theta} \int_{Z} H^{n}\left(u^{n}(s-), z\right) \tilde{N}(d s, d z)\right\|_{\mathbb{L}^{2}}^{2}\right] \\
& =c \mathbb{E}\left[\int_{\tau_{n}}^{\tau_{n}+\theta} \int_{Z}\left\|H^{n}\left(u^{n}(s), z\right)\right\|_{\mathbb{L}^{2}}^{2} \lambda(d z) d s\right] \\
& \leq c \mathbb{E}\left[\int_{\tau_{n}}^{\tau_{n}+\theta} \int_{Z}\left\|H\left(u^{n}(s), z\right)\right\|_{\mathbb{L}^{2}}^{2} \lambda(d z) d s\right] \\
& \left.\leq c K \mathbb{E}\left[\int_{\tau_{n}}^{\tau_{n}+\theta}\left(1+\| u^{n}(s)\right) \|_{\mathbb{L}^{2}}^{2}\right) d s\right] \\
& \leq c K \theta\left(1+\mathbb{E}\left[\sup _{0 \leq s \leq T}\left\|u^{n}(s)\right\|_{\mathbb{L}^{2}}^{2}\right]\right) \leq c_{6} \theta .
\end{aligned}
$$

Thus $J_{8}^{n}$ satisfies (5.2) with $\alpha=2$ and $\beta=1$.

Finally combining estimates of each $J_{i}^{n} ; i=1, \cdots, 8$, we have,

$$
\begin{aligned}
& \mathbb{E}\left[\left\|u^{n}\left(\tau_{n}+\theta\right)-u^{n}\left(\tau_{n}\right)\right\|_{\mathbb{H}^{-1}}\right]=\mathbb{E}\left[\| \sum_{i=1}^{8}\left(J_{i}^{n}\left(\tau_{n}+\theta\right)-J_{i}^{n}\left(\tau_{n}\right) \|_{\mathbb{H}^{-1}}\right]\right. \\
& \leq \sum_{i=1}^{8} \mathbb{E}\left[\|\left(J_{i}^{n}\left(\tau_{n}+\theta\right)-J_{i}^{n}\left(\tau_{n}\right) \|_{\mathbb{H}^{-1}}\right]=\sum_{i=1}^{6} \mathbb{E}\left[\|\left(J_{i}^{n}\left(\tau_{n}+\theta\right)-J_{i}^{n}\left(\tau_{n}\right) \|_{\mathbb{H}^{-1}}\right]\right.\right.
\end{aligned}
$$




$$
\begin{aligned}
& +\sum_{i=7}^{8} \mathbb{E}\left[\|\left(J_{i}^{n}\left(\tau_{n}+\theta\right)-J_{i}^{n}\left(\tau_{n}\right) \|_{\mathbb{H}^{-1}}\right]\right. \\
\leq & C_{1} \theta^{1 / 2}+\sum_{i=7}^{8}\left[\mathbb { E } \left(\|\left(J_{i}^{n}\left(\tau_{n}+\theta\right)-J_{i}^{n}\left(\tau_{n}\right) \|_{\mathbb{H}^{-1}}^{2}\right]^{1 / 2}\right.\right. \\
\leq & C_{1} \theta^{1 / 2}+C_{2} \theta^{1 / 2}:=C \theta^{1 / 2}
\end{aligned}
$$

Hence $u^{n}$ satisfies Aldous condition in the space $\mathbb{H}^{-1}(\mathcal{O})$ with $\alpha=1$ and $\beta=\frac{1}{2}$, which completes the proof.

Lemma 5.11. The set of measures $\left\{\mathcal{L}\left(\hat{z}^{n}\right), n \in \mathbb{N}\right\}$ is tight on $L^{2}\left(0, T ; L^{2}(\mathcal{O})\right) \cap C\left([0, T] ; H^{-1}(\mathcal{O})\right)$.

Proof. First note that, due to lack of $H^{1}$ estimate for $\hat{z}^{n}$, we can not apply Theorem 5.8 , and hence we may not be able prove tightness following the method of the previous Lemma. However, one could possibly consider more regular initial data and apply Proposition A.1 to obtain $H^{1}$ estimate for $\hat{z}^{n}$ and then apply Theorem 5.8 to obtain tightness. But we do not proceed in this direction. To prove the tightness in $L^{2}\left(0, T ; L^{2}(\mathcal{O})\right)$, we follow the classical argument of Chow and Khasminskii [16].

By Proposition 4.3, $\mathbb{E}\left[\sup _{0 \leq t \leq T}\left\|\hat{z}^{n}(t)\right\|_{L^{2}}^{2}\right] \leq C_{1}$, hence using Fubini's theorem we have

$$
\mathbb{E}\left[\left\|\hat{z}^{n}\right\|_{L^{2}\left(0, T ; L^{2}\right)}^{2}\right] \leq C_{1} T .
$$

By the Chebychev inequality, we see that for any $r>0$

$$
P\left(\left\|\hat{z}^{n}\right\|_{L^{2}\left(0, T ; L^{2}\right)}>r\right) \leq \frac{\mathbb{E}\left[\left\|\hat{z}^{n}\right\|_{L^{2}\left(0, T ; L^{2}\right)}^{2}\right]}{r^{2}} \leq \frac{C_{1} T}{r^{2}} .
$$

Let $\epsilon>0$ be given. Let $R_{1}>0$ be such that $\frac{C_{1} T}{R_{1}^{2}}<\epsilon$. Then $P\left(\left\|\hat{z}^{n}\right\|_{L^{2}\left(0, T ; L^{2}\right)}>R_{1}\right)<\epsilon$. Denote

$$
B_{\epsilon}:=\left\{\hat{z}^{n} \in L^{2}\left(0, T ; L^{2}(\mathcal{O})\right):\left\|\hat{z}^{n}\right\|_{L^{2}\left(0, T ; L^{2}\right)} \leq R_{1}\right\} .
$$

Then it is clear that $P\left(B_{\epsilon}\right) \geq 1-\epsilon$. Hence for every $\epsilon>0$, there exists a compact subset $B_{\epsilon}$ of $L^{2}\left(0, T ; L^{2}(\mathcal{O})\right)$ such that $P\left(B_{\epsilon}\right) \geq 1-\epsilon$. Thus the set of measures $\left\{\mathcal{L}\left(\hat{z}^{n}\right), n \in \mathbb{N}\right\}$ is tight on $L^{2}\left(0, T ; L^{2}(\mathcal{O})\right)$.

To prove the tightness in $C\left([0, T] ; H^{-1}(\mathcal{O})\right)$, we follow the method due to Flandoli and Gatarek 19] (see Theorem 3.1, Step 2).

We decompose $\hat{z}^{n}$ as

$$
\begin{aligned}
\hat{z}^{n}(t) & =\hat{z}_{0}^{n}-\int_{0}^{t} \operatorname{Div}\left(h u^{n}(s)\right) d s \\
& =J_{9}^{n}+J_{10}^{n}(t) .
\end{aligned}
$$

We already have $\mathbb{E}\left\|J_{9}^{n}\right\|_{L^{2}}^{2} \leq C_{1}$. Also, by Proposition 4.3 we have

$$
\begin{aligned}
& \mathbb{E}\left\|J_{10}^{n}(t)\right\|_{L^{2}\left(0, T ; L^{2}\right)}^{2} \leq \mathbb{E} \int_{0}^{T} \int_{0}^{t}\left\|\operatorname{Div}\left(h u^{n}(s)\right)\right\|_{L^{2}}^{2} d s d t \\
& \leq 2 T \mathbb{E} \int_{0}^{T}\left(\|\nabla h\|_{\mathbb{L}^{\infty}}^{2}\left\|u^{n}(t)\right\|_{\mathbb{L}^{2}}^{2}+\|h\|_{L^{\infty}}^{2}\left\|\nabla u^{n}(t)\right\|_{\mathbb{L}^{2}}^{2}\right) d t \\
& \leq 2 T^{2}\|\nabla h\|_{\mathbb{L}^{\infty}}^{2} \mathbb{E}\left[\sup _{0 \leq t \leq T}\left\|u^{n}(t)\right\|_{\mathbb{L}^{2}}^{2}\right]+2 T\|h\|_{L^{\infty}}^{2} \mathbb{E} \int_{0}^{T}\left\|\nabla u^{n}(t)\right\|_{\mathbb{L}^{2}}^{2} d t \\
& \leq C_{2} .
\end{aligned}
$$

Thus we have $\mathbb{E}\left\|\hat{z}^{n}\right\|_{L^{2}\left(0, T ; L^{2}\right)}^{2} \leq C_{3}$, and hence $\mathbb{E}\left\|\hat{z}^{n}\right\|_{W^{1,2}\left(0, T ; L^{2}\right)}^{2} \leq C_{4}$, where for a generic Banach space $B$ and a real number $p \geq 1, W^{1, p}(0, T ; B)$ denotes the space of all $v \in L^{p}(0, T ; B)$ such that $\frac{d v}{d t} \in L^{p}(0, T ; B)$. Since $W^{1,2}\left(0, T ; L^{2}(\mathcal{O})\right) \subset W^{\alpha, 2}\left(0, T ; L^{2}(\mathcal{O})\right)$ for all $\alpha \in(0,1)$, we infer

$$
\mathbb{E}\left\|\hat{z}^{n}\right\|_{W^{\alpha, 2}\left(0, T ; L^{2}\right)}^{2} \leq C_{4}
$$


Since $L^{2}(\mathcal{O})$ is compactly embedded in $H^{-1}(\mathcal{O})$, we can apply Theorem 2.2 of Flandoli and Gatarek [19] to infer that for all real numbers $\alpha \in\left(\frac{1}{2}, 1\right)$, the space $W^{\alpha, 2}\left(0, T ; L^{2}(\mathcal{O})\right)$ is compactly embedded into $C\left([0, T] ; H^{-1}(\mathcal{O})\right)$. Hence the set of measures $\left\{\mathcal{L}\left(\hat{z}^{n}\right), n \in \mathbb{N}\right\}$ is tight on $C\left([0, T] ; H^{-1}(\mathcal{O})\right)$.

Lemma 5.12. The set of measures $\left\{\mathcal{L}\left(U^{n}\right), n \in \mathbb{N}\right\}$ is tight on $\mathbb{L}^{2}(\mathcal{O})$.

Proof. Using the assumption $\mathbb{E}\left[\left\|U^{n}\right\|_{\mathbb{L}^{2}}^{2}\right] \leq c$, and using the Chebychev inequality, we see that for any $r>0$

$$
P\left(\left\|U^{n}\right\|_{\mathbb{L}^{2}}>r\right) \leq \frac{\mathbb{E}\left[\left\|U^{n}\right\|_{\mathbb{L}^{2}}^{2}\right]}{r^{2}} \leq \frac{c}{r^{2}}
$$

Let $\epsilon>0$ be given. Let $R_{2}>0$ be such that $\frac{c}{R_{2}^{2}}<\epsilon$. Then

$$
P\left(\left\|U^{n}\right\|_{\mathbb{L}^{2}}>R_{2}\right)<\epsilon .
$$

Denote

$$
B_{\epsilon}=\left\{U^{n} \in \mathbb{L}^{2}(\mathcal{O}):\left\|U^{n}\right\|_{\mathbb{L}^{2}} \leq R_{2}\right\} .
$$

Then $P\left(B_{\epsilon}\right) \geq 1-\epsilon$. Hence for every $\epsilon>0$, there exists a compact subset $B_{\epsilon}$ of $\mathbb{L}^{2}(\mathcal{O})$ such that $P\left(B_{\epsilon}\right) \geq 1-\epsilon$. This proves the Lemma.

Theorem 5.13. There exists a martingale solution of (5.4)-(5.6) provided the Assumptions 3.12 are satisfied.

\section{Proof. Step I:}

By Lemmas [5.10, 5.11 and 5.12, the set of measures $\left\{\mathcal{L}\left(u^{n}\right), n \in \mathbb{N}\right\},\left\{\mathcal{L}\left(\hat{z}^{n}\right), n \in \mathbb{N}\right\}$ and $\left\{\mathcal{L}\left(U^{n}\right), n \in \mathbb{N}\right\}$ are tight on the spaces $(\mathcal{Z}, \tau), L^{2}\left(0, T ; L^{2}(\mathcal{O})\right) \cap C\left([0, T] ; H^{-1}(\mathcal{O})\right)$, and $\mathbb{L}^{2}(\mathcal{O})$ respectively. Define $N^{n}=N, \forall n \in \mathbb{N}$. Then the set of measures $\left\{\mathcal{L}\left(N^{n}\right), n \in \mathbb{N}\right\}$ is tight on the space $M_{\overline{\mathbb{N}}}([0, T] \times Z)$, where $\overline{\mathbb{N}}:=\mathbb{N} \cup\{\infty\}$ and $M_{\overline{\mathbb{N}}}(S)$ denotes the set of all $\overline{\mathbb{N}}$-valued measures on the measurable space $(S, \mathscr{B}(S))$ (see Motyl [37, [38] for more details). Define $W^{n}=W, \forall n \in \mathbb{N}$. Then the set of measures $\left\{\mathcal{L}\left(W^{n}\right), n \in \mathbb{N}\right\}$ is tight on the space $C([0, T] ; \mathbb{R})$ of continuous function from $[0, T]$ to $\mathbb{R}$ with standard supremum norm.

Thus the set $\left\{\mathcal{L}\left(u^{n}, \hat{z}^{n}, U^{n}, N^{n}, W^{n}\right), n \in \mathbb{N}\right\}$ is tight on $\mathcal{Z} \times\left(L^{2}\left(0, T ; L^{2}(\mathcal{O})\right) \cap C\left([0, T] ; H^{-1}(\mathcal{O})\right)\right) \times$ $\mathbb{L}^{2}(\mathcal{O}) \times M_{\overline{\mathbb{N}}}([0, T] \times Z) \times C([0, T] ; \mathbb{R})$. By the Skorokhod theorem [38, there exists a subsequence $\left(n_{k}\right)_{k \in \mathbb{N}}$, a probability space $(\bar{\Omega}, \overline{\mathcal{F}}, \bar{P})$, and, on this space, random variables $\left(u^{*}, z^{*}, U^{*}, N^{*}, W^{*}\right)$, $\left\{\left(\bar{u}^{k}, \bar{z}^{k}, \bar{U}^{k}, \bar{N}^{k}, \bar{W}^{k}\right), k \in \mathbb{N}\right\}$ such that

(i) $\mathcal{L}\left(\left(\bar{u}^{k}, \bar{z}^{k}, \bar{U}^{k}, \bar{N}^{k}, \bar{W}^{k}\right)\right)=\mathcal{L}\left(\left(u^{n_{k}}, \hat{z}^{n_{k}}, U^{n_{k}}, N^{n_{k}}, W^{n_{k}}\right)\right)$ for all $k \in \mathbb{N}$,

(ii) $\left(\bar{u}^{k}, \bar{z}^{k}, \bar{U}^{k}, \bar{N}^{k}, \bar{W}^{k}\right) \rightarrow\left(u^{*}, z^{*}, U^{*}, N^{*}, W^{*}\right)$ in $\mathcal{Z} \times\left(L^{2}\left(0, T ; L^{2}(\mathcal{O})\right) \cap C\left([0, T] ; H^{-1}(\mathcal{O})\right)\right) \times$ $\mathbb{L}^{2}(\mathcal{O}) \times M_{\overline{\mathbb{N}}}([0, T] \times Z) \times C([0, T] ; \mathbb{R})$ with probability 1 on $(\bar{\Omega}, \overline{\mathcal{F}}, \bar{P})$ as $k \rightarrow \infty$,

(iii) $\left(\bar{N}^{k}(\bar{\omega}), \bar{W}^{k}(\bar{\omega})\right)=\left(N^{*}(\bar{\omega}), W^{*}(\bar{\omega})\right)$ for all $\bar{\omega} \in \bar{\Omega}$.

We denote these sequences again by $\left(\left(u^{n}, \hat{z}^{n}, U^{n}, N^{n}, W^{n}\right)\right)_{n \in \mathbb{N}}$ and $\left(\left(\bar{u}^{n}, \bar{z}^{n}, \bar{U}^{n}, \bar{N}^{n}, \bar{W}^{n}\right)\right)_{n \in \mathbb{N}}$. Using the definition of the space $\mathcal{Z}$, we have $\bar{P}-$ a.s.

$$
\begin{gathered}
\bar{u}^{n} \rightarrow u^{*} \text { in } L_{w}^{2}\left(0, T ; \mathbb{H}_{0}^{1}(\mathcal{O})\right) \cap L^{2}\left(0, T ; \mathbb{L}^{2}(\mathcal{O})\right) \cap D\left([0, T] ; \mathbb{H}^{-1}(\mathcal{O})\right) \\
\cap D\left([0, T] ; \mathbb{L}_{w}^{2}(\mathcal{O})\right), \\
\bar{z}^{n} \rightarrow z^{*} \quad \text { in } \quad L^{2}\left(0, T ; L^{2}(\mathcal{O})\right) \cap C\left([0, T] ; H^{-1}(\mathcal{O})\right),
\end{gathered}
$$

and

$$
\bar{U}^{n} \rightarrow U^{*} \text { in } \mathbb{L}^{2}(\mathcal{O})
$$

Note that, since $D\left([0, T] ; \mathbb{L}_{n}^{2}(\mathcal{O})\right)$ is a Polish space and $L_{w}^{2}\left(0, T ; \mathbb{H}_{0}^{1}(\mathcal{O})\right) \cap L^{2}\left(0, T ; \mathbb{L}^{2}(\mathcal{O})\right) \cap$ $D\left([0, T] ; \mathbb{H}^{-1}(\mathcal{O})\right) \cap D\left([0, T] ; \mathbb{L}_{w}^{2}(\mathcal{O})\right)$ is a separable metric space, due to Kuratowski theorem (see Theorem 1.1, Chapter 1 of [49]), Borel subsets of $D\left([0, T] ; \mathbb{L}_{n}^{2}(\mathcal{O})\right.$ ) are Borel subsets of $L_{w}^{2}\left(0, T ; \mathbb{H}_{0}^{1}(\mathcal{O})\right) \cap L^{2}\left(0, T ; \mathbb{L}^{2}(\mathcal{O})\right) \cap D\left([0, T] ; \mathbb{H}^{-1}(\mathcal{O})\right) \cap D\left([0, T] ; \mathbb{L}_{w}^{2}(\mathcal{O})\right)$, and $P\left\{u^{n} \in D([0, T]\right.$ 
$\left.\left.\mathbb{L}_{n}^{2}(\mathcal{O})\right)\right\}=1$. Hence, we may assume that $\bar{u}^{n}$ takes values in $\mathbb{L}_{n}^{2}(\mathcal{O})$ and that the laws on $D\left([0, T] ; \mathbb{L}_{n}^{2}(\mathcal{O})\right)$ of $u^{n}$ and $\bar{u}^{n}$ are equal.

In view of the above, it is straightforward to show that the sequence $\left(\bar{u}^{n}\right)_{n \in \mathbb{N}}$ satisfies the same estimates as the original sequence $\left(u^{n}\right)_{n \in \mathbb{N}}$. In particular, for any $p \geq 2$, we have

$$
\sup _{n \geq 1} \overline{\mathbb{E}}\left[\sup _{0 \leq s \leq T}\left\|\bar{u}^{n}(s)\right\|_{\mathbb{L}^{2}}^{p}\right] \leq C_{1(p)}
$$

and

$$
\sup _{n \geq 1} \overline{\mathbb{E}}\left[\int_{0}^{T}\left\|\bar{u}^{n}(s)\right\|_{\mathbb{H}_{0}^{1}}^{2} d s\right] \leq C_{2(2)} .
$$

Again repeating the same arguments (as above) for the random variables $\bar{z}^{n}$ and $\hat{z}^{n}$, it is obvious to show that the sequence $\left(\bar{z}^{n}\right)_{n \in \mathbb{N}}$ satisfies the same estimates as the original sequence $\left(\hat{z}^{n}\right)_{n \in \mathbb{N}}$. Hence we have,

$$
\sup _{n \geq 1} \overline{\mathbb{E}}\left[\sup _{0 \leq s \leq T}\left\|\bar{z}^{n}(s)\right\|_{L^{2}}^{2}\right] \leq C_{1(2)} .
$$

Now, using the assumption $\mathbb{E}\left[\|U\|_{\mathbb{L}^{2}}^{2}\right] \leq c$, we have

$$
\sup _{n \geq 1} \overline{\mathbb{E}}\left[\left\|\bar{U}^{n}\right\|_{\mathbb{L}^{2}}^{2}\right] \leq c .
$$

Proposition 5.14. Let $u^{*}$ be the limiting process defined above. Then

$$
\overline{\mathbb{E}}\left[\int_{0}^{T}\left\|u^{*}(s)\right\|_{\mathbb{H}_{0}^{1}}^{2} d s\right] \leq C,
$$

and for $r \geq 2$,

$$
\overline{\mathbb{E}}\left[\sup _{s \in[0, T]}\left\|u^{*}(s)\right\|_{\mathbb{L}^{2}}^{r}\right] \leq C_{r} .
$$

Proof. We begin the proof by establishing our Claim.

Claim 3. $\bar{u}^{n} \stackrel{w}{\rightarrow} u^{*}$ in $L^{2}\left(\bar{\Omega} ; L^{2}\left(0, T ; \mathbb{H}_{0}^{1}(\mathcal{O})\right)\right)$, i.e.,

$$
\begin{aligned}
\overline{\mathbb{E}}\left[\int_{0}^{T}\left\langle\bar{u}^{n}(t, \omega), \phi(t, \omega)\right\rangle d t\right] & \rightarrow \overline{\mathbb{E}}\left[\int_{0}^{T}\left\langle u^{*}(t, \omega), \phi(t, \omega)\right\rangle d t\right] \\
\forall \phi & \in L^{2}\left(\bar{\Omega} ; L^{2}\left(0, T ; \mathbb{H}^{-1}(\mathcal{O})\right)\right) .
\end{aligned}
$$

\section{Proof of Claim 3.}

Let $1<s<2$. Then $\frac{2 s}{2-s}>2$. Let $\phi \in L^{\frac{2 s}{2-s}}\left(\bar{\Omega} ; L^{2}\left(0, T ; \mathbb{H}^{-1}(\mathcal{O})\right)\right)$. Then $\phi(\cdot, \omega) \in$ $\left.L^{2}\left(0, T ; \mathbb{H}^{-1}(\mathcal{O})\right)\right) \bar{P}-$ a.s.

By (5.14) we have $\bar{u}^{n} \rightarrow u^{*}$ in $L_{w}^{2}\left(0, T ; \mathbb{H}_{0}^{1}(\mathcal{O})\right) \bar{P}-$ a.s. Hence,

$$
\int_{0}^{T}\left\langle\bar{u}^{n}(t, \omega), \phi(t, \omega)\right\rangle d t \rightarrow \int_{0}^{T}\left\langle u^{*}(t, \omega), \phi(t, \omega)\right\rangle d t \bar{P}-\text { a.s. }
$$

We note that by Hölder's inequality with $\frac{1}{2}+\frac{1}{2}=1$ and $\frac{s}{2}+\frac{2-s}{2}=1$ and using (5.18) we achieve

$$
\begin{aligned}
& \overline{\mathbb{E}}\left[\left|\int_{0}^{T}\left\langle\bar{u}^{n}(t, \omega), \phi(t, \omega)\right\rangle d t\right|^{s}\right] \leq \overline{\mathbb{E}}\left[\left(\int_{0}^{T}\left|\left\langle\bar{u}^{n}(t, \omega), \phi(t, \omega)\right\rangle\right| d t\right)^{s}\right] \\
& \leq \overline{\mathbb{E}}\left[\left(\int_{0}^{T} \mid\left\|\bar{u}^{n}(t)\right\|_{\mathbb{H}_{0}^{1}}\|\phi(t)\|_{\mathbb{H}^{-1}} d t\right)^{s}\right] \\
& \leq \overline{\mathbb{E}}\left[\left(\int_{0}^{T} \mid\left\|\bar{u}^{n}(t)\right\|_{\mathbb{H}_{0}^{1}}^{2} d t\right)^{\frac{s}{2}}\left(\int_{0}^{T}\|\phi(t)\|_{\mathbb{H}^{-1}}^{2} d t\right)^{\frac{s}{2}}\right] \\
& \leq\left[\overline{\mathbb{E}}\left(\int_{0}^{T} \mid\left\|\bar{u}^{n}(t)\right\|_{\mathbb{H}_{0}^{1}}^{2} d t\right)\right]^{\frac{s}{2}}\left[\overline{\mathbb{E}}\left(\int_{0}^{T}\|\phi(t)\|_{\mathbb{H}^{-1}}^{2} d t\right)^{\frac{s}{2-s}}\right]^{\frac{2-s}{2}} \\
& \leq C\left[\overline{\mathbb{E}}\left(\|\phi\|_{L^{2}\left(0, T ; \mathbb{H}^{-1}\right)}^{\frac{2 s}{2-s}}\right)\right]^{\frac{2-s}{2}} \leq C .
\end{aligned}
$$


Using (5.24), (5.25) and by Vitali Theorem we have

$$
\begin{aligned}
\overline{\mathbb{E}}\left[\int_{0}^{T}\left\langle\bar{u}^{n}(t, \omega), \phi(t, \omega)\right\rangle d t\right] & \rightarrow \overline{\mathbb{E}}\left[\int_{0}^{T}\left\langle u^{*}(t, \omega), \phi(t, \omega)\right\rangle d t\right] \\
\forall \phi & \in L^{\frac{2 s}{2-s}}\left(\bar{\Omega} ; L^{2}\left(0, T ; \mathbb{H}^{-1}(\mathcal{O})\right)\right) .
\end{aligned}
$$

As $1<s<2$, so $\frac{2 s}{2-s}>2$. Hence $L^{\frac{2 s}{2-s}}\left(\bar{\Omega} ; L^{2}\left(0, T ; \mathbb{H}^{-1}(\mathcal{O})\right)\right)$ is dense in $L^{2}\left(\bar{\Omega} ; L^{2}\left(0, T ; \mathbb{H}^{-1}(\mathcal{O})\right)\right)$. Therefore,

$$
\begin{aligned}
\overline{\mathbb{E}}\left[\int_{0}^{T}\left\langle\bar{u}^{n}(t, \omega), \phi(t, \omega)\right\rangle d t\right] & \rightarrow \overline{\mathbb{E}}\left[\int_{0}^{T}\left\langle u^{*}(t, \omega), \phi(t, \omega)\right\rangle d t\right] \\
\forall \phi & \in L^{2}\left(\bar{\Omega} ; L^{2}\left(0, T ; \mathbb{H}^{-1}(\mathcal{O})\right)\right) .
\end{aligned}
$$

This proves our claim.

Using Claim 3 it can be directly observed that $u^{*} \in L^{2}\left(\bar{\Omega} ; L^{2}\left(0, T ; \mathbb{H}_{0}^{1}(\mathcal{O})\right)\right.$, i.e., (5.21) is established.

Now we will prove (5.22). By (5.17) we have that $\left\{\bar{u}^{n}\right\}_{n \geq 1}$ is uniformly bounded in $L^{r}(\bar{\Omega}$;

$\left.L^{\infty}\left(0, T ; \mathbb{L}^{2}(\mathcal{O})\right)\right)$ for $r \geq 2$. Since $L^{r}\left(\bar{\Omega} ; L^{\infty}\left(0, T ; \mathbb{L}^{2}(\mathcal{O})\right)\right)$ is isomorphic to the space $\left(L^{\frac{r}{r-1}}(\bar{\Omega}\right.$; $\left.\left.L^{1}\left(0, T ; \mathbb{L}^{2}(\mathcal{O})\right)\right)\right)^{*}$, by Banach Alaoglu Theorem, there exists a subsequence still denoted by $\left\{\bar{u}^{n}\right\}_{n \geq 1}$ and $\zeta \in L^{r}\left(\bar{\Omega} ; L^{\infty}\left(0, T ; \mathbb{L}^{2}(\mathcal{O})\right)\right)$ such that

$$
\bar{u}^{n} \stackrel{w^{*}}{\longrightarrow} \zeta \quad \text { in } \quad L^{r}\left(\bar{\Omega} ; L^{\infty}\left(0, T ; \mathbb{L}^{2}(\mathcal{O})\right)\right)
$$

i.e.,

$$
\begin{aligned}
\overline{\mathbb{E}}\left[\int_{0}^{T}\left(\bar{u}^{n}(t, \omega), \phi(t, \omega)\right)_{\mathbb{L}^{2}} d t\right] & \rightarrow \overline{\mathbb{E}}\left[\int_{0}^{T}(\zeta(t, \omega), \phi(t, \omega))_{\mathbb{L}^{2}} d t\right] \\
\forall \phi & \in L^{\frac{r}{r-1}}\left(\bar{\Omega} ; L^{1}\left(0, T ; \mathbb{L}^{2}(\mathcal{O})\right)\right) .
\end{aligned}
$$

Employing Claim 3 and Gelfand triple $\mathbb{H}_{0}^{1}(\mathcal{O}) \subset \mathbb{L}^{2}(\mathcal{O}) \subset \mathbb{H}^{-1}(\mathcal{O})$ we have

$$
\begin{aligned}
\overline{\mathbb{E}}\left[\int_{0}^{T}\left(\bar{u}^{n}(t, \omega), \phi(t, \omega)\right)_{\mathbb{L}^{2}} d t\right] & \rightarrow \overline{\mathbb{E}}\left[\int_{0}^{T}\left(u^{*}(t, \omega), \phi(t, \omega)\right)_{\mathbb{L}^{2}} d t\right] \\
& \forall \phi \in L^{2}\left(\bar{\Omega} ; L^{2}\left(0, T ; \mathbb{L}^{2}(\mathcal{O})\right)\right) .
\end{aligned}
$$

Again for $r \geq 2, L^{2}\left(\bar{\Omega} ; L^{2}\left(0, T ; \mathbb{L}^{2}(\mathcal{O})\right)\right)$ is dense subspace of $L^{\frac{r}{r-1}}\left(\bar{\Omega} ; L^{1}\left(0, T ; \mathbb{L}^{2}(\mathcal{O})\right)\right)$. (5.27) and (5.28) jointly produces

$$
\begin{aligned}
\overline{\mathbb{E}}\left[\int_{0}^{T}(\zeta(t, \omega), \phi(t, \omega))_{\mathbb{L}^{2}} d t\right] & =\overline{\mathbb{E}}\left[\int_{0}^{T}\left(u^{*}(t, \omega), \phi(t, \omega)\right)_{\mathbb{L}^{2}} d t\right] \\
& \forall \phi \in L^{2}\left(\bar{\Omega} ; L^{2}\left(0, T ; \mathbb{L}^{2}(\mathcal{O})\right)\right) .
\end{aligned}
$$

Thus we have, $u^{*}(t, \omega)=\zeta(t, \omega)$ for almost every $t \in[0, T]$ and $\omega \in \bar{\Omega}$. Since $\zeta \in L^{r}\left(\bar{\Omega} ; L^{\infty}\left(0, T ; \mathbb{L}^{2}(\mathcal{O})\right)\right)$, we infer that $u^{*} \in L^{r}\left(\bar{\Omega} ; L^{\infty}\left(0, T ; \mathbb{L}^{2}(\mathcal{O})\right)\right)$, i.e.,

$$
\overline{\mathbb{E}}\left[\sup _{s \in[0, T]}\left\|u^{*}(s)\right\|_{\mathbb{L}^{2}}^{r}\right] \leq C_{r}
$$

for some constant $C_{r}$ (depending on $\mathrm{r}$ ).

\section{Step II:}

Lemma 5.15. For all $v \in \mathbb{H}_{0}^{1}(\mathcal{O})$ and for all $w \in H_{0}^{1}(\mathcal{O})$,

(i) $\lim _{n \rightarrow \infty} \overline{\mathbb{E}}\left[\int_{0}^{T}\left|\left(\bar{u}^{n}(t)-u^{*}(t), v\right)_{\mathbb{L}^{2}}\right| d t\right]=0$.

(ii) $\lim _{n \rightarrow \infty} \overline{\mathbb{E}}\left[\left|\left(\bar{u}^{n}(0)-u^{*}(0), v\right)_{\mathbb{L}^{2}}\right|\right]=0$.

(ii) $\lim _{n \rightarrow \infty} \overline{\mathbb{E}}\left[\left|\left(\bar{U}^{n}-U^{*}, v\right)_{\mathbb{L}^{2}}\right|\right]=0$.

(iv) $\lim _{n \rightarrow \infty} \overline{\mathbb{E}}\left[\int_{0}^{T}\left|\int_{0}^{t}\left(A \bar{u}^{n}(s)-A u^{*}(s), v\right)_{\mathbb{L}^{2}} d s\right| d t\right]=0$.

(v) $\lim _{n \rightarrow \infty} \overline{\mathbb{E}}\left[\int_{0}^{T}\left|\int_{0}^{t}\left(B\left(\bar{u}^{n}(s)\right)-B\left(u^{*}(s)\right), v\right)_{\mathbb{L}^{2}} d s\right| d t\right]=0$.

(vi) $\lim _{n \rightarrow \infty} \overline{\mathbb{E}}\left[\int_{0}^{T}\left|\int_{0}^{t}\left\langle g \nabla\left(\bar{z}^{n}(s)-z^{*}(s)\right), v\right\rangle d s\right| d t\right]=0$. 
(vii) $\lim _{n \rightarrow \infty} \overline{\mathbb{E}}\left[\int_{0}^{T}\left\|\left(\int_{0}^{t}\left(\sigma^{n}\left(s, \bar{u}^{n}(s)\right)-\sigma\left(s, u^{*}(s)\right)\right) d W^{*}(s), v\right)_{\mathbb{L}^{2}}\right\|_{L_{Q}\left(\mathbb{L}^{2} ; \mathbb{R}\right)}^{2}\right] d t=0$.

(viii) $\lim _{n \rightarrow \infty} \overline{\mathbb{E}}\left[\int_{0}^{T}\left|\int_{0}^{t} \int_{Z}\left(H^{n}\left(\bar{u}^{n}(s), z\right)-H\left(u^{*}(s), z\right), v\right)_{\mathbb{L}^{2}} \lambda(d z) d s\right|^{2} d t\right]=0$.

(ix) $\lim _{n \rightarrow \infty} \overline{\mathbb{E}}\left[\int_{0}^{T}\left|\int_{0}^{t} \int_{Z}\left(H^{n}\left(\bar{u}^{n}(s-), z\right)-H\left(u^{*}(s-), z\right), v\right)_{\mathbb{L}^{2}} \tilde{N}^{*}(d s, d z)\right|^{2} d t\right]=0$.

(x) $\lim _{n \rightarrow \infty} \overline{\mathbb{E}}\left[\int_{0}^{T}\left|\left(\bar{z}^{n}(t)-z^{*}(t), w\right)_{L^{2}}\right| d t\right]=0$.

(xi) $\lim _{n \rightarrow \infty} \overline{\mathbb{E}}\left[\left|\left(\bar{z}_{0}^{n}-z_{0}^{*}, w\right)_{L^{2}}\right|\right]=0$.

(xii) $\lim _{n \rightarrow \infty} \overline{\mathbb{E}}\left[\int_{0}^{T}\left|\int_{0}^{t}\left(\operatorname{Div}\left(h \bar{u}^{n}(s)\right)-\operatorname{Div}\left(h u^{*}(s)\right), w\right)_{L^{2}} d s\right| d t\right]=0$.

Proof. Let $v \in \mathbb{H}_{0}^{1}(\mathcal{O})$ and $w \in H_{0}^{1}(\mathcal{O})$ be fixed.

(i) We have $\bar{u}^{n} \rightarrow u^{*}$ in $D\left([0, T] ; \mathbb{L}_{w}^{2}(\mathcal{O})\right)$, $\bar{P}$-a.s. i.e., $\left(\bar{u}^{n}(t)-u^{*}(t), v\right)_{\mathbb{L}^{2}} \rightarrow 0$ in $D([0, T] ; \mathbb{R}), \bar{P}-$ a.s. Hence, in particular for almost all $t \in[0, T]$,

$$
\lim _{n \rightarrow \infty}\left(\bar{u}^{n}(t), v\right)_{\mathbb{L}^{2}}=\left(u^{*}(t), v\right)_{\mathbb{L}^{2}} \quad \bar{P}-\text { a.s. }
$$

Employing Hölder's inequality and (5.17) we obtain

$$
\begin{aligned}
& \overline{\mathbb{E}}\left[\int_{0}^{T}\left|\left(\bar{u}^{n}(t)-u^{*}(t), v\right)_{\mathbb{L}^{2}}\right|^{2} d t\right] \leq \overline{\mathbb{E}}\left[\int_{0}^{T} \mid\left\|\bar{u}^{n}(t)-u^{*}(t)\right\|_{\mathbb{L}^{2}}^{2}\|v\|_{\mathbb{L}^{2}}^{2} d t\right] \\
& \leq\|v\|_{\mathbb{L}^{2}}^{2} \overline{\mathbb{E}}\left[\int_{0}^{T} \mid\left\|\bar{u}^{n}(t)-u^{*}(t)\right\|_{\mathbb{L}^{2}}^{2} d t\right] \leq 2\|v\|_{\mathbb{L}^{2}}^{2} \overline{\mathbb{E}}\left[\int_{0}^{T}\left(\left\|\bar{u}^{n}(t)\right\|_{\mathbb{L}^{2}}^{2}+\left\|u^{*}(t)\right\|_{\mathbb{L}^{2}}^{2}\right) d t\right] \\
& \leq 2 c T\|v\|_{\mathbb{H}_{0}^{1}}^{2} \overline{\mathbb{E}}\left[\sup _{0 \leq s \leq T}\left(\left\|\bar{u}^{n}(s)\right\|_{\mathbb{L}^{2}}^{2}+\left\|u^{*}(s)\right\|_{\mathbb{L}^{2}}^{2}\right)\right] \leq C .
\end{aligned}
$$

Hence, by employing (5.29), (5.30) and by Vitali Theorem we have assertion (i).

(ii) We have $\bar{u}^{n} \rightarrow u^{*}$ in $D\left([0, T] ; \mathbb{L}_{w}^{2}(\mathcal{O})\right), \bar{P}$-a.s. and $u^{*}$ is right continuous at $t=0$, we infer that

$$
\left(\bar{u}^{n}(0), v\right)_{\mathbb{L}^{2}} \rightarrow\left(u^{*}(0), v\right)_{\mathbb{L}^{2}} \bar{P}-\text { a.s. }
$$

Using (5.17),

$$
\overline{\mathbb{E}}\left[\left|\left(\bar{u}^{n}(0), v\right)_{\mathbb{L}^{2}}\right|^{2}\right] \leq\|v\|_{\mathbb{L}^{2}}^{2} \overline{\mathbb{E}}\left[\left\|\bar{u}^{n}(0)\right\|_{\mathbb{L}^{2}}^{2}\right] \leq c\|v\|_{\mathbb{H}_{0}^{1}}^{2} \overline{\mathbb{E}}\left[\sup _{0 \leq s \leq T}\left\|\bar{u}^{n}(s)\right\|_{\mathbb{L}^{2}}^{2}\right] \leq C,
$$

(5.31), (5.32) and Vitali Theorem gives

$$
\lim _{n \rightarrow \infty} \overline{\mathbb{E}}\left[\left|\left(\bar{u}^{n}(0)-u^{*}(0), v\right)_{\mathbb{L}^{2}}\right|\right]=0 .
$$

(iii) Since $\bar{U}^{n} \rightarrow U^{*}$ in $\mathbb{L}_{w}^{2}(\mathcal{O}), \bar{P}$-a.s and as $v \in \mathbb{H}_{0}^{1}(\mathcal{O}) \subset \mathbb{L}^{2}(\mathcal{O})$ we have

$$
\left(\bar{U}^{n}, v\right)_{\mathbb{L}^{2}} \rightarrow\left(U^{*}, v\right)_{\mathbb{L}^{2}}, \quad \bar{P}-\text { a.s. }
$$

Since $\overline{\mathbb{E}}\left[\left|\left(\bar{U}^{n}, v\right)_{\mathbb{L}^{2}}\right|^{2}\right] \leq\|v\|_{\mathbb{L}^{2}}^{2} \overline{\mathbb{E}}\left\|\bar{U}^{n}\right\|_{\mathbb{L}^{2}}^{2} \leq C$, by the Vitali theorem

$$
\lim _{n \rightarrow \infty} \overline{\mathbb{E}}\left[\left|\left(\bar{U}^{n}-U^{*}, v\right)_{\mathbb{L}^{2}}\right|\right]=0 .
$$

(iv) We see from (3.7) that $u \mapsto \int_{0}^{t}(A u(s), v)_{\mathbb{L}^{2}} d s$ from $L^{2}\left(0, T ; \mathbb{H}_{0}^{1}(\mathcal{O})\right)$ to $\mathbb{R}$ is linear and continuous. Since $\bar{u}^{n} \rightarrow u^{*}$ in $L_{w}^{2}\left(0, T ; \mathbb{H}_{0}^{1}(\mathcal{O})\right), \bar{P}$-a.s. we have,

$$
\lim _{n \rightarrow \infty} \int_{0}^{t}\left(A \bar{u}^{n}(s), v\right)_{\mathbb{L}^{2}} d s=\int_{0}^{t}\left(A u^{*}(s), v\right)_{\mathbb{L}^{2}} d s \quad \bar{P}-\text { a.s. }
$$

Hence by Hölder's inequality and (5.18), we achieve for all $t \in[0, T]$ and $n \in \mathbb{N}$,

$$
\overline{\mathbb{E}}\left[\left|\int_{0}^{t}\left(A \bar{u}^{n}(s), v\right)_{\mathbb{L}^{2}} d s\right|^{2}\right] \leq C_{1}\|v\|_{\mathbb{H}_{0}^{1}}^{2} \overline{\mathbb{E}}\left[\int_{0}^{t}\left\|\bar{u}^{n}(s)\right\|_{\mathbb{H}_{0}^{1}}^{2} d s\right] \leq c C_{2(2)}
$$

for some constant $c>0$. Therefore by (5.33), (5.34) and Vitali theorem, for all $t \in[0, T]$

$$
\lim _{n \rightarrow \infty} \overline{\mathbb{E}}\left[\left|\int_{0}^{t}\left(A \bar{u}^{n}(s)-A u^{*}(s), v\right)_{\mathbb{L}^{2}} d s\right|\right]=0 .
$$


Hence by the dominated convergence theorem

$$
\lim _{n \rightarrow \infty} \int_{0}^{T} \overline{\mathbb{E}}\left[\left|\int_{0}^{t}\left(A \bar{u}^{n}(s)-A u^{*}(s), v\right)_{\mathbb{L}^{2}} d s\right|\right]=0 .
$$

(v) First we note that $\bar{P}$-a.s. $\left\|w_{0}\right\|_{L^{4}\left(0, T ; \mathbb{L}^{4}\right)} \leq C$ and using (5.14) we have $\bar{P}$-a.s. $\left\|\bar{u}^{n}\right\|_{L^{2}\left(0, T ; \mathbb{H}_{0}^{1}\right)} \leq$ $C$ and $\left\|u^{*}\right\|_{L^{2}\left(0, T ; \mathbb{H}_{0}^{1}\right)} \leq C$ for some $C>0$. Exploiting (3.9), (3.12), Hölders inequality with $\frac{1}{2}+\frac{1}{4}+\frac{1}{4}=1$, and then using $\bar{u}^{n} \rightarrow u^{*}$ in $L^{2}\left(0, T ; \mathbb{L}^{2}(\mathcal{O})\right) \bar{P}$-a.s., we infer that $\bar{P}$-a.s.

$$
\begin{aligned}
& \left|\int_{0}^{t}\left(B\left(\bar{u}^{n}(s)\right)-B\left(u^{*}(s)\right), v\right)_{\mathbb{L}^{2}} d s\right| \\
& =\gamma\left|\int_{0}^{t}\left(\left|\bar{u}^{n}(s)+w_{0}(s)\right|\left(\bar{u}^{n}(s)+w_{0}(s)\right)-\left|u^{*}(s)+w_{0}(s)\right|\left(u^{*}(s)+w_{0}(s)\right), v\right)_{\mathbb{L}^{2}} d s\right| \\
& =\gamma \mid \int_{0}^{t}\left(\left|\bar{u}^{n}(s)+w_{0}(s)\right|\left(\bar{u}^{n}(s)+w_{0}(s)-\left(u^{*}(s)+w_{0}(s)\right)\right), v\right)_{\mathbb{L}^{2}} d s \\
& +\int_{0}^{t}\left(\left(\left|\bar{u}^{n}(s)+w_{0}(s)\right|-\left|u^{*}(s)+w_{0}(s)\right|\right)\left(u^{*}(s)+w_{0}(s)\right), v\right)_{\mathbb{L}^{2}} d s \mid \\
& \leq \gamma \int_{0}^{t}\left|\left(\left|\bar{u}^{n}(s)+w_{0}(s)\right|\left(\bar{u}^{n}(s)-u^{*}(s)\right), v\right)_{\mathbb{L}^{2}} d s\right| \\
& +\gamma \int_{0}^{t}\left|\left(\left(\left|\bar{u}^{n}(s)+w_{0}(s)\right|-\left|u^{*}(s)+w_{0}(s)\right|\right)\left(u^{*}(s)+w_{0}(s)\right), v\right)_{\mathbb{L}^{2}} d s\right| \\
& \leq \gamma \int_{0}^{t}\left\|\bar{u}^{n}(s)+w_{0}(s)\right\|_{\mathbb{L}^{4}}\left\|\bar{u}^{n}(s)-u^{*}(s)\right\|_{\mathbb{L}^{2}}\|v\|_{\mathbb{L}^{4}} d s \\
& +\gamma \int_{0}^{t}\left\|\left|\bar{u}^{n}(s)+w_{0}(s)\right|-\left|u^{*}(s)+w_{0}(s)\right|\right\|_{\mathbb{L}^{2}}\left\|u^{*}(s)+w_{0}(s)\right\|_{\mathbb{L}^{4}}\|v\|_{\mathbb{L}^{4}} d s \\
& \leq 2 \gamma\left(\int_{0}^{t}\left\|\bar{u}^{n}(s)-u^{*}(s)\right\|_{\mathbb{L}^{2}}\|v\|_{\mathbb{L}^{4}}\left(\left\|\bar{u}^{n}(s)+w_{0}(s)\right\|_{\mathbb{L}^{4}}+\left\|u^{*}(s)+w_{0}(s)\right\|_{\mathbb{L}^{4}}\right) d s\right) \\
& \leq 2 \gamma\|v\|_{\mathbb{L}^{4}}\left[\left(\int_{0}^{T}\left\|\bar{u}^{n}(s)-u^{*}(s)\right\|_{\mathbb{L}^{2}}\left\|\bar{u}^{n}(s)+w_{0}(s)\right\|_{\mathbb{L}^{4}} d s\right)\right. \\
& \left.+\left(\int_{0}^{T}\left\|\bar{u}^{n}(s)-u^{*}(s)\right\|_{\mathbb{L}^{2}}\left\|u^{*}(s)+w_{0}(s)\right\|_{\mathbb{L}^{4}} d s\right)\right] \\
& \leq c\|v\|_{\mathbb{H}_{0}^{1}}\left[\left\|\bar{u}^{n}+w_{0}\right\|_{L^{2}\left(0, T ; \mathbb{L}^{4}\right)}+\left\|u^{*}+w_{0}\right\|_{L^{2}\left(0, T ; \mathbb{L}^{4}\right)}\right] \\
& \times\left\|\bar{u}^{n}-u^{*}\right\|_{L^{2}\left(0, T ; \mathbb{L}^{2}\right)} \\
& \leq c\|v\|_{\mathbb{H}_{0}^{1}}\left[\left\|\bar{u}^{n}\right\|_{L^{2}\left(0, T ; \mathbb{L}^{4}\right)}+2\left\|w_{0}\right\|_{L^{2}\left(0, T ; \mathbb{L}^{4}\right)}+\left\|u^{*}\right\|_{L^{2}\left(0, T ; \mathbb{L}^{4}\right)}\right]\left\|\bar{u}^{n}-u^{*}\right\|_{L^{2}\left(0, T ; \mathbb{L}^{2}\right)} \\
& \leq c\|v\|_{\mathbb{H}_{0}^{1}}\left[\left\|\bar{u}^{n}\right\|_{L^{2}\left(0, T ; \mathbb{H}_{0}^{1}\right)}+2\left\|w_{0}\right\|_{L^{4}\left(0, T ; \mathbb{L}^{4}\right)}+\left\|u^{*}\right\|_{L^{2}\left(0, T ; \mathbb{H}_{0}^{1}\right)}\right]\left\|\bar{u}^{n}-u^{*}\right\|_{L^{2}\left(0, T ; \mathbb{L}^{2}\right)} \\
& \leq C\left\|\bar{u}^{n}-u^{*}\right\|_{L^{2}\left(0, T ; \mathbb{L}^{2}\right)} \rightarrow 0 \quad \text { as } \quad n \rightarrow \infty \text {. }
\end{aligned}
$$

Employing (5.17), (5.18), (3.11), Lemma 3.1. Proposition 4.4, we observe that for every $t \in[0, T]$ and $1 \leq r<2$, and for every $n \in \mathbb{N}$,

$$
\begin{aligned}
& \overline{\mathbb{E}}\left[\left|\int_{0}^{t}\left(B\left(\bar{u}^{n}(s)\right), v\right)_{\mathbb{L}^{2}} d s\right|^{r}\right] \leq c\|v\|_{\mathbb{L}^{2}}^{r} T^{r-1} \overline{\mathbb{E}}\left[\int_{0}^{t}\left\|B\left(\bar{u}^{n}(s)\right)\right\|_{\mathbb{L}^{2}}^{r} d s\right] \\
& \leq c C_{2}\|v\|_{\mathbb{L}^{2}}^{r} T^{r-1} \overline{\mathbb{E}}\left[\int_{0}^{t}\left\|\bar{u}^{n}(s)+w^{0}(s)\right\|_{\mathbb{L}^{4}}^{2 r} d s\right] \\
& \leq C\|v\|_{\mathbb{L}^{2}}^{r} T^{r-1}\left\{\overline{\mathbb{E}}\left[\int_{0}^{T}\left(\left\|\bar{u}^{n}(s)\right\|_{\mathbb{L}^{4}}^{2 r}+\left\|w^{0}(s)\right\|_{\mathbb{L}^{4}}^{2 r}\right) d s\right]\right\} \leq C,
\end{aligned}
$$


since,

$$
\begin{aligned}
& \overline{\mathbb{E}}\left[\int_{0}^{T}\left\|\bar{u}^{n}(s)\right\|_{\mathbb{L}^{4}}^{2 r} d s\right] \leq 2^{\frac{r}{2}} \overline{\mathbb{E}}\left[\int_{0}^{T}\left\|\bar{u}^{n}(s)\right\|_{\mathbb{L}^{2}}^{r}\left\|\bar{u}^{n}(s)\right\|_{\mathbb{H}_{0}^{1}}^{r} d s\right] \\
& \leq C_{r}\left(\overline{\mathbb{E}}\left[\int_{0}^{T}\left\|\bar{u}^{n}(s)\right\|_{\mathbb{H}_{0}^{1}}^{2} d s\right]+\overline{\mathbb{E}}\left[T \sup _{0 \leq s \leq T}\left\|\bar{u}^{n}(s)\right\|_{\mathbb{L}^{2}}^{\frac{2 r}{2-r}}\right]\right) \leq C,
\end{aligned}
$$

provided $\frac{2 r}{2-r} \geq 2$. Hence (5.35), (5.36) and by Vitali theorem, for all $t \in[0, T]$, we have

$$
\lim _{n \rightarrow \infty} \overline{\mathbb{E}}\left[\left|\int_{0}^{t}\left(B\left(\bar{u}^{n}(s)\right)-B\left(u^{*}(s)\right), v\right)_{\mathbb{L}^{2}} d s\right|\right]=0 .
$$

Hence by the dominated convergence theorem

$$
\lim _{n \rightarrow \infty} \int_{0}^{T} \overline{\mathbb{E}}\left[\left|\int_{0}^{t}\left(B\left(\bar{u}^{n}(s)\right)-B\left(u^{*}(s)\right), v\right)_{\mathbb{L}^{2}} d s\right|\right] d t=0 .
$$

(vi) Since $\bar{z}^{n} \rightarrow z^{*}$ in $L^{2}\left(0, T ; L^{2}(\mathcal{O})\right) \bar{P}$-a.s., so for any $\tilde{\phi} \in L^{2}\left(0, T ; \mathbb{H}_{0}^{1}(\mathcal{O})\right)$, using Hölder inequality we have $\bar{P}-$ a.s.,

$$
\begin{aligned}
& \left|\int_{0}^{T}\left\langle g \nabla\left(\bar{z}^{n}(s)-z^{*}(s)\right), \tilde{\phi}(s)\right\rangle d s\right|=\left|-\int_{0}^{T}\left(g\left(\bar{z}^{n}(s)-z^{*}(s)\right), \nabla \cdot \tilde{\phi}(s)\right)_{L^{2}} d s\right| \\
& \leq \int_{0}^{T}\left|\left(g\left(\bar{z}^{n}(s)-z^{*}(s)\right), \nabla \cdot \tilde{\phi}(s)\right)_{L^{2}}\right| d s \\
& \leq g\left(\int_{0}^{T}\left\|\bar{z}^{n}(s)-z^{*}(s)\right\|_{L^{2}} d s\right)^{1 / 2}\left(\int_{0}^{T}\|\nabla \cdot \tilde{\phi}(s)\|_{L^{2}}^{2} d s\right)^{1 / 2} \rightarrow 0 \quad \text { as } n \rightarrow \infty .
\end{aligned}
$$

In particular, we choose $\tilde{\phi}(s)=\chi_{(0, t)}(s) v$, where $v$ is a fixed element of $\mathbb{H}_{0}^{1}(\mathcal{O})$. Hence, from (5.37), we have for all $t \in[0, T] \bar{P}$-a.s.

$$
\lim _{n \rightarrow \infty} \int_{0}^{t}\left\langle g \nabla\left(\bar{z}^{n}(s)-z^{*}(s)\right), v\right\rangle d s=0
$$

Now by (5.19)

$$
\begin{aligned}
& \overline{\mathbb{E}}\left[\mid \int_{0}^{t}\left\langle\left. g \nabla\left(\bar{z}^{n}(s), v\right\rangle d s\right|^{2}\right] \leq g \overline{\mathbb{E}}\left[\int_{0}^{t}\left|\left(\bar{z}^{n}(s), \nabla \cdot v\right)_{L^{2}}\right|^{2} d s\right]\right. \\
& \leq g\|v\|_{\mathbb{H}_{0}^{1}}^{2} \overline{\mathbb{E}}\left[\int_{0}^{t}\left\|\bar{z}^{n}(s)\right\|_{L^{2}}^{2} d s\right] \leq g T\|v\|_{\mathbb{H}_{0}^{1}}^{2} \overline{\mathbb{E}}\left[\sup _{0 \leq s \leq T}\left\|\bar{z}^{n}(s)\right\|_{L^{2}}^{2}\right] \\
& \leq C .
\end{aligned}
$$

Hence from (5.38), (5.39) and applying Vitali's theorem we obtain, for all $t \in[0, T]$,

$$
\lim _{n \rightarrow \infty} \overline{\mathbb{E}}\left[\left|\int_{0}^{t}\left\langle g \nabla\left(\bar{z}^{n}(s)-z^{*}(s)\right), v\right\rangle d s\right|\right]=0 .
$$

Again using (5.19) and employing Cauchy-Schwartz inequality twice, we achieve

$$
\begin{aligned}
& \left|\overline{\mathbb{E}}\left[\int_{0}^{t}\left\langle g \nabla \bar{z}^{n}(s), v\right\rangle d s\right]\right| \leq g \overline{\mathbb{E}}\left[\int_{0}^{t}\left|\left(\bar{z}^{n}(s), \nabla \cdot v\right)_{L^{2}}\right| d s\right] \\
& \leq g\|v\|_{\mathbb{H}_{0}^{1}} \overline{\mathbb{E}}\left[\int_{0}^{t}\left\|\bar{z}^{n}(s)\right\|_{L^{2}} d s\right] \leq g\|v\|_{\mathbb{H}_{0}^{1}} T^{\frac{1}{2}}\left[\overline{\mathbb{E}} \int_{0}^{t}\left\|\bar{z}^{n}(s)\right\|_{L^{2}}^{2} d s\right]^{\frac{1}{2}} \\
& \leq g\|v\|_{\mathbb{H}_{0}^{1}} T^{\frac{3}{2}} \overline{\mathbb{E}}\left[\sup _{0 \leq s \leq T}\left\|\bar{z}^{n}(s)\right\|_{L^{2}}^{2}\right]^{\frac{1}{2}} \leq C .
\end{aligned}
$$

Hence by (5.40), (5.41) and by the Dominated Convergence theorem we have

$$
\lim _{n \rightarrow \infty} \int_{0}^{T} \overline{\mathbb{E}}\left[\left|\int_{0}^{t}\left\langle g \nabla\left(\bar{z}^{n}(s)-z^{*}(s)\right), v\right\rangle d s\right|\right] d t=0 .
$$


(vii) Using the Assumption 3.12 H.3, since $\bar{u}^{n} \rightarrow u^{*}$ in $L^{2}\left(0, T ; \mathbb{L}^{2}(\mathcal{O})\right) \bar{P}$-a.s., we have $\bar{P}$-a.s.

$$
\begin{aligned}
& \lim _{n \rightarrow \infty} \int_{0}^{t}\left\|\left(\sigma\left(s, \bar{u}^{n}(s)\right)-\sigma\left(s, u^{*}(s)\right), v\right)_{\mathbb{L}^{2}}\right\|_{L_{Q}\left(\mathbb{L}^{2} ; \mathbb{R}\right)}^{2} d s \\
& \leq \lim _{n \rightarrow \infty}\|v\|_{\mathbb{L}^{2}}^{2} \int_{0}^{t}\left\|\sigma\left(s, \bar{u}^{n}(s)\right)-\sigma\left(s, u^{*}(s)\right)\right\|_{L_{Q}\left(\mathbb{L}^{2} ; \mathbb{R}\right)}^{2} d s \\
& \leq L\|v\|_{\mathbb{L}^{2}}^{2} \lim _{n \rightarrow \infty} \int_{0}^{T}\left\|\bar{u}^{n}(s)-u^{*}(s)\right\|_{\mathbb{L}^{2}}^{2} d s=0 .
\end{aligned}
$$

Using Assumption 3.12 H.2, (5.22) (in Proposition 5.14) and (5.17) we observe that for every $t \in[0, T]$ and $r>1$ and for every $n \in \mathbb{N}$,

$$
\begin{aligned}
& \overline{\mathbb{E}}\left[\left|\int_{0}^{t}\left\|\left(\sigma\left(s, \bar{u}^{n}(s)\right)-\sigma\left(s, u^{*}(s)\right), v\right)_{\mathbb{L}^{2}}\right\|_{L_{Q}\left(\mathbb{L}^{2} ; \mathbb{R}\right)}^{2} d s\right|^{r}\right] \\
& \leq\|v\|_{\mathbb{L}^{2}}^{2 r} T^{r-1} 2^{2 r-1} \overline{\mathbb{E}}\left[\int_{0}^{t}\left(\left\|\sigma\left(s, \bar{u}^{n}(s)\right)\right\|_{L_{Q}\left(\mathbb{L}^{2} ; \mathbb{L}^{2}\right)}^{2 r}+\left\|\sigma\left(s, u^{*}(s)\right)\right\|_{L_{Q}\left(\mathbb{L}^{2} ; \mathbb{L}^{2}\right)}^{2 r}\right) d s\right] \\
& \leq K\|v\|_{\mathbb{L}^{2}}^{2 r} T^{r-1} 2^{2 r-1} \overline{\mathbb{E}}\left[\int_{0}^{t}\left(2+\left\|\bar{u}^{n}(s)\right\|_{\mathbb{L}^{2}}^{2 r}+\left\|u^{*}(s)\right\|_{\mathbb{L}^{2}}^{2 r}\right) d s\right] \\
& \leq c_{r} \overline{\mathbb{E}}\left[\left(2+\sup _{0 \leq s \leq T}\left\|\bar{u}^{n}(s)\right\|_{\mathbb{L}^{2}}^{2 r}+\sup _{0 \leq s \leq T}\left\|u^{*}(s)\right\|_{\mathbb{L}^{2}}^{2 r}\right)\right] \leq \tilde{c}_{r},
\end{aligned}
$$

for some constant $\tilde{c}_{r}>0$ (depending upon $r$ ). Thus by employing (5.42) and (5.43) and Vitali's theorem we obtain

$$
\lim _{n \rightarrow \infty} \overline{\mathbb{E}}\left[\int_{0}^{t}\left\|\left(\sigma\left(s, \bar{u}^{n}(s)\right)-\sigma\left(s, u^{*}(s)\right), v\right)_{\mathbb{L}^{2}}\right\|_{L_{Q}\left(\mathbb{L}^{2} ; \mathbb{R}\right)}^{2} d s\right]=0 \quad \forall v \in \mathbb{H}_{0}^{1}(\mathcal{O}) .
$$

For every $v \in \mathbb{H}_{0}^{1}(\mathcal{O})$ and every $s \in[0, T]$ we have

$$
\begin{aligned}
& \left(\sigma^{n}\left(s, \bar{u}^{n}(s)\right)-\sigma\left(s, u^{*}(s)\right), v\right)_{\mathbb{L}^{2}}=\left(\sigma\left(s, \bar{u}^{n}(s)\right), P_{n} v\right)_{\mathbb{L}^{2}}-\left(\sigma\left(s, u^{*}(s)\right), v\right)_{\mathbb{L}^{2}} \\
& =\left(\sigma\left(s, \bar{u}^{n}(s)\right), P_{n} v-v\right)_{\mathbb{L}^{2}}+\left(\sigma\left(s, \bar{u}^{n}(s)\right)-\sigma\left(s, u^{*}(s)\right), v\right)_{\mathbb{L}^{2}} \\
& \leq\left\|\sigma\left(s, \bar{u}^{n}(s)\right)\right\|_{L_{Q}\left(\mathbb{L}^{2} ; \mathbb{L}^{2}\right)}\left\|P_{n} v-v\right\|_{\mathbb{L}^{2}}+\left(\sigma\left(s, \bar{u}^{n}(s)\right)-\sigma\left(s, u^{*}(s)\right), v\right)_{\mathbb{L}^{2}} \\
& \leq c\left\|\sigma\left(s, \bar{u}^{n}(s)\right)\right\|_{L_{Q}\left(\mathbb{L}^{2} ; \mathbb{L}^{2}\right)}\left\|P_{n} v-v\right\|_{\mathbb{H}_{0}^{1}}+\left(\sigma\left(s, \bar{u}^{n}(s)\right)-\sigma\left(s, u^{*}(s)\right), v\right)_{\mathbb{L}^{2}} .
\end{aligned}
$$

Then by Assumption 3.12 H.2 and by (5.17) we obtain

$$
\begin{aligned}
& \overline{\mathbb{E}}\left[\int_{0}^{t}\left\|\left(\sigma^{n}\left(s, \bar{u}^{n}(s)\right)-\sigma\left(s, u^{*}(s)\right), v\right)_{\mathbb{L}^{2}}\right\|_{L_{Q}\left(\mathbb{L}^{2} ; \mathbb{R}\right)}^{2} d s\right] \\
& \leq 2 c K_{2}\left\|P_{n} v-v\right\|_{\mathbb{H}_{0}^{1}}^{2} \overline{\mathbb{E}}\left[\int_{0}^{T}\left(1+\left\|\bar{u}^{n}(s)\right\|_{\mathbb{L}^{2}}^{2}\right) d s\right] \\
& \quad+\overline{\mathbb{E}}\left[\int_{0}^{t} \|\left(\sigma\left(s, \bar{u}^{n}(s)\right)-\left(\sigma\left(s, u^{*}(s)\right), v\right)_{\mathbb{L}^{2}} \|_{L_{Q}\left(\mathbb{L}^{2} ; \mathbb{R}\right)}^{2} d s\right]\right. \\
& \leq 2 \tilde{c}\left\|P_{n} v-v\right\|_{\mathbb{H}_{0}^{1}}^{2}+\overline{\mathbb{E}}\left[\int_{0}^{t} \|\left(\sigma\left(s, \bar{u}^{n}(s)\right)-\left(\sigma\left(s, u^{*}(s)\right), v\right)_{\mathbb{L}^{2}} \|_{L_{Q}\left(\mathbb{L}^{2} ; \mathbb{R}\right)}^{2} d s\right] .\right.
\end{aligned}
$$

Thus by Lemma 4.1 and by (5.44) we conclude that

$\lim _{n \rightarrow \infty} \overline{\mathbb{E}}\left[\int_{0}^{t}\left\|\left(\sigma^{n}\left(s, \bar{u}^{n}(s)\right)-\sigma\left(s, u^{*}(s)\right), v\right)_{\mathbb{L}^{2}}\right\|_{L_{Q}\left(\mathbb{L}^{2} ; \mathbb{R}\right)}^{2} d s\right]=0 \quad \forall v \in \mathbb{H}_{0}^{1}(\mathcal{O})$.

Using $\bar{W}^{n}=W^{*}$, Itô isometry, (5.45) and $\forall v \in \mathbb{H}_{0}^{1}(\mathcal{O})$ we have

$$
\begin{aligned}
& \lim _{n \rightarrow \infty} \overline{\mathbb{E}}\left[\left\|\left(\int_{0}^{t}\left(\sigma^{n}\left(s, \bar{u}^{n}(s)\right) d \bar{W}^{n}(s)-\sigma\left(s, u^{*}(s)\right)\right) d W^{*}(s), v\right)_{\mathbb{L}^{2}}\right\|_{L_{Q}\left(\mathbb{L}^{2} ; \mathbb{R}\right)}^{2}\right] \\
= & \lim _{n \rightarrow \infty} \overline{\mathbb{E}}\left[\left\|\left(\int_{0}^{t}\left(\sigma^{n}\left(s, \bar{u}^{n}(s)\right)-\sigma\left(s, u^{*}(s)\right)\right) d W^{*}(s), v\right)_{\mathbb{L}^{2}}\right\|_{L_{Q}\left(\mathbb{L}^{2} ; \mathbb{R}\right)}^{2}\right] \\
= & \lim _{n \rightarrow \infty} \overline{\mathbb{E}}\left[\int_{0}^{t}\left\|\left(\sigma^{n}\left(s, \bar{u}^{n}(s)\right)-\sigma\left(s, u^{*}(s)\right), v\right)_{\mathbb{L}^{2}}\right\|_{L_{Q}\left(\mathbb{L}^{2} ; \mathbb{R}\right)}^{2} d s\right]=0
\end{aligned}
$$


Again by the Itô isometry, (4.18), Assumption 3.12 and (5.17) we have for all $t \in[0, T]$ and all $n \in \mathbb{N}$,

$$
\begin{aligned}
& \overline{\mathbb{E}}\left[\int_{0}^{t}\left\|\left(\left[\sigma^{n}\left(s, \bar{u}^{n}(s)\right)-\sigma\left(s, u^{*}(s)\right)\right] d W^{*}(s), v\right)_{\mathbb{L}^{2}}\right\|_{L_{Q}\left(\mathbb{L}^{2} ; \mathbb{R}\right)}^{2} d s\right] \\
& =\overline{\mathbb{E}}\left[\int_{0}^{t}\left\|\left(\sigma^{n}\left(s, \bar{u}^{n}(s)\right)-\sigma\left(s, u^{*}(s)\right), v\right)_{\mathbb{L}^{2}}\right\|_{L_{Q}\left(\mathbb{L}^{2} ; \mathbb{R}\right)}^{2} d s\right] \\
& \leq 2 c K_{2} T^{2}\|v\|_{\mathbb{H}_{0}^{1}}^{2} \overline{\mathbb{E}}\left[\left(2+\sup _{0 \leq s \leq T}\left\|\bar{u}^{n}(s)\right\|_{\mathbb{L}^{2}}^{2}+\sup _{0 \leq s \leq T}\left\|u^{*}(s)\right\|_{\mathbb{L}^{2}}^{2}\right)\right] \leq \tilde{C}_{2} .
\end{aligned}
$$

Hence by (5.46) and (5.47) and the Dominated Convergence Theorem, we have the assertion (vii).

(viii) Using Assumption 3.12 H.3 and that $\bar{u}^{n} \rightarrow u^{*}$ in $L^{2}\left(0, T ; \mathbb{L}^{2}(\mathcal{O})\right) \bar{P}$-a.s., we have $\bar{P}$-a.s.

$$
\begin{aligned}
& \int_{0}^{t} \int_{Z}\left|\left(H\left(\bar{u}^{n}(s), z\right)-H\left(u^{*}(s), z\right), v\right)_{\mathbb{L}^{2}}\right|^{2} \lambda(d z) d s \\
& \leq\|v\|_{\mathbb{L}^{2}}^{2} \int_{0}^{t} \int_{Z} \|\left(H\left(\bar{u}^{n}(s), z\right)-H\left(u^{*}(s), z\right) \|_{\mathbb{L}^{2}}^{2} \lambda(d z) d s\right. \\
& \leq L\|v\|_{\mathbb{L}^{2}}^{2} \int_{0}^{T}\left\|\bar{u}^{n}(s)-u^{*}(s)\right\|_{\mathbb{L}^{2}}^{2} d s \rightarrow 0 \text { as } n \rightarrow \infty .
\end{aligned}
$$

Furthermore, using Assumption 3.12 H.2, (5.22) (in Proposition 5.14) and (5.17), for every $t \in[0, T], r>1$ and $n \in \mathbb{N}$, we have the following inequality

$$
\begin{aligned}
& \overline{\mathbb{E}}\left[\left.\left.\left|\int_{0}^{t} \int_{Z}\right|\left(H\left(\bar{u}^{n}(s), z\right)-H\left(u^{*}(s), z\right), v\right)_{\mathbb{L}^{2}}\right|^{2} \lambda(d z) d s\right|^{r}\right] \\
& \leq 2^{r}\|v\|_{\mathbb{L}^{2}}^{2 r} \overline{\mathbb{E}}\left[\left|\int_{0}^{t} \int_{Z}\left(\left\|H\left(\bar{u}^{n}(s), z\right)\right\|_{\mathbb{L}^{2}}^{2}+\left\|H\left(u^{*}(s), z\right)\right\|_{\mathbb{L}^{2}}^{2}\right) \lambda(d z) d s\right|^{r}\right] \\
& \leq 2^{r} K^{2 r}\|v\|_{\mathbb{L}^{2}}^{2 r} \overline{\mathbb{E}}\left[\left|\int_{0}^{t}\left(2+\left\|\bar{u}^{n}(s)\right\|_{\mathbb{L}^{2}}^{2}+\left\|u^{*}(s)\right\|_{\mathbb{L}^{2}}^{2}\right) d s\right|^{r}\right] \\
& \left.\leq 2^{r} K^{2 r}\|v\|_{\mathbb{L}^{2}}^{2 r} T^{r}\left(1+\overline{\mathbb{E}}\left[\sup _{0 \leq s \leq T}\left\|\bar{u}^{n}(s)\right\|_{\mathbb{L}^{2}}^{2 r}\right]+\overline{\mathbb{E}}\left[\sup _{0 \leq s \leq T}\left\|u^{*}(s)\right\|_{\mathbb{L}^{2}}^{2 r}\right)\right]\right) \\
& \leq \tilde{c}_{r}
\end{aligned}
$$

for some constant $\tilde{c}_{r}>0$ (depending upon $r$ ). Thus by (5.48) and (5.49) and by Vitali's theorem for every $t \in[0, T], \forall v \in \mathbb{H}_{0}^{1}(\mathcal{O})$,

$$
\lim _{n \rightarrow \infty} \overline{\mathbb{E}}\left[\int_{0}^{t} \int_{Z}\left|\left(H\left(\bar{u}^{n}(s), z\right)-H\left(u^{*}(s), z\right), v\right)_{\mathbb{L}^{2}}\right|^{2} \lambda(d z) d s\right]=0 .
$$

Since the restriction of $P_{n}$ to $\mathbb{L}^{2}(\mathcal{O})$, is the $(\cdot, \cdot)_{\mathbb{L}^{2}}$-orthogonal projection onto $\mathbb{L}_{n}^{2}(\mathcal{O})$, we conclude $\forall v \in \mathbb{L}^{2}(\mathcal{O})$,

$$
\lim _{n \rightarrow \infty} \overline{\mathbb{E}}\left[\int_{0}^{t} \int_{Z}\left|\left(H^{n}\left(\bar{u}^{n}(s), z\right)-H\left(u^{*}(s), z\right), v\right)_{\mathbb{L}^{2}}\right|^{2} \lambda(d z) d s\right]=0 .
$$

Since $\mathbb{H}_{0}^{1}(\mathcal{O}) \subset \mathbb{L}^{2}(\mathcal{O})$, (5.51) holds for all $v \in \mathbb{H}_{0}^{1}(\mathcal{O})$.

Moreover, Assumption 3.12 and (5.17) yield the following inequality

$$
\overline{\mathbb{E}}\left[\int_{0}^{t} \int_{Z}\left|\left(H^{n}\left(\bar{u}^{n}(s), z\right)-H\left(u^{*}(s), z\right), v\right)_{\mathbb{L}^{2}}\right|^{2} \lambda(d z) d s\right] \leq \tilde{C}_{2} .
$$

Now (5.51), (5.52) and the Dominated Convergence Theorem assures assertion (viii).

(ix) Employing $\bar{N}^{n}=N^{*}$, Itô-Lévy isometry and (5.51), we have $\forall v \in \mathbb{H}_{0}^{1}(\mathcal{O})$

$$
\lim _{n \rightarrow \infty} \overline{\mathbb{E}}\left[\left|\int_{0}^{t} \int_{Z}\left(H^{n}\left(\bar{u}^{n}(s-), z\right)-H\left(u^{*}(s-), z\right), v\right)_{\mathbb{L}^{2}} \tilde{\bar{N}}^{n}(d s, d z)\right|^{2}\right]
$$




$$
\begin{aligned}
& =\lim _{n \rightarrow \infty} \overline{\mathbb{E}}\left[\left|\int_{0}^{t} \int_{Z}\left(H^{n}\left(\bar{u}^{n}(s-), z\right)-H\left(u^{*}(s-), z\right), v\right)_{\mathbb{L}^{2}} \tilde{N}^{*}(d s, d z)\right|^{2}\right] \\
& =\lim _{n \rightarrow \infty} \overline{\mathbb{E}}\left[\int_{0}^{t} \int_{Z}\left|\left(H^{n}\left(\bar{u}^{n}(s), z\right)-H\left(u^{*}(s), z\right), v\right)_{\mathbb{L}^{2}}\right|^{2} \lambda(d z) d s\right]=0 .
\end{aligned}
$$

Moreover from (5.52), we have $\forall v \in \mathbb{H}_{0}^{1}(\mathcal{O})$

$$
\overline{\mathbb{E}}\left[\left|\int_{0}^{t} \int_{Z}\left(H^{n}\left(\bar{u}^{n}(s-), z\right)-H\left(u^{*}(s-), z\right), v\right)_{\mathbb{L}^{2}} \tilde{N}^{*}(d s, d z)\right|^{2}\right] \leq \tilde{C}_{2} .
$$

Hence by (5.53), (5.54) and the Dominated convergence theorem we obtain (ix).

(x) Since $\bar{z}^{n} \rightarrow z^{*}$ in $C\left([0, T] ; H^{-1}(\mathcal{O})\right) \bar{P}$-a.s., we see that $\bar{P}$-a.s. and for all $t \in[0, T]$

$$
\begin{aligned}
& \left|\left\langle\bar{z}^{n}(t)-z^{*}(t), w\right\rangle\right| \leq\left\|\bar{z}^{n}(t)-z^{*}(t)\right\|_{H^{-1}}\|w\|_{H_{0}^{1}} \\
& \leq\|w\|_{H_{0}^{1}} \sup _{t \in[0, T]}\left\|\bar{z}^{n}(t)-z^{*}(t)\right\|_{H^{-1}} \rightarrow 0 \quad \text { as } n \rightarrow \infty .
\end{aligned}
$$

Also from (5.19) we get

$$
\begin{aligned}
\overline{\mathbb{E}}\left[\int_{0}^{T}\left|\left\langle\bar{z}^{n}(t), w\right\rangle\right|^{2} d t\right] & \leq\|w\|_{H_{0}^{1}}^{2} \overline{\mathbb{E}}\left[\int_{0}^{T}\left\|\bar{z}^{n}(t)\right\|_{H^{-1}}^{2} d t\right] \\
& \leq c\|w\|_{H_{0}^{1}}^{2} T \overline{\mathbb{E}}\left[\sup _{0 \leq t \leq T}\left\|\bar{z}^{n}(t)\right\|_{L^{2}}^{2}\right] \leq C .
\end{aligned}
$$

By (5.55), (5.56) and then by the Vitali theorem we have (x).

(xi) Since $\bar{z}^{n} \rightarrow z^{*}$ in $C\left([0, T] ; H^{-1}(\mathcal{O})\right) \bar{P}$-a.s., using similar arguments as in (5.55) in particular for $t=0$ we have $\bar{P}$-a.s.

$$
\left\langle\bar{z}_{0}^{n}, w\right\rangle \rightarrow\left\langle z_{0}^{*}, w\right\rangle
$$

Hence by (5.19) we achieve

$$
\overline{\mathbb{E}}\left[\left|\left\langle\bar{z}_{0}^{n}, w\right\rangle\right|^{2}\right] \leq c\|w\|_{H_{0}^{1}}^{2} \overline{\mathbb{E}}\left[\left\|\bar{z}_{0}^{n}\right\|_{L^{2}}^{2}\right] \leq c\|w\|_{H_{0}^{1}}^{2} \overline{\mathbb{E}}\left[\left\|\bar{z}_{0}\right\|_{L^{2}}^{2}\right] \leq C .
$$

Then by (5.57) and (5.58) and using Vitali theorem

$$
\lim _{n \rightarrow \infty} \overline{\mathbb{E}}\left[\left|\left(\bar{z}_{0}^{n}-z_{0}^{*}, w\right)_{L^{2}}\right|\right]=0 .
$$

(xii) Since $\bar{u}^{n} \rightarrow u^{*}$ in $L_{w}^{2}\left(0, T ; \mathbb{H}_{0}^{1}(\mathcal{O})\right) \bar{P}$-a.s., so for any $\tilde{\phi} \in L^{2}\left(0, T ; L^{2}(\mathcal{O})\right)$ we have $\bar{P}$-a.s.,

$$
\lim _{n \rightarrow \infty} \int_{0}^{T}\left(\operatorname{Div}\left(h \bar{u}^{n}(s)\right), \tilde{\phi}(s)\right)_{L^{2}} d s=\int_{0}^{T}\left(\operatorname{Div}\left(h u^{*}(s)\right), \tilde{\phi}(s)\right)_{L^{2}} d s .
$$

Let $t \in[0, T]$ be fixed. Let us choose $\tilde{\phi}(s)=\chi_{(0, t)}(s) w$ in (5.59). Also we note that $\tilde{\phi} \in L^{2}\left(0, T ; L^{2}(\mathcal{O})\right)$. Hence,

$$
\lim _{n \rightarrow \infty} \int_{0}^{t}\left(\operatorname{Div}\left(h \bar{u}^{n}(s)\right), w\right)_{L^{2}} d s=\int_{0}^{t}\left(\operatorname{Div}\left(h u^{*}(s)\right), w\right)_{L^{2}} d s .
$$

By (5.18), Minkowskii and Hölder inequalities, and by (2.5) we have

$$
\begin{aligned}
& \overline{\mathbb{E}}\left[\left|\int_{0}^{t}\left(\operatorname{Div}\left(h \bar{u}^{n}(s)\right), w\right)_{L^{2}} d s\right|^{2}\right] \leq T\|w\|_{L^{2}}^{2} \overline{\mathbb{E}}\left[\int_{0}^{t}\left\|\operatorname{Div}\left(h \bar{u}^{n}(s)\right)\right\|_{L^{2}}^{2} d s\right] \\
& \quad \leq c T\|w\|_{L^{2}}^{2} \overline{\mathbb{E}}\left[\int_{0}^{t}\left(\left\|h \operatorname{Div} \bar{u}^{n}(s)\right\|\left\|_{L^{2}}^{2}+\right\| \nabla h \cdot \bar{u}^{n}(s) \|_{L^{2}}^{2}\right) d s\right] \\
& \quad \leq c T\|w\|_{H_{0}^{1}}^{2} \overline{\mathbb{E}}\left[\int_{0}^{t}\left(\|h\|_{L^{\infty}}^{2}\left\|\nabla \bar{u}^{n}(s)\right\|_{\mathbb{L}^{2}}^{2}+\|\nabla h\|_{\mathbb{L}^{\infty}}^{2}\left\|\bar{u}^{n}(s)\right\|_{\mathbb{L}^{2}}^{2}\right) d s\right] \\
& \quad \leq c T\|w\|_{H_{0}^{1}}^{2}\left(\mu^{2} \overline{\mathbb{E}} \int_{0}^{t}\left\|\bar{u}^{n}(s)\right\|_{\mathbb{H}_{0}^{1}}^{2} d s+M^{2} T \overline{\mathbb{E}} \sup _{0 \leq t \leq T}\left\|\bar{u}^{n}(s)\right\|_{\mathbb{L}^{2}}^{2}\right) \leq C .
\end{aligned}
$$


Therefore by (5.60) and (5.61) and by Vitali theorem, for all $t \in[0, T]$,

$$
\lim _{n \rightarrow \infty} \overline{\mathbb{E}}\left[\left|\int_{0}^{t}\left(\left(\operatorname{Div}\left(h \bar{u}^{n}(s)\right), w\right)_{L^{2}}-\left(\operatorname{Div}\left(h u^{*}(s)\right), w\right)_{L^{2}}\right) d s\right|\right]=0 .
$$

Also repeating the same arguments as for (5.61) we achieve,

$$
\int_{0}^{T}\left|\overline{\mathbb{E}}\left[\int_{0}^{t}\left(\operatorname{Div}\left(h \bar{u}^{n}(s)\right), w\right)_{L^{2}} d s\right]\right|^{2} d t \leq C T .
$$

Hence by (5.62) and (5.63) and by the virtue of Vitali theorem, we have

$$
\lim _{n \rightarrow \infty} \int_{0}^{T} \overline{\mathbb{E}}\left[\left|\int_{0}^{t}\left(\left(\operatorname{Div}\left(h \bar{u}^{n}(s)\right), w\right)_{L^{2}}-\left(\operatorname{Div}\left(h u^{*}(s)\right), w\right)_{L^{2}}\right) d s\right|\right]=0 .
$$

\section{Step III:}

Define for all $v \in \mathbb{H}_{0}^{1}(\mathcal{O})$

$$
\begin{aligned}
K^{1}\left(\bar{u}^{n}, \bar{z}^{n}, \bar{U}^{n}, \bar{N}^{n}, \bar{W}^{n}, v\right)(t)= & \left(\bar{u}_{0}^{n}, v\right)_{\mathbb{L}^{2}}+\left(\bar{U}^{n}, v\right)_{\mathbb{L}^{2}}-\int_{0}^{t}\left(A \bar{u}^{n}(s), v\right)_{\mathbb{L}^{2}} d s \\
& -\int_{0}^{t}\left(B\left(\bar{u}^{n}(s)\right), v\right)_{\mathbb{L}^{2}} d s-\int_{0}^{t}\left\langle g \nabla \bar{z}^{n}(s), v\right\rangle d s \\
& +\int_{0}^{t}\left(\sigma^{n}\left(s, \bar{u}^{n}(s)\right) d \bar{W}^{n}(s), v\right)_{\mathbb{L}^{2}}+\int_{0}^{t}(f(s), v)_{\mathbb{L}^{2}} d s \\
& +\int_{0}^{t} \int_{Z}\left(H^{n}\left(\bar{u}^{n}(s-), z\right), v\right)_{\mathbb{L}^{2}} \tilde{\bar{N}}^{n}(d s, d z),
\end{aligned}
$$

and for all $w \in H_{0}^{1}(\mathcal{O})$

$$
K^{2}\left(\bar{u}^{n}, \bar{z}^{n}, w\right)(t)=\left(\bar{z}_{0}^{n}, w\right)_{L^{2}}-\int_{0}^{t}\left(\operatorname{Div}\left(h \bar{u}^{n}(s)\right), w\right)_{L^{2}} d s .
$$

Hence for all $v \in \mathbb{H}_{0}^{1}(\mathcal{O})$

$$
\begin{aligned}
K^{1}\left(u^{*}, z^{*}, U^{*}, N^{*}, W^{*}, v\right)(t)= & \left(u_{0}^{*}, v\right)_{\mathbb{L}^{2}}+\left(U^{*}, v\right)_{\mathbb{L}^{2}}-\int_{0}^{t}\left(A u^{*}(s), v\right)_{\mathbb{L}^{2}} d s \\
& -\int_{0}^{t}\left(B\left(u^{*}(s)\right), v\right)_{\mathbb{L}^{2}} d s-\int_{0}^{t}\left\langle g \nabla z^{*}(s), v\right\rangle d s \\
& +\int_{0}^{t}\left(\sigma\left(s, u^{*}(s)\right) d W^{*}(s), v\right)_{\mathbb{L}^{2}}+\int_{0}^{t}(f(s), v)_{\mathbb{L}^{2}} d s \\
& +\int_{0}^{t} \int_{Z}\left(H\left(u^{*}(s-), z\right), v\right)_{\mathbb{L}^{2}} \tilde{N}^{*}(d s, d z),
\end{aligned}
$$

and for all $w \in H_{0}^{1}(\mathcal{O})$

$$
K^{2}\left(u^{*}, z^{*}, w\right)(t)=\left(z_{0}^{*}, w\right)_{L^{2}}-\int_{0}^{t}\left(\operatorname{Div}\left(h u^{*}(s)\right), w\right)_{L^{2}} d s .
$$

Claim 4. $\quad$ 1. For all $v \in \mathbb{H}_{0}^{1}(\mathcal{O})$

$$
\lim _{n \rightarrow \infty}\left\|\left(\bar{u}^{n}(\cdot), v\right)_{\mathbb{L}^{2}}-\left(u^{*}(\cdot), v\right)_{\mathbb{L}^{2}}\right\|_{L^{1}([0, T] \times \bar{\Omega})}=0,
$$

and

$\lim _{n \rightarrow \infty}\left\|K^{1}\left(\bar{u}^{n}, \bar{z}^{n}, \bar{U}^{n}, \bar{N}^{n}, \bar{W}^{n}, v\right)-K^{1}\left(u^{*}, z^{*}, U^{*}, N^{*}, W^{*}, v\right)\right\|_{L^{1}([0, T] \times \bar{\Omega})}=0$. 
2. For all $w \in H_{0}^{1}(\mathcal{O})$

$$
\lim _{n \rightarrow \infty}\left\|\left(\bar{z}^{n}(\cdot), w\right)_{L^{2}}-\left(z^{*}(\cdot), w\right)_{L^{2}}\right\|_{L^{1}([0, T] \times \bar{\Omega})}=0,
$$

and

$$
\lim _{n \rightarrow \infty}\left\|K^{2}\left(\bar{u}^{n}, \bar{z}^{n}, w\right)-K^{2}\left(u^{*}, z^{*}, w\right)\right\|_{L^{1}([0, T] \times \bar{\Omega})}=0 .
$$

\section{Proof of Claim 4.}

1. We note that (5.68) follows from Lemma 5.15(i). Now we see by Fubini's theorem,

$$
\begin{aligned}
& \left\|K^{1}\left(\bar{u}^{n}, \bar{z}^{n}, \bar{U}^{n}, \bar{N}^{n}, \bar{W}^{n}, v\right)-K^{1}\left(u^{*}, z^{*}, U^{*}, N^{*}, W^{*}, v\right)\right\|_{L^{1}([0, T] \times \bar{\Omega})} \\
& =\overline{\mathbb{E}}\left[\int_{0}^{T}\left|K^{1}\left(\bar{u}^{n}, \bar{z}^{n}, \bar{U}^{n}, \bar{N}^{n}, \bar{W}^{n}, v\right)-K^{1}\left(u^{*}, z^{*}, U^{*}, N^{*}, W^{*}, v\right)\right| d t\right] \\
& =\int_{0}^{T} \overline{\mathbb{E}}\left[\left|K^{1}\left(\bar{u}^{n}, \bar{z}^{n}, \bar{U}^{n}, \bar{N}^{n}, \bar{W}^{n}, v\right)-K^{1}\left(u^{*}, z^{*}, U^{*}, N^{*}, W^{*}, v\right)\right|\right] d t .
\end{aligned}
$$

Lemma 5.15 (ii)-(ix) ensure that each term on the right hand side of (5.64) converges to the right hand side of corresponding term in (5.66) in $L^{1}([0, T] \times \bar{\Omega})$ which further assures that right hand side of (5.72) goes to zero as $n \rightarrow \infty$. This verifies (5.69).

2. We note that (5.70) follows from Lemma $5.15(\mathrm{x})$. Now we see by Fubini's theorem,

$$
\begin{aligned}
& \left\|K^{2}\left(\bar{u}^{n}, \bar{z}^{n}, w\right)-K^{2}\left(u^{*}, z^{*}, w\right)\right\|_{L^{1}([0, T] \times \bar{\Omega})} \\
& =\overline{\mathbb{E}}\left[\int_{0}^{T}\left|K^{2}\left(\bar{u}^{n}, \bar{z}^{n}, w\right)-K^{2}\left(u^{*}, z^{*}, w\right)\right| d t\right] \\
& =\int_{0}^{T} \overline{\mathbb{E}}\left[\left|K^{2}\left(\bar{u}^{n}, \bar{z}^{n}, w\right)-K^{2}\left(u^{*}, z^{*}, w\right)\right|\right] d t .
\end{aligned}
$$

Lemma 5.15 (xi)-(xii) ensure that each term on the right hand side of (5.65) converges to the right hand side of corresponding term in (5.67) in $L^{1}([0, T] \times \bar{\Omega})$, which further assures that right hand side of (5.73) goes to zero as $n \rightarrow \infty$. This verifies (5.71). Hence our claim is established.

Step IV: Since $u^{n}$ is a solution of the Galerkin equation, we have for all $t \in[0, T]$

$$
\left(u^{n}(t), v\right)_{\mathbb{L}^{2}}=K^{1}\left(u^{n}, \hat{z}^{n}, U^{n}, N^{n}, W^{n}, v\right)(t) \quad P \text {-a.s. }
$$

Hence

$$
\int_{0}^{T} \mathbb{E}\left[\left|\left(u^{n}(t), v\right)_{\mathbb{L}^{2}}-K^{1}\left(u^{n}, \hat{z}^{n}, U^{n}, N^{n}, W^{n}, v\right)(t)\right|\right] d t=0 .
$$

Since $\mathcal{L}\left(\left(\bar{u}^{n}, \bar{z}^{n}, \bar{U}^{n}, \bar{N}^{n}, \bar{W}^{n}\right)\right)=\mathcal{L}\left(\left(u^{n}, \hat{z}^{n}, U^{n}, N^{n}, W^{n}\right)\right)$

$$
\int_{0}^{T} \overline{\mathbb{E}}\left[\left|\left(\bar{u}^{n}(t), v\right)_{\mathbb{L}^{2}}-K^{1}\left(\bar{u}^{n}, \bar{z}^{n}, \bar{U}^{n}, \bar{N}^{n}, \bar{W}^{n}, v\right)(t)\right|\right] d t=0 .
$$

Using (5.68), (5.74) and (5.69)

$$
\begin{aligned}
& \int_{0}^{T} \overline{\mathbb{E}}\left[\left|\left(u^{*}(t), v\right)_{\mathbb{L}^{2}}-K^{1}\left(u^{*}, z^{*}, U^{*}, N^{*}, W^{*}, v\right)(t)\right|\right] d t \\
& \leq \int_{0}^{T} \overline{\mathbb{E}}\left[\left|\left(u^{*}(t), v\right)_{\mathbb{L}^{2}}-\left(\bar{u}^{n}(t), v\right)_{\mathbb{L}^{2}}\right|\right] d t \\
& +\int_{0}^{T} \overline{\mathbb{E}}\left[\left|\left(\bar{u}^{n}(t), v\right)_{\mathbb{L}^{2}}-K^{1}\left(\bar{u}^{n}, \bar{z}^{n}, \bar{U}^{n}, \bar{N}^{n}, \bar{W}^{n}, v\right)(t)\right|\right] d t \\
& +\int_{0}^{T} \overline{\mathbb{E}}\left[\left|K^{1}\left(\bar{u}^{n}, \bar{z}^{n}, \bar{U}^{n}, \bar{N}^{n}, \bar{W}^{n}, v\right)(t)-K^{1}\left(u^{*}, z^{*}, U^{*}, N^{*}, W^{*}, v\right)(t)\right|\right] d t \\
& \rightarrow 0 \text { as } n \rightarrow \infty .
\end{aligned}
$$

Hence for almost all $t \in[0, T]$

$$
\left(u^{*}(t), v\right)_{\mathbb{L}^{2}}-K^{1}\left(u^{*}, z^{*}, U^{*}, N^{*}, W^{*}, v\right)(t)=0 \quad \bar{P} \text {-a.s. }
$$


Similarly we get for almost all $t \in[0, T]$

$$
\left(z^{*}(t), w\right)_{\mathbb{L}^{2}}-K^{2}\left(u^{*}, z^{*}, w\right)(t)=0 \quad \bar{P} \text {-a.s. }
$$

Taking $\bar{u}=u^{*}, \bar{z}=z^{*}, \bar{U}=U^{*}, \bar{N}=N^{*}$ and $\bar{W}=W^{*}$. we see that $(\bar{\Omega}, \overline{\mathcal{F}}, \bar{F}, \bar{P}, \bar{u}, \bar{z}, \bar{U}, \bar{N}, \bar{W})$ is a martingale solution of (5.4)-(5.6).

Existence of martingale solution (see Theorem 5.13) and pathwise uniqueness (see Step V of the proof of Theorem [1.1) guarantee uniqueness in law due to the classical Yamada-Watanabe technique [52] (see also Theorems 2 and 11 in Ondreját 39] for the infinite dimensional version of the result).

5.4. Existence of Optimal Control. This Subsection has been developed using certain ideas from Brzeźniak and Serrano [12, Sritharan [44]. Let $\left(\Omega, \mathcal{F}, \mathcal{F}_{t}, P\right)$ be a given filtered probability space. The objective of this subsection is to study optimal initial value control problem (5.4)-(5.6) of minimizing a finite-horizon cost functional of the form

$$
\mathcal{J}(u, \hat{z}, U)=\mathbb{E}\left[\int_{0}^{T} \int_{\mathcal{O}} L(t, u(t, x), \hat{z}(t, x), U(x)) d x d t\right] .
$$

Definition 5.16. A function $\kappa: \mathbb{L}^{2}(\mathcal{O}) \rightarrow[0,+\infty]$ is called inf-compact iff for every $R \geq 0$ the level set $\{\kappa \leq R\}$ is compact.

Assumption 5.17. Let the running cost function $L(\cdot, \cdot, \cdot, \cdot, \cdot):[0, T] \times \mathbb{H}_{0}^{1}(\mathcal{O}) \times L^{2}(\mathcal{O}) \times \mathbb{L}^{2}(\mathcal{O}) \times$ $\Omega \rightarrow \mathbb{R}$ be such that

(i) $L(\cdot, \cdot, \cdot, \cdot, \cdot)$ is measurable,

(ii) $L(t, \cdot, \cdot, \cdot, \omega): \mathbb{H}_{0}^{1}(\mathcal{O})_{w} \times L^{2}(\mathcal{O}) \times \mathbb{L}^{2}(\mathcal{O})$ is lower semicontinuous $\forall t \in[0, T]$ and $w \in \Omega$, where $\mathbb{H}_{0}^{1}(\mathcal{O})_{w}$ is the space $\mathbb{H}_{0}^{1}(\mathcal{O})$ endowed with the weak topology.

(iii) There exists an inf-compact function $\kappa: \mathbb{L}^{2}(\mathcal{O}) \rightarrow[0,+\infty]$ such that for every $(t, u, \hat{z}, U) \in$ $[0, T] \times \mathbb{H}_{0}^{1}(\mathcal{O}) \times L^{2}(\mathcal{O}) \times \mathbb{L}^{2}(\mathcal{O}), L(t, u, \hat{z}, U) \geq \kappa^{2}(U), P$-a.s., and $\|\kappa(U)\|_{L^{2}\left(\Omega ; \mathbb{L}^{2}\right)} \rightarrow \infty$ as $\|U\|_{L^{2}\left(\Omega ; \mathbb{L}^{2}\right)} \rightarrow \infty$.

A specific example of cost functional is as follows:

$$
\mathcal{J}(u, \hat{z}, U)=\mathbb{E}\left[\int_{0}^{T}\left(\|u(s)\|_{\mathbb{H}_{0}^{1}}^{2}+\|\hat{z}(s)\|_{L^{2}}^{2}\right) d s\right]+\mathbb{E}\|U\|_{\mathbb{L}^{2}}^{2} .
$$

Definition 5.18. Let $u_{0} \in L^{2}\left(\Omega ; \mathbb{L}^{2}(\mathcal{O})\right)$ and $\hat{z}_{0} \in L^{2}\left(\Omega ; L^{2}(\mathcal{O})\right)$ and let $T>0$ be fixed. A weak admissible control (with time horizon $[0, T]$ ) is a system

$$
\pi=\left(\Omega, \mathcal{F}, \mathcal{F}_{t}, P,\{W(t)\}_{t \geq 0},\{N(t, \cdot)\}_{t \geq 0},\{u(t)\}_{t \geq 0},\{\hat{z}(t)\}_{t \geq 0}, U\right)
$$

such that

1. $(\Omega, \mathcal{F}, F, P)$ is a filtered probability space with a filtration $F=\left\{\mathcal{F}_{t}\right\}_{t \geq 0}$,

2. $N$ is a time homogeneous Poisson random measure over $(\Omega, \mathcal{F}, F, P)$ with the intensity measure $\lambda$,

3. $W$ is a cylindrical Wiener process over $(\Omega, \mathcal{F}, F, P)$,

4. $U$ is measurable with $P$ - a.e. $\omega \in \Omega, U(\omega) \in \mathbb{L}^{2}(\mathcal{O})$,

5. $u, z$ are progressively measurable processes with $P$-a.e. $\omega \in \Omega$, the paths

$$
\begin{aligned}
& u(\cdot, \omega) \in D\left([0, T] ; \mathbb{H}^{-1}(\mathcal{O})\right) \cap D\left([0, T] ; \mathbb{L}_{w}^{2}(\mathcal{O})\right) \cap L_{w}^{2}\left(0, T ; \mathbb{H}_{0}^{1}(\mathcal{O})\right) \cap L^{2}\left(0, T ; \mathbb{L}^{2}(\mathcal{O})\right), \\
& \hat{z}(\cdot, \omega) \in L^{2}\left(0, T ; L^{2}(\mathcal{O})\right) \cap C\left([0, T] ; H^{-1}(\mathcal{O})\right)
\end{aligned}
$$

such that for all $t \in[0, T]$, for all $v \in \mathbb{H}_{0}^{1}(\mathcal{O})$ and for all $w \in L^{2}(\mathcal{O})$, the following identities hold $P$-a.s.

$$
\begin{aligned}
& (u(t), v)_{\mathbb{L}^{2}}+\int_{0}^{t}(A u(s), v)_{\mathbb{L}^{2}} d s+\int_{0}^{t}(B(u(s)), v)_{\mathbb{L}^{2}} d s+\int_{0}^{t}\langle g \nabla z(s), v\rangle d s \\
& =\left(u_{0}, v\right)_{\mathbb{L}^{2}}+(U, v)_{\mathbb{L}^{2}}+\int_{0}^{t}(f(s), v)_{\mathbb{L}^{2}} d s
\end{aligned}
$$




$$
\begin{gathered}
+\int_{0}^{t}(\sigma(s, u(s)) d W(s), v)_{\mathbb{L}^{2}}+\int_{0}^{t} \int_{Z}(H(u(s-), z), v)_{\mathbb{L}^{2}} \tilde{N}(d s, d z), \\
(\hat{z}(t), w)_{L^{2}}+\int_{0}^{t}(\operatorname{Div}(h u(s)), w)_{L^{2}} d s=\left(\hat{z}_{0}, w\right)_{L^{2}},
\end{gathered}
$$

6. the mapping

$$
[0, T] \times \mathcal{O} \times \Omega \ni(t, x, \omega) \mapsto L(t, u(t, x, \omega), \hat{z}(t, x, \omega), U(x, \omega)) \in \mathbb{R}
$$

belongs to $L^{1}([0, T] \times \mathcal{O} \times \Omega ; \mathbb{R})$.

In the spirit of Definition [5.9, from 1-4 of Definition [5.18, we note that $\pi$ is a martingale solution of (5.4) -(5.6) associated with (5.75). The set of weak admissible controls (with time horizon $[0, T]$ ) will be denoted by $\overline{\mathcal{U}}_{a d}^{w}\left(u_{0}, \hat{z}_{0}, T\right)$. In this context, under this weak formulation, the cost functional is defined as

$$
J(\pi):=\mathbb{E}\left[\int_{0}^{T} \int_{\mathcal{O}} L(t, u, \hat{z}, U) d x d t\right], \quad \pi \in \overline{\mathcal{U}}_{a d}^{w}\left(u_{0}, \hat{z}_{0}, T\right)
$$

where $\pi$ has the form (5.77) and $(u, \hat{z}, U)$ are the components of $\pi$.

Remark 5.19. The optimal control problem is to minimize $J$ over $\overline{\mathcal{U}}_{a d}^{w}\left(u_{0}, \hat{z}_{0}, T\right)$ for $u_{0} \in L^{2}\left(\Omega ; \mathbb{L}^{2}(\mathcal{O})\right)$ and $\hat{z}_{0} \in L^{2}\left(\Omega ; L^{2}(\mathcal{O})\right)$ and $T>0$ be fixed. Namely, we seek $\tilde{\pi} \in \overline{\mathcal{U}}_{a d}^{w}\left(u_{0}, \hat{z}_{0}, T\right)$ such that

$$
J(\tilde{\pi})=\inf _{\pi \in \overline{\mathcal{U}}_{a d}^{w}\left(u_{0}, \hat{z}_{0}, T\right)} J(\pi) .
$$

We now prove Theorem 1.2, the main result in this Subsection which guarantees the existence of weak optimal control.

\section{Proof of Theorem 1.2,}

Proof. Step I: From the assumptions of the Theorem 1.2, we have $u_{0} \in L^{2}\left(\Omega ; \mathbb{L}^{2}(\mathcal{O})\right)$ and $\hat{z}_{0} \in L^{2}\left(\Omega ; L^{2}(\mathcal{O})\right)$ and $J(\pi)<+\infty$. Since $J$ is bounded below by zero on $\overline{\mathcal{U}}_{a d}^{w}\left(u_{0}, \hat{z}_{0}, T\right)$, there exists a minimizing sequence $\left\{\pi^{n}\right\}_{n \geq 1}$ such that $J\left(\pi^{n}\right) \rightarrow \inf _{\pi \in \overline{\mathcal{U}}_{a d}^{w}\left(u_{0}, \hat{z}_{0}, T\right)} J(\pi)$. In other words, $\pi^{n}=$ $\left(\Omega^{n}, \mathcal{F}^{n}, \mathcal{F}_{t}^{n}, P^{n},\left\{W^{n}(t)\right\}_{t \geq 0},\left\{N^{n}(t, \cdot)\right\}_{t \geq 0},\left\{u^{n}(t)\right\}_{t \geq 0},\left\{\hat{z}^{n}(t)\right\}_{t \geq 0}, U^{n}\right)$ is a minimizing sequence of weak admissible controls, that is,

$$
\lim _{n \rightarrow \infty} J\left(\pi^{n}\right)=\inf _{\pi \in \overline{\mathcal{U}}_{a d}^{w}\left(u_{0}, \hat{z}_{0}, T\right)} J(\pi) .
$$

Since for each $n \in \mathbb{N}, \pi^{n} \in \overline{\mathcal{U}}_{a d}^{w}\left(u_{0}, \hat{z}_{0}, T\right)$, hence each $\pi^{n}$ satisfies

$$
\begin{aligned}
& \left(u^{n}(t), v\right)_{\mathbb{L}^{2}}+\int_{0}^{t}\left(A u^{n}(s), v\right)_{\mathbb{L}^{2}} d s+\int_{0}^{t}\left(B\left(u^{n}(s)\right), v\right)_{\mathbb{L}^{2}} d s+\int_{0}^{t}\left\langle g \nabla \hat{z}^{n}(s), v\right\rangle d s \\
& =\left(u_{0}, v\right)_{\mathbb{L}^{2}}+\left(U^{n}, v\right)_{\mathbb{L}^{2}}+\int_{0}^{t}(f(s), v)_{\mathbb{L}^{2}} d s \\
& \quad+\int_{0}^{t}\left(\sigma(s, u(s)) d W^{n}(s), v\right)_{\mathbb{L}^{2}}+\int_{0}^{t} \int_{Z}(H(u(s-), z), v)_{\mathbb{L}^{2}} \tilde{N}^{n}(d s, d z), \\
& \left(\hat{z}^{n}(t), w\right)_{L^{2}}+\int_{0}^{t}\left(\operatorname{Div}\left(h u^{n}(s)\right), w\right)_{L^{2}} d s=\left(\hat{z}_{0}, w\right)_{L^{2}} .
\end{aligned}
$$

This holds $P^{n}$-a.s. for almost all $t \in[0, T]$, for all $v \in \mathbb{H}_{0}^{1}(\mathcal{O})$ and for all $w \in L^{2}(\mathcal{O})$. Proceeding as in similar lines as in Propositions 4.3 4.4, we have the following a-priori estimates (uniformly in $n$ )

$$
\begin{aligned}
& \sup _{n \geq 1} \mathbb{E}^{n}\left[\sup _{0 \leq s \leq T}\left\|u^{n}(s)\right\|_{\mathbb{L}^{2}}^{2}\right] \leq C, \quad \sup _{n \geq 1} \mathbb{E}^{n}\left[\int_{0}^{T}\left\|u^{n}(s)\right\|_{\mathbb{H}_{0}^{1}}^{2} d s\right] \leq C, \\
& \text { and } \quad \sup _{n \geq 1} \mathbb{E}^{n}\left[\sup _{0 \leq s \leq T}\left\|\hat{z}^{n}(s)\right\|_{L^{2}}^{2}\right] \leq C,
\end{aligned}
$$


where $\mathbb{E}^{n}$ denotes the expectation with respect to $P^{n}$. Also we note from (5.81) that there exists a constant $C>0$ such that $J\left(\pi^{n}\right) \leq C$. Using Assumption 5.17 (iii), (5.75) we have

$$
\begin{aligned}
\left\|\kappa\left(U^{n}\right)\right\|_{L^{2}\left(\Omega ; \mathbb{L}^{2}\right)}^{2} & =\frac{1}{T} \mathbb{E}^{n}\left[\int_{0}^{T} \int_{\mathcal{O}} \kappa^{2}\left(U^{n}\right) d x d t\right] \leq \frac{1}{T} \mathbb{E}^{n}\left[\int_{0}^{T} \int_{\mathcal{O}} L\left(t, u^{n}, \hat{z}^{n}, U^{n}\right) d x d t\right] \\
& =\frac{1}{T} J\left(\pi^{n}\right) \leq C_{T} .
\end{aligned}
$$

In view of (5.86) and Assumption 5.17 (iii) we have

$$
\sup _{n \geq 1} \mathbb{E}^{n}\left[\left\|U^{n}\right\|_{\mathbb{L}^{2}}^{2}\right] \leq C_{T} .
$$

Due to the uniform a-priori bounds ([5.84), one can establish that $\left\{\mathcal{L}\left(u^{n}\right), n \in \mathbb{N}\right\}$ is tight on $(\mathcal{Z}, \tau)$ by first proving the Aldous condition (as in Lemma 5.10) and then employing Theorem 5.8. Similarly, due to uniform bounds (5.85) and (5.87), we can prove (as in Lemmas 5.11 15.12) that the set of measures $\left\{\mathcal{L}\left(\hat{z}^{n}, U^{n}\right), n \in \mathbb{N}\right\}$ is tight on $\left(L^{2}\left(0, T ; L^{2}(\mathcal{O})\right) \cap C\left([0, T] ; H^{-1}(\mathcal{O})\right)\right) \times \mathbb{L}^{2}(\mathcal{O})$. Therefore by the Skorokhod theorem, there exists a subsequence $\left(n_{k}\right)_{k \in \mathbb{N}}$, a probability space $(\tilde{\Omega}, \tilde{\mathcal{F}}, \tilde{P})$, and, on this space, random variables $(\tilde{u}, \tilde{\hat{z}}, \tilde{U}, \breve{N}, \tilde{W}),\left(\tilde{u}^{k}, \tilde{\hat{z}}^{k}, \tilde{U}^{k}, \breve{N}^{k}, \tilde{W}^{k}\right)_{k \in \mathbb{N}}$ such that

(i) $\mathcal{L}\left(\left(\tilde{u}^{k}, \tilde{z}^{k}, \tilde{U}^{k}, \breve{N}^{k}, \tilde{W}^{k}\right)\right)=\mathcal{L}\left(\left(u^{n_{k}}, \hat{z}^{n_{k}}, U^{n_{k}}, N^{n_{k}}, W^{n_{k}}\right)\right)$ for all $k \in \mathbb{N}$,

(ii) $\left(\tilde{u}^{k}, \tilde{\hat{z}}^{k}, \tilde{U}^{k}, \breve{N}^{k}, \tilde{W}^{k}\right) \rightarrow(\tilde{u}, \tilde{\hat{z}}, \tilde{U}, \breve{N}, \tilde{W})$ in $\mathcal{Z} \times\left(L^{2}\left(0, T ; L^{2}(\mathcal{O})\right) \cap C\left([0, T] ; H^{-1}(\mathcal{O})\right)\right) \times$ $\mathbb{L}^{2}(\mathcal{O}) \times M_{\overline{\mathbb{N}}}([0, T] \times Z) \times C([0, T] ; \mathbb{R})$ with probability 1 on $(\tilde{\Omega}, \tilde{\mathcal{F}}, \tilde{P})$ as $k \rightarrow \infty$,

(iii) $\left(\breve{N}^{k}(\tilde{\omega}), \tilde{W}^{k}(\tilde{\omega})\right)=(\breve{N}(\tilde{\omega}), \tilde{W}(\tilde{\omega}))$ for all $\tilde{\omega} \in \tilde{\Omega}$.

For convenience, we denote these sequences again by $\left(\left(u^{n}, \hat{z}^{n}, U^{n}, N^{n}, W^{n}\right)\right)_{n \in \mathbb{N}}$ and $\left(\left(\tilde{u}^{n}, \tilde{\hat{z}}^{n}, \tilde{U}^{n}\right.\right.$, $\left.\left.\breve{N}^{n}, \tilde{W}^{n}\right)\right)_{n \in \mathbb{N}}$. Now, following the similar steps as in Theorem 5.13, one can establish that

$$
\tilde{\pi}=\left(\tilde{\Omega}, \tilde{\mathcal{F}}, \tilde{\mathcal{F}}_{t}, \tilde{P},\{\tilde{W}(t)\}_{t \geq 0},\{\tilde{N}(t, \cdot)\}_{t \geq 0},\{\tilde{u}(t)\}_{t \geq 0},\{\tilde{\tilde{z}}(t)\}_{t \geq 0}, \tilde{U}\right)
$$

is a martingale solution of (5.4)-(5.6) associated to (5.75).

\section{Step II:}

We now prove that the cost functional is lower semicontinuous. Below we extend the proof of Lemma 10 and Lemma 11 of Sritharan [4] to the stochastic case.

Lemma 5.20. For $\tilde{u}^{n} \rightarrow \tilde{u}$ in the $L^{2}(\tilde{\Omega} ; \mathcal{Z}), \tilde{z}^{n} \rightarrow \tilde{\tilde{z}}$ in $L^{2}\left(\tilde{\Omega} ; L^{2}\left(0, T ; L^{2}(\mathcal{O})\right)\right.$ and $\tilde{U}^{n} \rightarrow \tilde{U}$ in $L^{2}\left(\tilde{\Omega} ; \mathbb{L}^{2}(\mathcal{O})\right)$, we have

$$
\liminf _{n \rightarrow \infty} \tilde{\mathbb{E}}\left[\int_{0}^{T} \int_{\mathcal{O}} L\left(t, \tilde{u}^{n}, \tilde{\hat{z}}^{n}, \tilde{U}^{n}\right) d x d t\right] \geq \tilde{\mathbb{E}}\left[\int_{0}^{T} \int_{\mathcal{O}} L(t, \tilde{u}, \tilde{\hat{z}}, \tilde{U}) d x d t\right] .
$$

Proof. For each natural number $M$, let $L_{M}(t, \tilde{u}, \tilde{\hat{z}}, \tilde{U})=L(t, \tilde{u}, \tilde{\hat{z}}, \tilde{U}) \wedge M$. Then by linearity of expectation, we have

$$
\begin{aligned}
& \liminf _{n \rightarrow \infty} \tilde{\mathbb{E}}\left[\int_{0}^{T} \int_{\mathcal{O}} L\left(t, \tilde{u}^{n}, \tilde{z}^{n}, \tilde{U}^{n}\right) d x d t\right] \\
& \geq \liminf _{n \rightarrow \infty} \tilde{\mathbb{E}}\left[\int_{0}^{T} \int_{\mathcal{O}} L_{M}\left(t, \tilde{u}^{n}, \tilde{z}^{n}, \tilde{U}^{n}\right) d x d t\right] \\
& \geq-\limsup _{n \rightarrow \infty} \tilde{\mathbb{E}}\left[\int_{0}^{T} \int_{\mathcal{O}}\left(L_{M}\left(t, \tilde{u}^{n}, \tilde{\hat{z}}^{n}, \tilde{U}^{n}\right)-L_{M}\left(t, \tilde{u}, \tilde{\hat{z}}^{n}, \tilde{U}^{n}\right)\right)^{-} d x d t\right] \\
& \quad-\limsup _{n \rightarrow \infty} \tilde{\mathbb{E}}\left[\int_{0}^{T} \int_{\mathcal{O}}\left(L_{M}\left(t, \tilde{u}, \tilde{\hat{z}}^{n}, \tilde{U}^{n}\right)-L_{M}\left(t, \tilde{u}, \tilde{\hat{z}}, \tilde{U}^{n}\right)\right)^{-} d x d t\right] \\
& \quad+\liminf _{n \rightarrow \infty} \tilde{\mathbb{E}}\left[\int_{0}^{T} \int_{\mathcal{O}} L_{M}\left(t, \tilde{u}, \tilde{z}, \tilde{U}^{n}\right) d x d t\right],
\end{aligned}
$$


where $f(x)^{-}=(-f(x)) \vee 0$. The first and second terms on the right-hand side are zero due to Lemma 5.21 given below. Due to the lower semicontinuity of $L_{M}$, from Jacod and Mémin [23], Lemma 4 of Sritharan [44, Proposition 2.1.12 of Castaing et al. [13] we have

$$
\liminf _{n \rightarrow \infty}\left[\int_{0}^{T} \int_{\mathcal{O}} L_{M}\left(t, \tilde{u}, \tilde{\tilde{z}}, \tilde{U}^{n}\right) d x d t\right] \geq\left[\int_{0}^{T} \int_{\mathcal{O}} L_{M}(t, \tilde{u}, \tilde{\tilde{z}}, \tilde{U}) d x d t\right] .
$$

Therefore application of Fatou's Lemma gives

$$
\liminf _{n \rightarrow \infty} \tilde{\mathbb{E}}\left[\int_{0}^{T} \int_{\mathcal{O}} L_{M}\left(t, \tilde{u}, \tilde{\hat{z}}, \tilde{U}^{n}\right) d x d t\right] \geq \tilde{\mathbb{E}}\left[\int_{0}^{T} \int_{\mathcal{O}} L_{M}(t, \tilde{u}, \tilde{\tilde{z}}, \tilde{U}) d x d t\right] .
$$

Hence using the Beppo-Levi theorem on the bounded measurable functions $L_{M}$ we have

$$
\begin{aligned}
\liminf _{n \rightarrow \infty} \tilde{\mathbb{E}}\left[\int_{0}^{T} \int_{\mathcal{O}} L\left(t, \tilde{u}^{n}, \tilde{\hat{z}}^{n}, \tilde{U}^{n}\right) d x d t\right] & \geq \tilde{\mathbb{E}}\left[\int_{0}^{T} \int_{\mathcal{O}} L_{M}(t, \tilde{u}, \tilde{\hat{z}}, \tilde{U}) d x d t\right] \\
& \geq \tilde{\mathbb{E}}\left[\int_{0}^{T} \int_{\mathcal{O}} L(t, \tilde{u}, \tilde{\tilde{z}}, \tilde{U}) d x d t\right]
\end{aligned}
$$

Lemma 5.21. Let $\tilde{u}^{n} \rightarrow \tilde{u}$ in the $L^{2}(\tilde{\Omega} ; \mathcal{Z}), \tilde{\tilde{z}}^{n} \rightarrow \tilde{\hat{z}}$ in $L^{2}\left(\tilde{\Omega} ; L^{2}\left(0, T ; L^{2}(\mathcal{O})\right)\right.$ and $\tilde{U}^{n} \rightarrow \tilde{U}$ in $L^{2}\left(\tilde{\Omega} ; \mathbb{L}^{2}(\mathcal{O})\right)$. For $\tilde{\omega} \in \tilde{\Omega}$, let $\varphi(\cdot, \cdot, \cdot, \cdot, \tilde{\omega}):[0, T] \times \mathbb{H}_{0}^{1}(\mathcal{O}) \times L^{2}(\mathcal{O}) \times \mathbb{L}^{2}(\mathcal{O}) \rightarrow \mathbb{R}_{+}$be a bounded measurable function such that $\forall t \in(0, T)$ and $\tilde{\omega} \in \tilde{\Omega}, \varphi(t, \cdot, \cdot, \cdot, \tilde{\omega}): \mathbb{H}_{0}^{1}(\mathcal{O}) \times L^{2}(\mathcal{O}) \times \mathbb{L}^{2}(\mathcal{O}) \rightarrow \mathbb{R}_{+}$ is lower semicontinuous. Then

$$
\lim _{n \rightarrow \infty} \tilde{\mathbb{E}}\left[\int_{0}^{T} \int_{\mathcal{O}}\left(\varphi\left(t, \tilde{u}^{n}, \tilde{\hat{z}}^{n}, \tilde{U}^{n}\right)-\varphi\left(t, \tilde{u}, \tilde{\hat{z}}^{n}, \tilde{U}^{n}\right)\right)^{-} d x d t\right]=0,
$$

and

$$
\lim _{n \rightarrow \infty} \tilde{\mathbb{E}}\left[\int_{0}^{T} \int_{\mathcal{O}}\left(\varphi\left(t, \tilde{u}, \tilde{z}^{n}, \tilde{U}^{n}\right)-\varphi\left(t, \tilde{u}, \tilde{\hat{z}}, \tilde{U}^{n}\right)\right)^{-} d x d t\right]=0 .
$$

Proof. Define, $\Theta(t, \tilde{v}, \tilde{\hat{z}}, \tilde{U}, \tilde{\omega}):=\varphi(t, \tilde{v}, \tilde{\hat{z}}, \tilde{U}, \tilde{\omega})-\varphi(t, \tilde{u}, \tilde{\hat{z}}, \tilde{U}, \tilde{\omega})$. For $\delta>0$ and $y \in \mathbb{H}^{-1}(\mathcal{O})$, define

$$
\begin{aligned}
& Y^{m}:=\{(t, x, \tilde{\omega}) \in[0, T] \times \mathcal{O} \times \tilde{\Omega}: \\
& \left.\inf _{|\langle y, \tilde{u}(t, x)\rangle-\langle y, \tilde{v}(t, x)\rangle| \leq 1 / m} \Theta(t, \tilde{v}(t, x), \tilde{\tilde{z}}(t, x), \tilde{U}(x), \tilde{\omega}) \leq-\delta\right\} .
\end{aligned}
$$

Note that $Y^{m+1} \subseteq Y^{m}$, and lower semicontinuity of $\varphi$ implies that each $t$-section of $Y^{m}$ is closed. Moreover, $\varphi(t, \cdot \tilde{\tilde{z}}, \tilde{U}, \tilde{\omega}): \mathbb{H}_{0}^{1}(\mathcal{O}) \rightarrow \mathbb{R}_{+}$being lower semicontinuous implies that if for any sequence $\tilde{v}^{n} \rightarrow \tilde{u}$ in $\mathbb{H}_{0}^{1}(\mathcal{O})$, we have

$$
\liminf _{n \rightarrow \infty} \Theta\left(t, \tilde{v}^{n}, \tilde{\hat{z}}, \tilde{U}, \tilde{\omega}\right) \geq 0
$$

Hence

$$
\cap_{m} Y^{m}=\emptyset
$$

Now we define

$$
\begin{aligned}
\hat{Y}^{n}: & =\left\{(t, x, \tilde{\omega}): \Theta\left(t, \tilde{u}^{n}(t, x), \tilde{\hat{z}}^{n}(t, x), \tilde{U}^{n}(x), \tilde{\omega}\right)^{-}>\delta\right\} \\
& =\left\{(t, x, \tilde{\omega}): \Theta\left(t, \tilde{u}^{n}(t, x), \tilde{\hat{z}}^{n}(t, x), \tilde{U}^{n}(x), \tilde{\omega}\right)<-\delta\right\} .
\end{aligned}
$$

Then for sufficiently large $n, \tilde{P}\left(\hat{Y}^{n}\right) \leq \tilde{P}\left(Y^{m}\right)$ (as $\hat{Y}^{n} \subseteq Y^{m}$ for $n>m$ ). Furthermore,

$$
\limsup _{n \rightarrow \infty} \tilde{P}\left(\hat{Y}^{n}\right)=\lim _{m \rightarrow \infty} \limsup _{n \rightarrow \infty} \tilde{P}\left(\hat{Y}^{n}\right) \leq \lim _{m \rightarrow \infty} \tilde{P}\left(Y^{m}\right)=\tilde{P}\left(\cap_{m} Y^{m}\right)=0 .
$$


We now proceed as in Jacod and Mémin 23 to obtain (5.89). Since $\varphi$ 's are bounded measurable, so $\left|\Theta\left(t, \tilde{u}^{n}, \tilde{\hat{z}}^{n}, \tilde{U}^{n}, \tilde{\omega}\right)\right| \leq C \forall n$, and using (5.91) we have

$$
\begin{aligned}
& \int_{\tilde{\Omega}} \int_{0}^{T} \int_{\mathcal{O}} \Theta\left(t, \tilde{u}^{n}(t, x), \tilde{\hat{z}}^{n}(t, x), \tilde{U}^{n}(x), \tilde{\omega}\right)^{-} d x d t d \tilde{P}(\tilde{\omega}) \\
& =\int_{\hat{Y}^{\prime}} \Theta\left(t, \tilde{u}^{n}(t, x), \tilde{z}^{n}(t, x), \tilde{U}^{n}(x), \tilde{\omega}\right)^{-} d x d t d \tilde{P}(\tilde{\omega}) \\
& \quad+\int_{\hat{Y}^{n}} \Theta\left(t, \tilde{u}^{n}(t, x), \tilde{\hat{z}}^{n}(t, x), \tilde{U}^{n}(x), \tilde{\omega}\right)^{-} d x d t d \tilde{P}(\tilde{\omega}) \\
& <\delta \tilde{P}\left(\hat{Y}^{\prime}\right)+C \tilde{P}\left(\hat{Y}^{n}\right) \rightarrow 0, \quad \text { as } n \rightarrow \infty
\end{aligned}
$$

and as $\delta>0$ is arbitrarily small where $\hat{Y}^{\prime}{ }^{n}$ denotes the complement of $\hat{Y}^{n}$. Therefore

$$
\lim _{n \rightarrow \infty} \tilde{\mathbb{E}}\left[\int_{0}^{T} \int_{\mathcal{O}} \Theta\left(t, \tilde{u}^{n}, \tilde{\hat{z}}^{n}, \tilde{U}^{n}\right)^{-} d x d t\right]=0,
$$

which proves (5.89). By similar approach one can prove (5.90).

Step III:

Using Lemma 5.20 and using $\mathcal{L}\left(\tilde{u}^{n}, \tilde{\hat{z}}^{n}, \tilde{U}^{n}\right)=\mathcal{L}\left(u^{n}, \hat{z}^{n}, U^{n}\right)$, we have

$$
\begin{aligned}
\tilde{\mathbb{E}}\left[\int_{0}^{T} \int_{\mathcal{O}} L(t, \tilde{u}(t), \tilde{z}(t), \tilde{U}) d x d t\right] & \leq \liminf _{n \rightarrow \infty} \tilde{\mathbb{E}}\left[\int_{0}^{T} \int_{\mathcal{O}} L\left(t, \tilde{u}^{n}(t), \tilde{z}^{n}(t), \tilde{U}^{n}\right) d x d t\right] \\
& =\liminf _{n \rightarrow \infty} \mathbb{E}^{n}\left[\int_{0}^{T} \int_{\mathcal{O}} L\left(t, u^{n}(t), \hat{z}^{n}(t), U^{n}\right) d x d t\right]
\end{aligned}
$$

which is finite because of (5.81) and $J(\pi)<+\infty$, where $\mathbb{E}^{n}$ denotes the expectation with respect to $P^{n}$. Hence, $J(\tilde{\pi})<+\infty$, or in other words, the mapping

$$
[0, T] \times \mathcal{O} \times \tilde{\Omega} \ni(t, x, \tilde{\omega}) \mapsto L(t, \tilde{u}(t, x, \tilde{\omega}), \tilde{\hat{z}}(t, x, \tilde{\omega}), \tilde{U}(x, \tilde{\omega})) \in \mathbb{R}
$$

belongs to $L^{1}([0, T] \times \mathcal{O} \times \tilde{\Omega} ; \mathbb{R})$. Therefore, $\tilde{\pi}$ satisfies all the conditions of Definition $[5.18$ and hence is a weak admissible control.

Now employing (5.81), (5.92) and $\mathcal{L}\left(\tilde{u}^{n}, \tilde{\hat{z}}^{n}, \tilde{U}^{n}\right)=\mathcal{L}\left(u^{n}, \hat{z}^{n}, U^{n}\right)$, it follows that

$$
\begin{aligned}
J(\tilde{\pi}) & =\tilde{\mathbb{E}}\left[\int_{0}^{T} \int_{\mathcal{O}} L(t, \tilde{u}(t), \tilde{\tilde{z}}(t), \tilde{U}) d x d t\right] \\
& \leq \liminf _{n \rightarrow \infty}\left[\mathbb{E}^{n} \int_{0}^{T} \int_{\mathcal{O}} L\left(t, u^{n}(t), \hat{z}^{n}(t), U^{n}\right) d x d t\right] \\
& =\inf _{\pi \in \overline{\mathcal{U}}_{a d}^{w}\left(u_{0}, \hat{z}_{0}, T\right)} J(\pi) .
\end{aligned}
$$

This proves $\tilde{\pi}$ is a weak optimal control for the control problem, hence this completes the proof.

\section{Appendix A.}

Here we will provide a proof of $\mathbb{H}_{0}^{1}$ energy estimate of (4.1)-(4.3). For this we need to impose more regular conditions on the noise coefficients $\sigma$ and $H$. We assume that $\sigma$ and $H$ satisfy the following hypotheses:

A.1 $\sigma \in C\left([0, T] \times \mathbb{H}_{0}^{1}(\mathcal{O}) ; L_{Q}\left(\mathbb{L}^{2}, \mathbb{H}_{0}^{1}\right)\right), H \in \mathbb{H}_{\lambda}^{2}\left([0, T] \times Z ; \mathbb{H}_{0}^{1}(\mathcal{O})\right)$,

A.2 For all $t \in(0, T)$, there exists a positive constant $K$ such that for all $u \in \mathbb{H}_{0}^{1}(\mathcal{O})$

$$
\|\sigma(t, u)\|_{L_{Q}\left(\mathbb{L}^{2}, \mathbb{H}_{0}^{1}\right)}^{2}+\int_{Z}\|H(u, z)\|_{\mathbb{H}_{0}^{1}}^{2} \lambda(d z) \leq K\left(1+\|u\|_{\mathbb{H}_{0}^{1}}^{2}\right),
$$

A.3 For all $t \in(0, T)$, there exists a positive constant $L$ such that for all $u, v \in \mathbb{H}_{0}^{1}(\mathcal{O})$

$$
\|\sigma(t, u)-\sigma(t, v)\|_{L_{Q}\left(\mathbb{L}^{2}, \mathbb{H}_{0}^{1}\right)}^{2}+\int_{Z}\|H(u, z)-H(v, z)\|_{\mathbb{H}_{0}^{1}}^{2} \lambda(d z) \leq L\|u-v\|_{\mathbb{H}_{0}^{1}}^{2} .
$$

We also make two additional assumptions, due to technical reasons, as following: 
A.4 The depth function $h \in C_{b}^{2}(\mathcal{O})$.

A.5 curl curlu $=0$ (i.e., rotation of the fluid velocity is uniform over $\mathcal{O}$ ).

Note that, in two dimensions curlu is a scalar that should be understood as follows

$$
\text { curlu }:=\nabla \times u=\nabla \times\left(u_{1} e_{1}+u_{2} e_{2}+0 e_{3}\right)=0 e_{1}+0 e_{2}+\left(\frac{\partial u_{2}}{\partial x}-\frac{\partial u_{1}}{\partial y}\right) e_{3},
$$

where $\left(e_{1}, e_{2}, e_{3}\right)$ is the canonical basis of $\mathbb{R}^{3}$.

\section{Proposition A.1. Let}

$$
\left\{\begin{array}{l}
w^{0} \in L^{4}\left(\Omega ; L^{4}\left(0, T ; \mathbb{H}_{0}^{1}(\mathcal{O})\right)\right), f \in L^{2}\left(\Omega ; L^{2}\left(0, T ; \mathbb{H}_{0}^{1}(\mathcal{O})\right)\right) \\
u_{0} \in L^{2}\left(\Omega ; \mathbb{H}_{0}^{1}(\mathcal{O})\right), \hat{z}_{0} \in L^{2}\left(\Omega ; H_{0}^{1}(\mathcal{O})\right)
\end{array}\right.
$$

and the assumptions A.1 - A.5 hold. If $\left(u^{n}(t), \hat{z}^{n}(t)\right)$ denotes the unique strong solution of the system (4.1)-(4.3), then the following a-priori estimates hold:

$$
\begin{aligned}
& \mathbb{E}\left[\left\|u^{n}(t)\right\|_{\mathbb{H}_{0}^{1}}^{2}+\left\|\hat{z}^{n}(t)\right\|_{\mathbb{H}_{0}^{1}}^{2}\right]+\frac{3 \alpha}{4} \mathbb{E} \int_{0}^{t}\left\|\Delta u^{n}(s)\right\|_{\mathbb{L}^{2}}^{2} d s \leq C_{11}, \quad \forall t \in[0, T], \\
& \mathbb{E}\left[\sup _{0 \leq t \leq T}\left(\left\|u^{n}(t)\right\|_{\mathbb{H}_{0}^{1}}^{2}+\left\|\hat{z}^{n}(t)\right\|_{\mathbb{H}_{0}^{1}}^{2}\right)\right]+\frac{3 \alpha}{4} \mathbb{E} \int_{0}^{T}\left\|\Delta u^{n}(t)\right\|_{\mathbb{L}^{2}}^{2} d t \leq C_{12},
\end{aligned}
$$

where the constants $C_{11}$ and $C_{12}$ depend on the coefficients $\alpha, g, M, \mu$ and the norms $\|f\|_{L^{2}\left(\Omega ; L^{2}\left(0, T ; \mathbb{H}_{0}^{1}\right)\right)}$, $\left\|w^{0}\right\|_{L^{4}\left(\Omega ; L^{4}\left(0, T ; \mathbb{H}_{0}^{1}\right)\right)},\left\|u_{0}\right\|_{L^{2}\left(\Omega ; \mathbb{H}_{0}^{1}\right)},\left\|\hat{z}_{0}\right\|_{L^{2}\left(\Omega ; \mathbb{H}_{0}^{1}\right)}$ and $T$.

Proof. Define

$$
\tau_{N}:=\inf \left\{t \geq 0:\left\|u^{n}(t)\right\|_{\mathbb{H}_{0}^{1}}^{2}+\left\|\hat{z}^{n}(t)\right\|_{\mathbb{H}_{0}^{1}}^{2}+\frac{3 \alpha}{4} \int_{0}^{t}\left\|\Delta u^{n}(s)\right\|_{\mathbb{L}^{2}}^{2} d s>N\right\}
$$

as the stopping time. Applying Itô's lemma to the process $\left\|\nabla u^{n}(t)\right\|_{\mathbb{L}^{2}}^{2}$

$$
\begin{aligned}
& \left\|u^{n}\left(t \wedge \tau_{N}\right)\right\|_{\mathbb{H}_{0}^{1}}^{2}+2 \alpha \int_{0}^{t \wedge \tau_{N}}\left\|\Delta u^{n}(s)\right\|_{\mathbb{L}^{2}}^{2} d s+2 \int_{0}^{t \wedge \tau_{N}}\left(\nabla B\left(u^{n}(s)\right), \nabla u^{n}(s)\right)_{\mathbb{L}^{2}} d s \\
& +2 \int_{0}^{t \wedge \tau_{N}}\left\langle\nabla\left(g \nabla \hat{z}^{n}(s)\right), \nabla u^{n}(s)\right\rangle d s=2 \int_{0}^{t \wedge \tau_{N}}\left(\nabla f, \nabla u^{n}(s)\right)_{\mathbb{L}^{2}} d s \\
& +2 \int_{0}^{t \wedge \tau_{N}}\left(\nabla \sigma^{n}\left(s, u^{n}(s)\right) d W^{n}(s), \nabla u^{n}(s)\right)_{\mathbb{L}^{2}} \\
& +\int_{0}^{t \wedge \tau_{N}}\left\|\nabla \sigma^{n}\left(s, u^{n}(s)\right)\right\|_{L_{Q}\left(\mathbb{L}^{2}, \mathbb{L}^{2}\right)}^{2} d s \\
& +\int_{0}^{t \wedge \tau_{N}} \int_{Z}\left\|\nabla H^{n}\left(u^{n}(s-), z\right)\right\|_{\mathbb{L}^{2}}^{2} N(d s, d z)+\left\|u^{n}(0)\right\|_{\mathbb{H}_{0}^{1}}^{2} \\
& +2 \int_{0}^{t \wedge \tau_{N}} \int_{Z}\left(\nabla H^{n}\left(u^{n}(s-), z\right), \nabla u^{n}(s-)\right)_{\mathbb{L}^{2}} \tilde{N}(d s, d z) .
\end{aligned}
$$

Using divergence theorem we obtain

$$
\begin{aligned}
& \left\|u^{n}\left(t \wedge \tau_{N}\right)\right\|_{\mathbb{H}_{0}^{1}}^{2}+2 \alpha \int_{0}^{t \wedge \tau_{N}}\left\|\triangle u^{n}(s)\right\|_{\mathbb{L}^{2}}^{2} d s \\
& =\left\|u^{n}(0)\right\|_{\mathbb{H}_{0}^{1}}^{2}+2 \int_{0}^{t \wedge \tau_{N}}\left(B\left(u^{n}(s)\right), \Delta u^{n}(s)\right)_{\mathbb{L}^{2}} d s \\
& \quad+2 \int_{0}^{t \wedge \tau_{N}}\left(g \nabla \hat{z}^{n}(s), \triangle u^{n}(s)\right)_{\mathbb{L}^{2}} d s+2 \int_{0}^{t \wedge \tau_{N}}\left(\nabla f, \nabla u^{n}(s)\right)_{\mathbb{L}^{2}} d s \\
& \quad+2 \int_{0}^{t \wedge \tau_{N}}\left(\nabla \sigma^{n}\left(s, u^{n}(s)\right) d W^{n}(s), \nabla u^{n}(s)\right)_{\mathbb{L}^{2}} \\
& \quad+\int_{0}^{t \wedge \tau_{N}}\left\|\nabla \sigma^{n}\left(s, u^{n}(s)\right)\right\|_{L_{Q}\left(\mathbb{L}^{2}, \mathbb{L}^{2}\right)}^{2} d s
\end{aligned}
$$




$$
\begin{aligned}
& +\int_{0}^{t \wedge \tau_{N}} \int_{Z}\left\|\nabla H^{n}\left(u^{n}(s-), z\right)\right\|_{\mathbb{L}^{2}}^{2} N(d s, d z) \\
& +2 \int_{0}^{t \wedge \tau_{N}} \int_{Z}\left(\nabla H^{n}\left(u^{n}(s-), z\right), \nabla u^{n}(s-)\right)_{\mathbb{L}^{2}} \tilde{N}(d s, d z) .
\end{aligned}
$$

Let $\left\{e_{j}\right\}_{j=1}^{\infty}$ be an orthonormal basis in $\mathbb{L}^{2}(\mathcal{O})$ such that $Q e_{j}=\lambda_{j} e_{j}$ for all $j=1,2, \cdots$, where $\lambda_{j}$ are the eigenvalues of $Q$. Then by (3.15), we observe that

$$
\begin{aligned}
& \left\|\nabla \sigma^{n}\left(t, u^{n}(t)\right)\right\|_{L_{Q}\left(\mathbb{L}^{2}, \mathbb{L}^{2}\right)}^{2}=\sum_{j=1}^{\infty} \lambda_{j}\left\|\nabla \sigma^{n}\left(t, u^{n}(t)\right) e_{j}\right\|_{\mathbb{L}^{2}}^{2} \\
& =\sum_{j=1}^{\infty} \lambda_{j}\left\|\sigma^{n}\left(t, u^{n}(t)\right) e_{j}\right\|_{\mathbb{H}_{0}^{1}}^{2}=\left\|\sigma^{n}\left(t, u^{n}(t)\right)\right\|_{L_{Q}\left(\mathbb{L}^{2}, \mathbb{H}_{0}^{1}\right)}^{2} .
\end{aligned}
$$

Using equation (3.11), Young's and Minkowskii inequalities, we obtain

$$
\begin{aligned}
2\left(B\left(u^{n}\right), \triangle u^{n}\right)_{\mathbb{L}^{2}} & \leq \frac{2}{\alpha}\left\|B\left(u^{n}\right)\right\|_{\mathbb{L}^{2}}^{2}+\frac{\alpha}{2}\left\|\Delta u^{n}(t)\right\|_{\mathbb{L}^{2}}^{2} \\
& \leq \frac{16}{\alpha}\left(\left\|u^{n}(t)\right\|_{\mathbb{L}^{4}}^{4}+\left\|w^{0}(t)\right\|_{\mathbb{L}^{4}}^{4}\right)+\frac{\alpha}{2}\left\|\triangle u^{n}(t)\right\|_{\mathbb{L}^{2}}^{2} .
\end{aligned}
$$

Using Young's inequality

$$
2\left|g\left(\nabla \hat{z}^{n}(t), \triangle u^{n}(t)\right)_{\mathbb{L}^{2}}\right| \leq \frac{2 g^{2}}{\alpha}\left\|\hat{z}^{n}(t)\right\|_{\mathbb{H}_{0}^{1}}^{2}+\frac{\alpha}{2}\left\|\triangle u^{n}(t)\right\|_{\mathbb{L}^{2}}^{2},
$$

and

$$
2\left(\nabla f, \nabla u^{n}(t)\right)_{\mathbb{L}^{2}} \leq\|f(t)\|_{\mathbb{H}_{0}^{1}}^{2}+\left\|u^{n}(t)\right\|_{\mathbb{H}_{0}^{1}}^{2}
$$

Hence using the above estimates in A.4

$$
\begin{aligned}
& \left\|u^{n}\left(t \wedge \tau_{N}\right)\right\|_{\mathbb{H}_{0}^{1}}^{2}+\alpha \int_{0}^{t \wedge \tau_{N}}\left\|\Delta u^{n}(s)\right\|_{\mathbb{L}^{2}}^{2} d s \\
& \leq \int_{0}^{t \wedge \tau_{N}}\left(\left\|u^{n}(s)\right\|_{\mathbb{H}_{0}^{1}}^{2}+\frac{16}{\alpha}\left\|u^{n}(s)\right\|_{\mathbb{L}^{4}}^{4}+\frac{2 g^{2}}{\alpha}\left\|\hat{z}^{n}(s)\right\|_{\mathbb{H}_{0}^{1}}^{2}+\|f(s)\|_{\mathbb{H}_{0}^{1}}^{2}\right. \\
& \left.\quad+\frac{16}{\alpha}\left\|w^{0}(s)\right\|_{\mathbb{L}^{4}}^{4}\right) d s+\int_{0}^{t \wedge \tau_{N}}\left\|\sigma^{n}\left(s, u^{n}(s)\right)\right\|_{L_{Q}\left(\mathbb{L}^{2}, \mathbb{H}_{0}^{1}\right)}^{2} d s \\
& \quad+\int_{0}^{t \wedge \tau_{N}} \int_{Z}\left\|H^{n}\left(u^{n}(s-), z\right)\right\|_{\mathbb{H}_{0}^{1}}^{2} N(d s, d z) \\
& \quad+2 \int_{0}^{t \wedge \tau_{N}}\left(\nabla \sigma^{n}\left(s, u^{n}(s)\right) d W^{n}(s), \nabla u^{n}(s)\right)_{\mathbb{L}^{2}} \\
& \quad+2 \int_{0}^{t \wedge \tau_{N}} \int_{Z}\left(\nabla H^{n}\left(u^{n}(s-), z\right), \nabla u^{n}(s-)\right)_{\mathbb{L}^{2}} \tilde{N}(d s, d z)+\|u(0)\|_{\mathbb{H}_{0}^{1}}^{2} .
\end{aligned}
$$

Now assuming an additional boundary condition that the gradient of the displacement of the free surface with respect to the ocean bottom is zero on the boundary of the domain (i.e. $\nabla \hat{z}=0$ on $[0, T] \times \partial \mathcal{O})$, we have from $(4.3)$

$$
\begin{aligned}
\left\|\nabla \hat{z}^{n}\left(t \wedge \tau_{N}\right)\right\|_{\mathbb{L}^{2}}^{2}= & -2 \int_{0}^{t \wedge \tau_{N}}\left(\nabla\left(\operatorname{Div}\left(h u^{n}(s)\right)\right), \nabla \hat{z}^{n}(s)\right)_{\mathbb{L}^{2}} d s+\left\|\nabla \hat{z}^{n}(0)\right\|_{\mathbb{L}^{2}}^{2} \\
= & -2 \int_{0}^{t \wedge \tau_{N}}\left(\nabla\left(h \operatorname{Divu}^{n}(s)\right)+\nabla\left(\nabla h \cdot u^{n}(s)\right), \nabla \hat{z}^{n}(s)\right)_{\mathbb{L}^{2}} d s \\
& +\left\|\nabla \hat{z}^{n}(0)\right\|_{\mathbb{L}^{2}}^{2}:=\int_{0}^{t \wedge \tau_{N}}\left(I_{1}+I_{2}\right) d s+\left\|\nabla \hat{z}^{n}(0)\right\|_{\mathbb{L}^{2}}^{2} .
\end{aligned}
$$

Now by use of product rule of gradient, assumption A.5, Minkowskii, Hölder's and Young's inequalities, we have

$$
\left|I_{1}\right| \leq 2\left\|\operatorname{Divu}^{n}(t) \nabla h+h \nabla\left(\operatorname{Divu}^{n}(t)\right)\right\|_{\mathbb{L}^{2}}\left\|\nabla \hat{z}^{n}(t)\right\|_{\mathbb{L}^{2}}
$$




$$
\begin{aligned}
& \leq 2\left(\|\nabla h\|_{\mathbb{L}^{\infty}}\left\|\operatorname{Divu}^{n}(t)\right\|_{L^{2}}+\|h\|_{L^{\infty}}\left\|\nabla\left(\operatorname{Divu}^{n}(t)\right)\right\|_{\mathbb{L}^{2}}\right)\left\|\nabla \hat{z}^{n}(t)\right\|_{\mathbb{L}^{2}} \\
& \leq 2\left(M\left\|u^{n}(t)\right\|_{\mathbb{H}_{0}^{1}}+\mu\left\|\nabla\left(D_{i v u}(t)\right)\right\|_{\mathbb{L}^{2}}\right)\left\|\nabla \hat{z}^{n}(t)\right\|_{\mathbb{L}^{2}} \\
& \leq 2\left(M\left\|u^{n}(t)\right\|_{\mathbb{H}_{0}^{1}}+\mu\left\|\triangle u^{n}(t)+\operatorname{curl} \operatorname{curlu}^{n}(t)\right\|_{\mathbb{L}^{2}}\right)\left\|\nabla \hat{z}^{n}(t)\right\|_{\mathbb{L}^{2}} \\
& =2\left(M\left\|u^{n}(t)\right\|_{\mathbb{H}_{0}^{1}}+\mu\left\|\triangle u^{n}(t)\right\|_{\mathbb{L}^{2}}\right)\left\|\nabla \hat{z}^{n}(t)\right\|_{\mathbb{L}^{2}} \\
& \leq M\left(\left\|u^{n}(t)\right\|_{\mathbb{H}_{0}^{1}}^{2}+\left\|\hat{z}^{n}(t)\right\|_{\mathbb{H}_{0}^{1}}^{2}\right)+\frac{\alpha}{4}\left\|\Delta u^{n}(t)\right\|_{\mathbb{L}^{2}}^{2}+\frac{2 \mu^{2}}{\alpha}\left\|\hat{z}^{n}(t)\right\|_{\mathbb{H}_{0}^{1}}^{2} \\
& \leq\left(M+\frac{2 \mu^{2}}{\alpha}\right)\left(\left\|u^{n}(t)\right\|_{\mathbb{H}_{0}^{1}}^{2}+\left\|\hat{z}^{n}(t)\right\|_{\mathbb{H}_{0}^{1}}^{2}\right)+\frac{\alpha}{4}\left\|\triangle u^{n}(t)\right\|_{\mathbb{L}^{2}}^{2} .
\end{aligned}
$$

By use of classical tame estimates for the product in Sobolev spaces (see, for instance, Corollary 2.4.1 of [14]), we have

$$
\begin{aligned}
& \left|I_{2}\right| \leq 2\left\|\nabla\left(\nabla h \cdot u^{n}(t)\right)\right\|_{\mathbb{L}^{2}}\left\|\nabla \hat{z}^{n}(t)\right\|_{\mathbb{L}^{2}} \\
& \leq 2 c\left(\|\nabla h\|_{\mathbb{L}^{\infty}}\left\|\nabla u^{n}(t)\right\|_{\mathbb{L}^{2}}+\|\nabla(\nabla h)\|_{\mathbb{L}^{\infty}}\left\|u^{n}(t)\right\|_{\mathbb{L}^{2}}\right)\left\|\nabla \hat{z}^{n}(t)\right\|_{\mathbb{L}^{2}} \\
& \leq c\left(M+N c_{P}\right)\left(\left\|u^{n}(t)\right\|_{\mathbb{H}_{0}^{1}}^{2}+\left\|\hat{z}^{n}(t)\right\|_{\mathbb{H}_{0}^{1}}^{2}\right),
\end{aligned}
$$

where $N:=\max _{x \in \mathcal{O}}|\nabla(\nabla h(x))|$ (as $h \in C_{b}^{2}(\mathcal{O})$ by assumption A.4), and $c_{P}$ is the Poincaré constant.

Hence from the estimates of $I_{1}$ and $I_{2}$, we have from (A.9),

$$
\begin{aligned}
\left\|\hat{z}^{n}\left(t \wedge \tau_{N}\right)\right\|_{\mathbb{H}_{0}^{1}}^{2} \leq & \|\hat{z}(0)\|_{\mathbb{H}_{0}^{1}}^{2}+C \int_{0}^{t \wedge \tau_{N}}\left(\left\|u^{n}(s)\right\|_{\mathbb{H}_{0}^{1}}^{2}+\left\|\hat{z}^{n}(s)\right\|_{\mathbb{H}_{0}^{1}}^{2}\right) d s \\
& +\frac{\alpha}{4} \int_{0}^{t \wedge \tau_{N}}\left\|\triangle u^{n}(s)\right\|_{\mathbb{L}^{2}}^{2} d s,
\end{aligned}
$$

where $C:=M+\frac{2 \mu^{2}}{\alpha}+c\left(M+N c_{P}\right)$.

Adding equations (A.8) and (A.10), we obtain

$$
\begin{aligned}
& \left\|u^{n}\left(t \wedge \tau_{N}\right)\right\|_{\mathbb{H}_{0}^{1}}^{2}+\left\|\hat{z}^{n}\left(t \wedge \tau_{N}\right)\right\|_{\mathbb{H}_{0}^{1}}^{2}+\frac{3 \alpha}{4} \int_{0}^{t \wedge \tau_{N}}\left\|\triangle u^{n}(s)\right\|_{\mathbb{L}^{2}}^{2} d s \\
& \leq K_{1}\left[\int_{0}^{t \wedge \tau_{N}}\left(\left\|u^{n}(s)\right\|_{\mathbb{H}_{0}^{1}}^{2}+\left\|\hat{z}^{n}(s)\right\|_{\mathbb{H}_{0}^{1}}^{2}\right) d s\right]+\frac{16}{\alpha} \int_{0}^{t \wedge \tau_{N}}\left\|u^{n}(s)\right\|_{\mathbb{L}^{4}}^{4} d s \\
& \quad+\int_{0}^{t \wedge \tau_{N}}\left(\|f(s)\|_{\mathbb{H}_{0}^{1}}^{2}+\frac{16}{\alpha}\left\|w^{0}(s)\right\|_{\mathbb{L}^{4}}^{4}\right) d s+\left\|u_{0}^{n}\right\|_{\mathbb{H}_{0}^{1}}^{2}+\left\|\hat{z}_{0}^{n}\right\|_{\mathbb{H}_{0}^{1}}^{2} \\
& \quad+K\left(t \wedge \tau_{N}\right),
\end{aligned}
$$

where $K_{1}:=\max \left\{1+C, 2 g^{2} / \alpha+C\right\}$.

Following the same steps as in Proposition 4.3, we integrate over $0 \leq t \leq T \wedge \tau_{N}$ and take expectation on both sides and employ the facts that $(i)$ stochastic integrals appearing in our calculations are martingales with zero average, $(i i)$ expectation of quadratic variation process and that of Meyer process are same and (iii) assumption A.2, to obtain

$$
\begin{aligned}
\mathbb{E} & {\left[\left\|u^{n}\left(t \wedge \tau_{N}\right)\right\|_{\mathbb{H}_{0}^{1}}^{2}+\left\|\hat{z}^{n}\left(t \wedge \tau_{N}\right)\right\|_{\mathbb{H}_{0}^{1}}^{2}\right]+\frac{3 \alpha}{4} \mathbb{E} \int_{0}^{t \wedge \tau_{N}}\left\|\Delta u^{n}(s)\right\|_{\mathbb{L}^{2}}^{2} d s } \\
\leq & K_{1} \mathbb{E}\left[\int_{0}^{t \wedge \tau_{N}}\left(\left\|u^{n}(s)\right\|_{\mathbb{H}_{0}^{1}}^{2}+\left\|\hat{z}^{n}(s)\right\|_{\mathbb{H}_{0}^{1}}^{2}\right) d s\right]+\frac{16}{\alpha} \mathbb{E} \int_{0}^{t \wedge \tau_{N}}\left\|u^{n}(s)\right\|_{\mathbb{L}^{4}}^{4} d s \\
& +\mathbb{E}\left[\int_{0}^{t \wedge \tau_{N}}\left(\|f(s)\|_{\mathbb{H}_{0}^{1}}^{2}+\frac{16}{\alpha}\left\|w^{0}(s)\right\|_{\mathbb{L}^{4}}^{4}\right) d s\right]+\mathbb{E}\left[\left\|u_{0}^{n}\right\|_{\mathbb{H}_{0}^{1}}^{2}+\left\|\hat{z}_{0}^{n}\right\|_{\mathbb{H}_{0}^{1}}^{2}\right] \\
& +K\left(t \wedge \tau_{N}\right),
\end{aligned}
$$

As before we observe that since $w^{0} \in L^{8}\left(\Omega ; L^{8}\left(0, T ; \mathbb{L}^{8}(\mathcal{O})\right)\right) \subset L^{4}\left(\Omega ; L^{4}(0, T\right.$;

$\left.\mathbb{L}^{4}(\mathcal{O})\right)$ ), by Proposition 4.4 for $p=4$, we have $\mathbb{E}\left[\int_{0}^{T}\left\|u^{n}(s)\right\|_{\mathbb{L}^{2}}^{2}\left\|u^{n}(s)\right\|_{\mathbb{H}_{0}^{1}}^{2} d s\right] \leq C_{1(4)}$, which essentially yields $\mathbb{E}\left[\int_{0}^{T}\left\|u^{n}(s)\right\|_{\mathbb{L}^{4}}^{4} d s\right] \leq C_{1(4)}$ (by the estimate (3.5)). 
Hence by applying Gronwall's lemma and taking limit as $N \rightarrow \infty$ we have the desired a-priori estimate (A.2).

To prove (A.3) one can proceed similarly but needs to take supremum over $\left[0, T \wedge \tau_{N}\right]$ over (A.11) before taking expectation and then suitably apply Burkholder-Davis-Gundy inequality.

Acknowledgements: Pooja Agarwal would like to thank Department of Science and Technology, Govt. of India, for the INSPIRE fellowship. Utpal Manna's work has been supported by the National Board of Higher Mathematics of Department of Atomic Energy, Govt. of India, under Grant No. NBHM/RP46/2013/Fresh/421. The authors would also like to thank Sakthivel Kumarasamy of Indian Institute of Space Science and Technology for his comments and pointing our attention to certain references. Finally, the authors would like to sincerely thank the anonymous referee for his/her valuable comments and criticisms which led to the improvement of this paper.

\section{REFERENCES}

[1] Adams, R. A. (1975). Sobolev Spaces, Academic Press, New York.

[2] Aldous, D. (1978). Stopping times and tightness; Ann. Probab., Vol. 6, pp. 335-340.

[3] Applebaum, D. (2009). Lévy Processes and Stochastic Calculus, Cambridge University Press, Second edition.

[4] Albeverio, S., Brzezniak, Z., And Wu., J.L. (2010). Existence of global solutions and invariant measures for stochastic differential equations driven by Poisson type noise with non-Lipschitz coefficients, Journal of Mathematical Analysis and Applications, Vol. 371, pp. 309-322.

[5] Aubin, J. P. and Ekeland, I. (1984). Applied Nonlinear Analysis, Wiley-Interscience, New York.

[6] Barbu, V. (1993). Analysis and Control of Nonlinear Infinite Dimensional Systems, Academic Press Inc., New York.

[7] Billingsley, P. (1969). Convergence of Probability Measures, Wiely, New York.

[8] Brzeźniak, Z., Hausenblas, E. and Razafimandimby, P. A. (2014). Stochastic reaction diffusion equation driven by jump processes; arXiv preprint arXiv:1010.5933.

[9] Brzeźniak, Z., Hausenblas, E. and Zhu, J. (2013). 2D stochastic Navier-Stokes equations driven by jump noise; Nonlinear Analysis, Vol. 79, pp. 122-139.

[10] Brzeźniak, Z. And Liu, W. And Zhu, J. (2014). Strong solutions for SPDE with locally monotone coefficients driven by Lévy noise; Nonlinear Anal. Real World Appl., Vol. 17, pp. 283-310.

[11] Brzeźniak, Z. and Motyl, E. (2013). Existence of a martingale solution of the stochastic Navier-Stokes equations in unbounded 2D and 3D domains; Journal of Differential Equations, pp. 1627-1685.

[12] Brzeźniak, Z., and Serrano, R. (2013). Optimal relaxed control of dissipative stochastic partial differential equations in Banach spaces, Siam J. Control Optim., Vol. 51(3), pp. 2664-2703.

[13] Castaing, C., Raynaud de Fitte, P., and Valadier, M. (2004). Young Measures on Topological Spaces: With Applications in Control Theory and Probability Theory, Math. Appl. 571, Kluwer Academic Publishers, Dordrecht, The Netherlands.

[14] Chemin, J.-Y. (1998). Perfect Incompressible Fluids, Oxford University Press, New York.

[15] Chow, P. L. (2007). Stochastic Partial Differential Equations, Chapman and Hall/CRC, New York.

[16] Chow, P.L. And Khasminskit, R.Z. (1997). Stationary solutions of nonlinear stochastic evolution equations; Stochastic Anal. Appl., Vol. 15 (5), pp 671-699.

[17] Da Prato, G. And ZabczyK, J. (1992). Stochastic Equations in Infinite Dimensions, Cambridge University Press.

[18] Dellacherie, C. and Meyer, P. A. (1975). Probabilitiés et Potential, Hermann, Paris.

[19] Flandoli, F., and Gatarek, D. (1995). Martingale and stationary solutions for stochastic Navier-Stokes equations; Probab. Theory Relat. Fields, Vol. 102, 367-391.

[20] Gawarecki, L. and Mandrekar, V. (2011). Stochastic Differential Equations in Infinite Dimensions with Applications to Stochastic Partial Differential Equations, Springer-Verlag, New York.

[21] Glatt-Holtz, N. and Ziane, M. (2009). Strong pathwise solutions of the stochastic Navier-Stokes system; Advances in Differential Equations, Vol. 14 (5/6), pp. 567-600.

[22] Ichikawa, A. (1986). Some inequalities for martingales and stochastic convolutions. Stochastic Anal. Appl. Vol. 4 (3), 329-339.

[23] JACOD, J. And MÉmin, J. (1981). Sur un type de convergence intermédiaire entre la convergence en loi et la convergence en probabilité; Séminaire de Probabilités XV 1979/80, pp. 529-546.

[24] Kesavan, S. (2004). Nonlinear Functional Analysis; A First Course, Hindustan Book Agency, New Delhi.

[25] Ladyzhenskaya, O. A. (1969). The Mathematical Theory of Viscous Incompressible Flow, Gordon and Breach, New York.

[26] Lions, J. L. (1969). Quelques méthodes de résolution des problèmes aux limites non linéaries, Dunod, Paris.

[27] Mandrekar, V. And Rüdiger, B. (2006). Lévy Noises and Stochastic Integrals on Banach Spaces. Stochastic Partial Differential Equations and Applications-VII, Lect. Notes Pure Appl. Math., Chapman \& Hall/CRC, Boca Raton, 7, 193-213. 
[28] Mandrekar, V. And Rüdiger, B. (2015). Stochastic Integration in Banach Spaces: Theory and Applications, Springer Cham Heidelberg New York.

[29] Manna, U. and Mohan, M. T. (2011). Shell model of turbulence perturbed by Lévy noise; Nonlinear Differential Equations and Applications NoDEA, Vol. 18(6), pp. 615-648.

[30] Manna, U., Mohan, M. T. and Sritharan, S. S. Stochastic Non-Resistive Magnetohydrodynamic System with Lévy Noise; preprint.

[31] Manna, U. and Menaldi, J. L. and Sritharan, S. S. (2008). Stochastic Analysis of Tidal Dynamics Equation; Infinite Dimensional Stochastic Analysis, Special Volume in honor of Professor HH. Kuo, Edited by A. Sengupta and P. Sundar, World Scientific Publishers.

[32] Marchuk, G. I. And Kagan, B. A. (1989). Dynamics of Ocean Tides, Kluwer Academic Publishers, Dordrecht/Boston/London.

[33] Marineldi, C. And Röckner M. (2015). On the Maximal Inequalities of Burkholder, Davis and Gundy, Expositiones Mathematicae, Article in Press.

[34] Métivier, M. (1988). Stochastic Partial Differential Equations in Infinite Dimensional Spaces, Scuola Normale Superiore, Pisa.

[35] Mikulevicius, R. And Rozovskit, B. L. (2005). Global $L^{2}$-solutions of stochastic Navier-Stokes equations; Ann. Probab., Vol. 33(1), pp. 137- 176.

[36] MoтүL, E. (2014). Stochastic hydrodynamic-type evolution equations driven by Lévy noise in 3D unbounded domains-Abstract framework and applications; Stochastic Processes and their Applications, Vol. 124, pp. 20522097.

[37] MотүL, E. (2013). Stochastic Navier-Stokes Equations Driven by Lévy noise in Unbounded 3D Domains; Potential Analysis, Vol. 38(3), pp. 863-912.

[38] MotyL, E. (2011). Martingale Solutions to the 2D and 3D Stochastic Navier-Stokes Equations Driven by the Compensated Poisson Random Measure; Preprint 13. Department of Mathematics and Computer Sciences, Lodz University.

[39] OndrejÁt, M. (2004). Uniqueness for stochastic evolution equations in Banach spaces, Dissertationes Mathematicae, 426, pp. 1-63.

[40] Peszat, S., And ZabczyK, J. (2007) Stochastic Partial Differential Equations with Lévy Noise, Encyclopedia of Mathematics and Its Applications 113, Cambridge University Press.

[41] Rüdiger, B. (2004). Stochastic integration with respect to compensated Poisson random measures on separable Banach spaces; Stochastics and Stochastics Reports, Vol. 76(3), pp.213-242.

[42] Rüdiger, B., And Ziglio, G. (2006). Itô Formula for Stochastic Integrals w.r.t. Compensated Poisson Random Measures on Separable Banach Spaces; Stochastics 78(6), pp. $377-410$.

[43] Sakthivel, K. and Sritharan, S. S. (2012). Martingale solutions for stochastic Navier-Stokes equations driven by Lévy noise; Evolution Equations and Control Theory, Vol. 1(2), pp. 355-392.

[44] Sritharan, S. S. (2000). Deterministic and Stochastic Control of Navier-Stokes Equation with Linear, Monotone and Hyperviscosities; Applied Mathematics and Optimization, Vol. 41(2), pp. 255-308.

[45] SRItharan, S. S. (1998). Optimal control of viscous flow, SIAM.

[46] Stroock, D. and Varadhan, S. R. S. (1979). Multidimensional Diffusion Processes, Springer-Verlag, New York.

[47] Suvinthra, M., Sritharan, S. S. and Balachandran, K (2015). Large deviations for stochastic tidal dynamics equation; Communications on Stochastic Analysis, Vol. 9(4), pp. 477-502.

[48] Temam, R. (1984). Navier-Stokes Equation: Theory and Numerical Analysis, AMS Chelsa Publishing.

[49] Vakhania, N.N. , Tarieladze, V.I. and Chobanyan, S.A. (1987). Probability distributions on Banach spaces, D. Reidel Publishing Company.

[50] Viот, M. (1976). Solution faibles d'equations aux derivees partielles stochastique nonlineaires. These, Universite Pierre et Marie Curie, Paris.

[51] Vishik, M. J. and Fursikov, A. V. (1988). Mathematical Problems of Statistical Hydromechanics, Kluwer Academic Publishers, Dordrecht.

[52] Watanabe, S. and Yamada, T. (1971). On the uniqueness of solutions of stochastic differential equations. II. J. Math. Kyoto Univ. 11, pp. 155-167.

Division of Applied Mathematics, Brown University, Providence, Rhode Island 02912, USA

E-mail address: Pooja_Agarwal@Brown.edu

Indian Institute of Science Education and Research Thiruvananthapuram, Thiruvananthapuram 695016, Kerala, India

E-mail address: manna.utpal@iisertvm.ac.in

Indian Institute of Science Education and Research Thiruvananthapuram, Thiruvananthapuram 695016, Kerala, India

E-mail address: debopriya13@iisertvm.ac.in 